\title{
The cosmological billiard attractor
}

\author{
J. Mark Heinzle ${ }^{1}$, Claes Uggla ${ }^{2}$, and Niklas Röhr ${ }^{2}$ \\ ${ }^{1}$ Gravitational Physics, Faculty of Physics, University of Vienna, A-1090 \\ Vienna, Austria \\ mark.heinzle@univie.ac.at \\ ${ }^{2}$ Department of Physics, University of Karlstad, S-651 88 Karlstad, Sweden \\ Claes.Uggla@kau.se
}

\begin{abstract}
This article is devoted to a study of the asymptotic dynamics of generic solutions of the Einstein vacuum equations toward a generic spacelike singularity. Starting from fundamental assumptions about the nature of generic spacelike singularities, we derive in a step-by-step manner the cosmological billiard conjecture: we show that the generic asymptotic dynamics of solutions is represented by (randomized) sequences of heteroclinic orbits on the "billiard attractor". Our analysis rests on two pillars: (i) a dynamical systems formulation based on the conformal Hubblenormalized orthonormal frame approach expressed in an Iwasawa frame; (ii) stochastic methods and the interplay between genericity and stochasticity. Our work generalizes and improves the level of rigor of previous work by Belinskii, Khalatnikov, and Lifshitz; furthermore, we establish that our approach and the Hamiltonian approach to "cosmological billiards", as elaborated by Damour, Hennaux, and Nicolai, can be viewed as yielding "dual" representations of the asymptotic dynamics.
\end{abstract}

e-print archive: http://lanl.arXiv.org/abs/gr-qc/0702141 


\section{Contents}

1 Introduction $\quad 298$

2 The Hamiltonian billiard approach 301

A brief review of the Hamiltonian approach to generic asymptotic dynamics. The asymptotic dynamical evolution is described as motion in "cosmological billiards".

3 The dynamical systems approach

An introduction to conformal Hubble-normalized variables and the associated dynamical systems approach to the Einstein field equations. We specialize to an Iwasawa decomposition of conformal orthonormal frames and give the definition of the state space associated with the Einstein equations in the chosen gauge.

\subsection{Conformal Hubble normalization}

\subsection{Iwasawa frame variables}

4 Asymptotic silence, locality, and the silent boundary

Discussion of the basic concepts and assumptions about generic spacelike singularities in the dynamical systems approach. Due to the spatial decoupling of the equations we can introduce a finite dimensional state space that is expected to capture the essential asymptotic dynamics - the so-called SH silent boundary.

\subsection{Asymptotic silence, and asymptotic locality}

\subsection{The silent boundary}

5 Kasner circle stability and the oscillatory subset

Discussion of important structures associated with the state space. We investigate the Kasner circle and the associated generalized Kasner solutions, and we reduce the SH silent boundary system to a system on an invariant subset: the oscillatory dynamical system. 
5.1 The Kasner circle, stable variables, and the oscillatory subset

5.2 The oscillatory dynamical system

6 Dynamics on the components of the oscillatory subset 323

Investigation of the state space and the dynamics of the oscillatory dynamical system. We analyze step-by-step the invariant subsets of the oscillatory system, which are associated with Bianchi types I, II, VI $I_{0}$, and VII . The orbits that turn out to be of prime importance for our purposes are "transitions", i.e., heteroclinic orbits that connect fixed points on the Kasner circle.

6.1 The silent Kasner subset (Bianchi type I subset)

6.2 The silent Bianchi type II subset

6.3 The silent Bianchi type $\mathrm{VI}_{0}$ and $\mathrm{VII}_{0}$ subsets

7 Sequences, eras, and phases

Definition and analysis of the fundamental objects for generic asymptotic dynamics of solutions: sequences of transitions. A sequence of transitions is defined to be an infinite concatenation of heteroclinic orbits on the oscillatory subset. Each (generic) sequence is naturally associated with a sequence of Kasner states that is determined by a recursive map, the so-called Kasner map. We investigate the basic properties of sequences of transitions and Kasner sequences; in particular we define the concepts of large and small curvature phases.

\subsection{Sequences of transitions and Kasner sequences}

7.2 Eras, large curvature phases, and small curvature phases

8 Stochastic analysis of Kasner sequences

Introduction to the stochastic aspects of our analysis. Kasner sequences possess a probabilistic interpretation, since they are associated with a probability density on a compact interval. We investigate Kasner sequences from this stochastic point of view. 
9 Growth

Investigation of growth rates. Along sequences of transitions several quantities associated with the state space variables exhibit well-defined growth rates. We give a detailed discussion of growth in terms of the growth function.

\subsection{Auxiliary differential equations and the growth function}

\subsection{Properties of the growth function}

10 Asymptotic shadowing

Discussion of a central issue: sequences of transitions act as the asymptotic limits of generic solutions of the Einstein equations with a spacelike singularity. However, not all sequences are admissible; by establishing the asymptotic suppression of certain variables, we restrict the set of possible "attractors" to billiard sequences. In this context stochastic methods are essential.

10.1 Shadowing - asymptotic sequences of orbits

$10.2 \quad R_{2} \rightarrow 0$ and $N_{2} \rightarrow 0$ as $\tau \rightarrow \infty$

10.3 Exclusion of $R_{2}$ and $N_{2}$

\subsection{Randomized Kasner sequences}

Definition and discussion of the billiard attractor. We reduce billiard sequences to attractor sequences, which are infinite concatenations of transitions of merely three types. We show that attractor sequences describe the asymptotic dynamics of generic solutions with a spacelike singularity. This section contains a brief summary of the main steps of the analysis.

11.1 The billiard subset

11.2 The asymptotic suppression of multiple transitions 
12 "Duality" of Hamiltonian and dynamical systems billiards

Discussion of the connection between the Hamiltonian approach and the dynamical systems approach. We show that the Hamiltonian cosmological billiard and the billiard attractor can be viewed as dual representations of generic asymptotic dynamics.

13 Models with symmetries in an Iwasawa frame

Specialization of our treatment to models with symmetries.

\section{1 $G_{1}$ models}

$13.2 \quad G_{2}$ models

13.3 Generic Bianchi type $\mathrm{VI}_{-1 / 9}$ models

14 Gauge considerations

Discussion of issues connected with gauge and analysis of gaugeinvariant quantities.

15 Concluding remarks

A brief recapitulation and discussion of some basic results.

We also give an outlook and make contact with related fields of research.

Acknowledgments

The paper concludes with a number of appendices that provide additional information on special issues. Some appendices contain rather technical computations that are omitted in the main text, while others are devoted to independent material: in particular, in Appendix B we derive the BKL generalized Kasner metric within the dynamical systems formulation, and in Appendix $H$ we establish asymptotic freezing, i.e., that certain variables converge to temporal constants in the asymptotic limit. 
A Iwasawa variables and useful equations 375

B Deriving the generalized Kasner line element of BKL

380

C Kasner solutions in a rotating frame

381

D Multiple transitions

383

E Behavior of an auxiliary quantity

387

F Convergence of sums

388

G Hitting intervals stochastically

393

H Asymptotic constants of the motion

395

H.1 Asymptotic freezing of $n_{i}$ and $\mathcal{P}_{i}$

396

H.2 Asymptotic freezing of $\pi_{\lambda}$

398

I AVTD singularities and the dynamical systems approach

References

404

\section{Introduction}

Remarkable developments took place in the sixties, seventies, and early eighties, that molded our understanding of singularities in general relativity (GR). On the one hand, the singularity theorems of Penrose and Hawking [1] proved the inevitability of spacetime singularities under rather general conditions. On the other hand, in a series of papers, Lifshitz, Khalatnikov and Belinskii, [2-4] and references therein, set out to give a description of the actual nature of generic singularities - henceforth we will refer to these authors and their work as BKL. These authors performed a heuristic analysis that eventually resulted in the claim that a generic singularity for the Einstein field equations with a perfect fluid with a radiation equation of state as the matter source is spacelike, local, vacuum 
dominated, and oscillatory. BKL obtained this picture by (i) using certain spatially homogeneous (SH) metrics, (ii) replacing constants with spatial functions, (iii) inserting the resulting expressions into Einstein's field equations and making a perturbative expansion, (iv) checking if this ad hoc procedure yielded a consistent result and thereby completing the basic viability test. Furthermore, BKL employed synchronous coordinates, i.e., Gaussian normal coordinates, such that the singularity occurred simultaneously. The BKL approach led to the conjecture that the time evolution of a generic solution in the vicinity of a generic singularity is schematically described by a sequence of generalized Kasner solutions (i.e., vacuum Bianchi type I solutions where constants are replaced with spatially dependent functions), where the transitions are "mediated" by generalized vacuum Bianchi type II solutions through the so-called Kasner map. In subsequent work, this map was shown to be associated with chaotic behavior $[5-7]$.

The BKL picture obtained further heuristic support from the Hamiltonian approach developed by Misner and Chitré [8-10], originally for asymptotic Bianchi type IX dynamics. In one variety of this approach the dynamics was described in terms of a free motion in an abstract flat Lorentzian (minisuper-) space surrounded by potential walls, or alternatively by means of a spatial projection leading to a free motion inside a potential well described by moving walls; for further developments of this picture, see [11] and [12, Chapter 10]. In another variety of the Hamiltonian approach, an intrinsic time variable was introduced, which led to a description of the asymptotic dynamics in terms of a projected "billiard" motion in a region of hyperbolic space bounded by infinitely high straight stationary walls, see e.g., [9]. This work on "cosmological billiards" was later generalized in order to deal with general inhomogeneous cases, which culminated in the recent work by Damour, Hennaux, and Nicolai, see [13, 14] and references therein. Apart from these studies, special inhomogeneous spacetimes with non-oscillatory singularities have also been investigated by means of Hamiltonian methods - notably by Moncrief, Isenberg, Berger, and collaborators, who also obtained numerical support for the general basic BKL picture, see [15] and [16] for a review and additional references.

Despite the ingenuity of the BKL and the Hamiltonian methods, there has been, unfortunately, little progress as regards a desired sharpening of the heuristic arguments in order to turn conjectures into rigorous mathematical statements. ${ }^{1}$ The requirement of mathematical rigor is met in a different

\footnotetext{
${ }^{1}$ Note, however, the successful rigorous treatment of special non-oscillatory cases in [17], which is partly based on Fuchsian methods.
} 
approach to cosmological singularities: the dynamical systems approach. During the last decade, based on dynamical systems formulations, there has been considerable progress in the $\mathrm{SH}$ case as regards theorems about dynamical behavior, largely in connection with the book "Dynamical Systems in Cosmology" [12]; notably, Ringström obtained the first mathematical theorems in the context of oscillatory behavior for Bianchi type VIII and, more substantially, type IX models $[18,19]$. In an attempt to extend the dynamical systems approach to the inhomogeneous context, Uggla et al. [20] introduced a dynamical systems formulation for the Einstein field equations without any symmetries - in the following, we will refer to this work as UEWE. The results were: a detailed description of the generic attractor; concisely formulated conjectures about the asymptotic dynamic behavior toward a generic spacelike singularity; a basis for a numerical investigation of generic singularities - following UEWE numerical results yielded additional support for the expected generic picture as well as the discovery of new phenomena and subsequent refinements [21-23].

The purpose of the present paper is 2-fold: first, we establish a link between the Hamiltonian picture as described by Damour and coworkers [13, 14] and the dynamical systems approach to inhomogeneous cosmologies as initiated in UEWE. In particular, we demonstrate that the "Hamiltonian billiards" and the corresponding dynamical systems description can be viewed as yielding dual representations of the generic asymptotic dynamics ("configuration space description" versus "momentum space description"). To avoid excessive clutter that would obscure the main ideas, we simplify our presentation by confining ourselves to the four-dimensional vacuum case. Inclusion of matter sources such as perfect fluids, see e.g., [20,26], and to more general cases like those considered by Damour and coworkers $[13,14]$ should - in principle - be straightforward.

The second and main purpose of this paper is to derive and give rigor to some of the key conjectures formulated in the BKL and Hamiltonian approach, starting from "first principles" connected with the full state space picture of our dynamical systems approach. This derivation does not constitute a mathematical proof of the conjectures; however, in many respects we go beyond what has been accomplished previously: we identify the "billiard attractor" as the attractor for the asymptotic dynamics toward a generic spacelike singularity; we give decay rates that describe the approach to the attractor; we show how asymptotic constants of the motion, which were obtained from the Hamiltonian billiard approach initially, arise. An important ingredient in our treatment is the use of stochastic methods: we emphasize the connection between genericity and stochasticity. The detailed statements and conjectures we obtain are accessible to numerical experiments and can be compared with results for special models. 
The present paper is to a large extent self-contained. First, we give a condensed review of the Hamiltonian approach of Damour and coworkers $[13,14]$ in Section 2. Second, we present the conformal Hubble-normalized orthonormal frame approach leading to the dynamical systems formulation in Sections 3 and 4 . Endowed with the basic techniques and the interrelation between the two approaches, we then derive the billiard attractor, which then serves as the starting point for further discussions, developments, and concluding remarks.

\section{The Hamiltonian billiard approach}

In [13], Damour, Henneaux, and Nicolai used a Hamiltonian approach to study the dynamics of the Einstein-dilaton $p$-form system in the neighborhood of a generic spacelike singularity. The authors described the asymptotic behavior of the fields by a "billiard" motion in a region of hyperbolic space bounded by straight "walls". The techniques used in [13] represent generalizations of methods that are due to Chitré and Misner [8-10], which have been applied and extended by many authors, see the references in [13]. In the following we give a brief review of the Hamiltonian "billiard approach" restricted to the four-dimensional vacuum case in GR; the presentation is based on [13], but the notation is tailored to our later purposes. In particular, for frame indices we use $\alpha, \beta, \ldots$, as in $[12,20]$, and for spatial coordinate indices we use $i, j, \ldots$, as in [13] and $[12,20]$.

In [13], the metric is written in $3+1$-form with vanishing shift vector,

$$
d s^{2}=-N^{2}\left(d x^{0}\right)^{2}+g_{i j} d x^{i} d x^{j} .
$$

Let $g^{i j}$ denote the inverse of the spatial three-metric $g_{i j}$ and $g=\operatorname{det} g_{i j}$. We introduce

$$
\mathcal{G}^{i j k l}:=g^{i(k} g^{l) j}-g^{i j} g^{k l} \quad \text { and } \quad \mathcal{G}_{i j k l}:=g_{i(k} g_{l) j}-\frac{1}{2} g_{i j} g_{k l} ;
$$

$\mathcal{G}^{i j k l}$ is the DeWitt metric [27] multiplied with $g^{-1 / 2}$ so that it becomes a tensor instead of a tensor density; $\mathcal{G}_{i j m n} \mathcal{G}^{m n k l}=\delta_{i j}{ }^{k l}=\delta_{(i}^{(k} \delta_{j)}^{l)}$.

The Lagrangian (density) associated with the reduced Einstein-Hilbert action reads

$$
\mathcal{L}=\frac{1}{4} \sqrt{g} N^{-1} \mathcal{G}^{i j k l} \dot{g}_{i j} \dot{g}_{k l}+\sqrt{g} N^{3} R,
$$

where a dot refers to the partial derivative w.r.t. $x^{0}$ and ${ }^{3} R$ denotes the spatial three-curvature associated with $g_{i j}$. To obtain the Hamiltonian $\mathcal{H}$ 
we introduce the conjugate momenta

$$
\pi^{i j}=\partial \mathcal{L} / \partial \dot{g}_{i j}=\frac{1}{2} \tilde{N}^{-1} \mathcal{G}^{i j k l} \dot{g}_{k l},
$$

where we define

$$
\tilde{N}=g^{-1 / 2} N
$$

This leads to

$$
\mathcal{L}=\pi^{i j} \dot{g}_{i j}-\mathcal{H}=\pi^{i j} \dot{g}_{i j}-\tilde{N}\left[\mathcal{G}_{i j k l} \pi^{i j} \pi^{k l}-g^{3} R\right] .
$$

Variation of $\mathcal{H}$ w.r.t. $\tilde{N}$ yields the Hamiltonian constraint $\mathcal{H}=0$, while the momentum constraints are introduced separately, see [13].

There exists a unique oriented orthonormal spatial coframe $\left\{\omega^{\alpha} \mid \alpha=\right.$ $1, \ldots, 3\}, \omega^{\alpha}=e^{\alpha}{ }_{i} d x^{i}$, such that $e^{\alpha}{ }_{i}=\sum_{\beta} \mathcal{D}^{\alpha}{ }_{\beta} \mathcal{N}^{\beta}{ }_{i}$, where $\mathcal{D}$ is a diagonal matrix, which we choose to express as $\mathcal{D}=\operatorname{diag}\left[\exp \left(-b^{1}\right)\right.$, exp $\left.\left(-b^{2}\right), \exp \left(-b^{3}\right)\right]$, and where $\mathcal{N}^{\alpha}{ }_{i}$ is a unit upper triangular matrix,

$$
\left(\mathcal{N}_{i}^{\alpha}\right)=\left(\begin{array}{ccc}
1 & \mathcal{N}^{1}{ }_{2} & \mathcal{N}^{1}{ }_{3} \\
0 & 1 & \mathcal{N}^{2}{ }_{3} \\
0 & 0 & 1
\end{array}\right)=\left(\begin{array}{ccc}
1 & n_{1} & n_{2} \\
0 & 1 & n_{3} \\
0 & 0 & 1
\end{array}\right) .
$$

This choice of frame leads to the so-called Iwasawa decomposition of the spatial metric $g_{i j}$ :

$$
g_{i j}=\sum_{\alpha} \exp \left(-2 b^{\alpha}\right) \mathcal{N}^{\alpha}{ }_{i} \mathcal{N}^{\alpha}{ }_{j}
$$

Existence and uniqueness of the frame $\left\{\omega^{\alpha}\right\}$ is associated with the theorem on the uniqueness of the QR decomposition in linear algebra; $\mathcal{N}^{\alpha}{ }_{i}$ can also be viewed as representing the Gram-Schmidt orthogonalization of the spatial coordinate coframe $\left\{d x^{i}\right\}$. The Iwasawa decomposition corresponds to a Cholesky decomposition of a symmetric matrix $A$ into a product $R^{\mathrm{T}} R$, where the diagonal elements of the triangular matrix $R$ are factored out and parameterized as $\exp \left(-b^{\alpha}\right)$.

To avoid confusion with the Greek frame indices $\alpha, \beta, \ldots$, we prefer to use $b$ as the kernel letter for the diagonal degrees of freedom instead of $\beta$, which was used by Damour et al. [13]. Note that the negative sign in the exponentials is in agreement with the conventions of [13], but contrary to the conventions of, e.g., $[11,12]$. For representations that are adapted to metric anisotropies, see Misner [8] and, e.g., [9,12]. Following the summation convention of [13], summation of pairs of coordinate indices $i, j, \ldots$ is 
understood, whereas sums over the frame indices $\alpha, \beta, \ldots$ are written out explicitly in this section.

The frame $\left\{e_{\alpha}\right\}$ that is dual to $\left\{\omega^{\alpha}\right\}$ is given by $e_{\alpha}=e_{\alpha}{ }^{i} \partial_{x^{i}}=\exp \left(b^{\alpha}\right)$ $\overline{\mathcal{N}}^{i}{ }_{\alpha} \partial_{x^{i}}$, where the matrix $\left(\overline{\mathcal{N}}^{i}{ }_{\alpha}\right)$ is the inverse of $\left(\mathcal{N}^{\alpha}{ }_{i}\right)$,

$$
\left(\overline{\mathcal{N}}^{i}{ }_{\alpha}\right)=\left(\begin{array}{ccc}
1 & \overline{\mathcal{N}}^{1}{ }_{2} & \overline{\mathcal{N}}^{1}{ }_{3} \\
0 & 1 & \overline{\mathcal{N}}^{2}{ }_{3} \\
0 & 0 & 1
\end{array}\right)=\left(\begin{array}{ccc}
1 & \bar{n}_{1} & \bar{n}_{2} \\
0 & 1 & \bar{n}_{3} \\
0 & 0 & 1
\end{array}\right)=\left(\begin{array}{ccc}
1 & -n_{1} & n_{1} n_{3}-n_{2} \\
0 & 1 & -n_{3} \\
0 & 0 & 1
\end{array}\right) .
$$

Expressing the Lagrangian (2.3) in the Iwasawa frame variables $b^{\alpha}$ and $\mathcal{N}^{\alpha}{ }_{i}$ yields

$$
\mathcal{L}=\sqrt{g} N^{-1}\left[\sum_{\alpha, \beta} \mathcal{G}_{\alpha \beta} \dot{b}^{\alpha} \dot{b}^{\beta}+\frac{1}{2} \sum_{\alpha<\beta} \exp \left(2\left(b^{\beta}-b^{\alpha}\right)\right)\left(\dot{\mathcal{N}}_{i}^{\alpha} \overline{\mathcal{N}}_{\beta}^{i}\right)^{2}\right]+\sqrt{g} N^{3} R,
$$

where we have introduced the reduced metric $\mathcal{G}_{\alpha \beta}$, which is associated with the diagonal degrees of freedom, i.e., " $b^{\alpha}$-space":

$$
\sum_{\alpha, \beta} \mathcal{G}_{\alpha \beta} v^{\alpha} w^{\beta}:=-\sum_{\gamma \neq \delta} v^{\gamma} w^{\delta}=\sum_{\alpha} v^{\alpha} w^{\alpha}-\left(\sum_{\alpha} v^{\alpha}\right)\left(\sum_{\beta} w^{\beta}\right)
$$

when " $b^{\alpha}$-space" is endowed with the metric $\mathcal{G}_{\alpha \beta}$, it is isometric to a $(2+1)$ dimensional Minkowski space, since the signature of $\mathcal{G}_{\alpha \beta}$ is $(-++)$. The inverse metric $\mathcal{G}^{\alpha \beta}$ of $\mathcal{G}_{\alpha \beta}$ is given by

$$
\sum_{\alpha, \beta} \mathcal{G}^{\alpha \beta} v_{\alpha} w_{\beta}=\sum_{\alpha, \beta} \delta^{\alpha \beta} v_{\alpha} w_{\beta}-\frac{1}{2} \sum_{\alpha, \beta} v_{\alpha} w_{\beta}=\sum_{\gamma} v_{\gamma} w_{\gamma}-\frac{1}{2}\left(\sum_{\alpha} v_{\alpha}\right)\left(\sum_{\beta} w_{\beta}\right) .
$$

The momenta $\pi_{\alpha}$ that are conjugate to $b^{\alpha}$ and the momenta $\mathcal{P}^{i}{ }_{\alpha}(i>\alpha)$ that are conjugate to $\mathcal{N}^{\alpha}{ }_{i}$ read

$$
\pi_{\alpha}=2 \tilde{N}^{-1} \sum_{\beta} \mathcal{G}_{\alpha \beta} \dot{b}^{\beta} ; \quad \mathcal{P}_{\alpha}^{i}=\tilde{N}^{-1} \sum_{\beta} e^{2\left(b^{\beta}-b^{\alpha}\right)} \dot{\mathcal{N}}_{j}^{\alpha} \overline{\mathcal{N}}^{j}{ }_{\beta} \overline{\mathcal{N}}^{i}{ }_{\beta} \quad(i>\alpha),
$$


while $\mathcal{P}_{\alpha}^{i}=0$ when $i \leq \alpha$; accordingly, $\mathcal{P}_{\alpha}^{i}$ can be viewed as a lower triangular matrix, whose components we for convenience call $\mathcal{P}_{i}$ :

$$
\left(\mathcal{P}^{i}{ }_{\alpha}\right)=\left(\begin{array}{ccc}
0 & 0 & 0 \\
\mathcal{P}^{2}{ }_{1} & 0 & 0 \\
\mathcal{P}^{3}{ }_{1} & \mathcal{P}_{2}^{3} & 0
\end{array}\right)=\left(\begin{array}{ccc}
0 & 0 & 0 \\
\mathcal{P}_{1} & 0 & 0 \\
\mathcal{P}_{2} & \mathcal{P}_{3} & 0
\end{array}\right)
$$

Inverting the relationships (2.7) yields

$$
\begin{aligned}
& \dot{b}^{\alpha}=\frac{1}{2} \tilde{N} \sum_{\beta} \mathcal{G}^{\alpha \beta} \pi_{\beta}, \quad \dot{n}_{2}=\tilde{N} e^{2\left(b^{1}-b^{2}\right)}\left(n_{3} \mathcal{P}_{1}+\left(e^{2\left(b^{2}-b^{3}\right)}+n_{3}^{2}\right) \mathcal{P}_{2}\right), \\
& \dot{n}_{1}=\tilde{N} e^{2\left(b^{1}-b^{2}\right)}\left(\mathcal{P}_{1}+n_{3} \mathcal{P}_{2}\right), \quad \dot{n}_{3}=\tilde{N} e^{2\left(b^{2}-b^{3}\right)} \mathcal{P}_{3},
\end{aligned}
$$

which leads to

$$
\begin{aligned}
\mathcal{L}= & \sum_{\alpha}\left(\pi_{\alpha} \dot{b}^{\alpha}+\mathcal{P}_{\alpha}^{i} \dot{\mathcal{N}}_{i}^{\alpha}\right) \\
& -\tilde{N}\left[\frac{1}{4} \sum_{\alpha, \beta} \mathcal{G}^{\alpha \beta} \pi_{\alpha} \pi_{\beta}+\frac{1}{2} \sum_{\alpha<\beta} e^{-2\left(b^{\beta}-b^{\alpha}\right)}\left(\mathcal{P}^{i}{ }_{\alpha} \mathcal{N}^{\beta}{ }_{i}\right)^{2}-g R\right],
\end{aligned}
$$

where the Hamiltonian $\mathcal{H}$ can be read off easily.

We assume that the spacetime with metric (2.1) possesses a spacelike singularity in the past; then, as argued in [13], $b^{\alpha}$ is expected to be timelike in the vicinity of this singularity, i.e., $\sum_{\alpha, \beta} \mathcal{G}_{\alpha \beta} b^{\alpha} b^{\beta}<0$. Based on these considerations, it is possible to replace the metric variables $b^{\alpha}$ by new variables: we introduce $\rho^{2}=-\sum_{\alpha, \beta} \mathcal{G}_{\alpha \beta} b^{\alpha} b^{\beta}$ and "orthogonal" angular variables, collectively denoted by $\gamma$, i.e., $\partial_{\gamma}$ is orthogonal to $\partial_{\rho}$. In these variables the line element associated with $\mathcal{G}_{\alpha \beta}$ can be written in the form

$$
d \sigma^{2}=\sum_{\alpha, \beta} \mathcal{G}_{\alpha \beta} d b^{\alpha} d b^{\beta}=-d \rho^{2}+\rho^{2} d \Omega_{h}^{2}
$$

where $d \Omega_{h}^{2}$ is the standard metric on hyperbolic space. Making a further variable change according to

$$
\lambda=\log \rho=\frac{1}{2} \log \left(-\sum_{\alpha, \beta} \mathcal{G}_{\alpha \beta} b^{\alpha} b^{\beta}\right)
$$


yields $d \sigma^{2}=\rho^{2}\left(-d \lambda^{2}+d \Omega_{h}^{2}\right)$ and thus in particular

$$
\sum_{\alpha, \beta} \mathcal{G}^{\alpha \beta} \pi_{\alpha} \pi_{\beta}=-\pi_{\rho}^{2}+\rho^{-2} \pi_{\gamma}^{2}=\rho^{-2}\left[-\pi_{\lambda}^{2}+\pi_{\gamma}^{2}\right]
$$

cf. (2.10).

Consequently, choosing the rescaled lapse according to $\tilde{N}=\rho^{2}$ leads to the Hamiltonian

$$
\mathcal{H}=\frac{1}{4}\left[-\pi_{\lambda}^{2}+\pi_{\gamma}^{2}\right]+\rho^{2} \sum_{A} c_{A} e^{-2 \rho w_{A}(\gamma)}
$$

here, the sum is over a number of terms of the same type: $c_{A}$ are functions of spatial derivatives of the metric, off-diagonal metric variables, and momenta; $w_{A}(\gamma)$ denote linear forms of the variables $\gamma^{\alpha}$, i.e., $w_{A}(\gamma)=\sum_{\alpha}\left(w_{A}\right)_{\alpha} \gamma^{\beta}$.

The Hamiltonian approach relies on the expectation that $\rho \rightarrow+\infty$ in the approach to the singularity. As a consequence of this assumption, each term $\rho^{2} \exp \left[-2 \rho w_{A}(\gamma)\right]$ becomes a sharp wall, i.e., an infinitely high potential which is described by an infinite step function $\Theta_{\infty}(x)$ that vanishes for $x<0$ and is infinite when $x \geq 0$. In the GR vacuum case there exist three "dominant" terms in the sum, which is the minimum number of terms required to define the "billiard table". The coefficients $c_{A}$ of the dominant terms are non-negative functions of the variables, but "generically" they are positive, see [13]. The three dominant terms $\rho^{2} c_{A} \exp \left[-2 \rho w_{A}(\gamma)\right]$ thus generate "dominant" walls $\Theta_{\infty}\left(-2 w_{A}(\gamma)\right)$. Only the dominant walls are assumed to be of importance for the generic asymptotic dynamics; the other, "subdominant", terms of (2.12), whose exponential $b^{\alpha}$-dependence can be obtained by multiplying dominant terms, are dropped accordingly. We thus obtain an asymptotic Hamiltonian of the form

$$
\mathcal{H}_{\infty}=\frac{1}{4}\left[-\pi_{\lambda}^{2}+\pi_{\gamma}^{2}\right]+\sum_{A=1}^{3} \Theta_{\infty}\left(-2 w_{A}(\gamma)\right),
$$

where the sum is over the three dominant terms.

This limiting Hamiltonian is believed to describe generic asymptotic dynamics. It is independent of $\mathcal{N}^{\alpha}{ }_{i}, \mathcal{P}^{i}{ }_{\alpha}$, and $\lambda$, which suggests that $\mathcal{P}^{i}{ }_{\alpha}$, $\mathcal{N}^{\alpha}{ }_{i}$, and $\pi_{\lambda}$ are asymptotic constants of the motion. The remaining nontrivial dynamics resides in hyperbolic space, i.e., in the variables $\gamma$. The asymptotic dynamics can be described via (2.13) as a geodesic motion in hyperbolic space, which is constrained by the existence of three sharp 
reflective walls, i.e., by a "billiard motion". The asymptotic dynamics is thus given by a "cosmological billiard", see figure 8(a).

\section{The dynamical systems approach}

\subsection{Conformal Hubble normalization}

Physics is a science of scales: in many problems there exists a variable scale of particular importance, which can be factored out of the equations in order to obtain a simpler mathematical description. In GR there exists a single dimensional unit which is chosen to be "length" (or, equivalently, "time"). The geometrical way of factoring out a scale in GR is by means of a conformal transformation: choose a conformal factor that depends on the variable scale so that the conformal factor carries the dimension and the conformal metric becomes dimensionless. Then, for dimensional reasons, the Einstein field equations split into decoupled equations for the conformal factor and a coupled system of dimensionless equations for quantities associated with the dimensionless conformal metric. ${ }^{2}$ This reduced dimensionless system carries the essential information about the problem, since one can solve for the decoupled equations for the conformal factor once it has been understood, and thus recover the physical metric.

The problem of generic spacelike singularities and the dynamics of solutions in a neighborhood thereof is one example where one has a variable scale: it is provided by the affine parameter of inextendible causal geodesics, or the expansion along such geodesics. Here we consider non-rotating timelike congruences that may or may not be geodesic; the latter is of no particular relevance, since the generic behavior toward a spacelike singularity is similar, as will be discussed later. One equation plays an essential role in the singularity theorems - the Raychaudhuri equation for the expansion. This makes a suitable function of the expansion an excellent candidate for a conformal factor. By factoring out the expansion, which blows up toward the singularity, we will obtain a system of regular dimensionless equations. ${ }^{3}$ Let us begin by introducing the basic set-up and nomenclature:

\footnotetext{
${ }^{2}$ Logarithmic derivatives of the conformal factor occur in the equations; however, one of the evolution equations (which is the Raychaudhuri equation in the present Hubblenormalized case) can be used to algebraically solve for the logarithmic time derivative, and the constraints can be used to generically solve for the logarithmic spatial derivatives.

${ }^{3}$ For historical reasons we will choose the Hubble variable instead of the expansion, but since they are proportional to each other, this makes no essential difference.
} 
Consider a four-dimensional Lorentzian manifold (the "physical spacetime") with a metric $\mathbf{g}$ of signature $(-+++)$ that satisfies the Einstein vacuum equations. By convention we set $c=1$ and $8 \pi G=1$, which leaves "length" (or, equivalently, "time") as the single remaining unit. Let us consider a timelike non-rotating congruence in the physical spacetime whose tangential vector field we denote by $\mathbf{u}$. We choose an orthonormal frame $\mathbf{e}_{a}$ $(a=0, \ldots, 3)$ such that $\mathbf{e}_{0}=\mathbf{u}$, and introduce an orthonormal coframe dual to $\mathbf{e}_{a}$ which we denote by $\boldsymbol{\omega}^{a}$, i.e., $\boldsymbol{\omega}^{a}\left(\mathbf{e}_{b}\right)=\delta_{b}^{a}$. Our choice of coordinates is that of a coordinate $3+1$ decomposition associated with $\mathbf{u}$, where we set the shift vector to zero and let $\partial_{x^{0}}=N \mathbf{u}$, so that the flow lines of $\mathbf{u}$ are the timelines. Accordingly,

$$
\mathbf{e}_{0}=N^{-1} \partial_{x^{0}}, \quad \mathbf{e}_{\alpha}=e_{\alpha}{ }^{i} \partial_{x^{i}} \quad \text { and } \quad \boldsymbol{\omega}^{0}=N d x^{0}, \quad \boldsymbol{\omega}^{\alpha}=e_{i}^{\alpha} d x^{i}
$$

with $\alpha=1,2,3$ and $i=1,2,3$. The metric thus reads

$$
\mathbf{g}=\eta_{a b} \boldsymbol{\omega}^{a} \boldsymbol{\omega}^{b}=-N^{2}\left(d x^{0}\right)^{2}+\delta_{\alpha \beta} e_{i}^{\alpha} e^{\beta}{ }_{j} d x^{i} d x^{j},
$$

where $\eta_{a b}=\operatorname{diag}(-1,1,1,1)$. The Hubble scalar of the vector field $\mathbf{u}=\mathbf{e}_{0}$ is given by $H=\frac{1}{3} \nabla_{a} \mathbf{u}^{a}$ where $\nabla$ denotes the covariant derivative associated with $\mathbf{g}$, and hence $H$ is related to the expansion $\theta$ of $\mathbf{u}$ by $H=\frac{1}{3} \theta$. Note that $H^{-1}$ carries dimension "length" (see $[24,28]$ and compare with discussions about FRW models where this is a frequently used fact).

The natural way of keeping track of dimensions in a parameterized theory like GR is by means of using rigid measuring rods that carry the dimension, which corresponds to assigning a dimension ("length") to the orthonormal one-forms. To obtain Hubble-normalized variables and associated Hubblenormalized equations, we factor out the Hubble scale out of the frame vectors and dual one-forms, i.e., we go over to a conformal Hubble-normalized orthonormal frame $\boldsymbol{\partial}_{a}$ and its dual coframe $\boldsymbol{\Omega}^{a}$ :

$$
\partial_{a}=\frac{\mathbf{e}_{a}}{H}, \quad \boldsymbol{\Omega}^{a}=H \boldsymbol{\omega}^{a} .
$$

The dimensionless "unphysical metric" $\mathbf{G}$ that is associated with the conformal frame is defined as $\mathbf{G}=\eta_{a b} \boldsymbol{\Omega}^{a} \boldsymbol{\Omega}^{b}$; it is related to $\mathbf{g}$ through a conformal rescaling:

$$
\mathbf{G}=\eta_{a b} \boldsymbol{\Omega}^{a} \boldsymbol{\Omega}^{b}=H^{2} \eta_{a b} \boldsymbol{\omega}^{a} \boldsymbol{\omega}^{b}=H^{2} \mathbf{g} .
$$


The conformal Hubble-normalized orthonormal frame satisfies

$$
\partial_{0}=\mathcal{N}^{-1} \partial_{x^{0}}, \quad \partial_{\alpha}=E_{\alpha}{ }^{i} \partial_{x^{i}} \quad \text { and } \quad \boldsymbol{\Omega}^{0}=\mathcal{N} d x^{0}, \quad \boldsymbol{\Omega}^{\alpha}=E_{i}^{\alpha} d x^{i},
$$

where $\mathcal{N}$ is the conformal lapse, $E_{\alpha}{ }^{i}$ are the conformal spatial frame vector components, and $E_{i}^{\alpha}$ the associated dual vector components, i.e.,

$$
\mathcal{N}=H N, \quad E_{\alpha}{ }^{i}=\frac{e_{\alpha}{ }^{i}}{H}, \quad E_{i}^{\alpha}=H e_{i}^{\alpha}
$$

Since $\boldsymbol{\Omega}^{\alpha}$ is dual to $\boldsymbol{\partial}_{\alpha}$, i.e., $\boldsymbol{\Omega}^{\alpha}\left(\boldsymbol{\partial}_{\beta}\right)=\delta_{\beta}^{\alpha}$, the same is true for the components $E_{\alpha}{ }^{i}$ and $E_{i}^{\alpha}$, i.e., $E_{i}^{\alpha} E_{\beta}{ }^{i}=\delta_{\beta}^{\alpha}$. The unphysical metric $\mathbf{G}$ can be written

$$
\mathbf{G}=\eta_{a b} \boldsymbol{\Omega}^{a} \boldsymbol{\Omega}^{b}=-\mathcal{N}^{2}\left(d x^{0}\right)^{2}+\delta_{\alpha \beta} E_{i}^{\alpha} E^{\beta}{ }_{j} d x^{i} d x^{j} .
$$

For further details and discussions see [24].

The starting point for the conformal Hubble-normalized orthonormal frame approach to the Einstein equations is the decomposition of the commutators of $\boldsymbol{\partial}_{a}$,

$$
\begin{aligned}
{\left[\boldsymbol{\partial}_{0}, \boldsymbol{\partial}_{\alpha}\right] } & =\dot{U}_{\alpha} \boldsymbol{\partial}_{0}+F_{\alpha}{ }^{\beta} \boldsymbol{\partial}_{\beta}, \quad F_{\alpha}{ }^{\beta}=q \delta_{\alpha}{ }^{\beta}-\Sigma_{\alpha}{ }^{\beta}-\epsilon_{\alpha}{ }^{\beta}{ }_{\gamma} R^{\gamma}, \\
{\left[\boldsymbol{\partial}_{\alpha}, \boldsymbol{\partial}_{\beta}\right] } & =\left(2 A_{[\alpha} \delta_{\beta]}{ }^{\gamma}+\epsilon_{\alpha \beta \delta} N^{\delta \gamma}\right) \boldsymbol{\partial}_{\gamma},
\end{aligned}
$$

which can also be written in the alternative form

$$
\begin{aligned}
& 0=\left(\boldsymbol{\partial}_{\alpha}+\dot{U}_{\alpha}\right) \boldsymbol{\partial}_{0}-\left(\delta_{\alpha}{ }^{\beta} \boldsymbol{\partial}_{0}-F_{\alpha}{ }^{\beta}\right) \boldsymbol{\partial}_{\beta}, \quad F_{\alpha}{ }^{\beta}=q \delta_{\alpha}{ }^{\beta}-\Sigma_{\alpha}{ }^{\beta}-\epsilon_{\alpha}{ }^{\beta}{ }_{\gamma} R^{\gamma}, \\
& 0=\boldsymbol{C}_{\alpha}{ }^{\beta} \boldsymbol{\partial}_{\beta}, \quad \boldsymbol{C}_{\alpha}{ }^{\beta}=\epsilon_{\alpha}{ }^{\gamma \beta}\left(\boldsymbol{\partial}_{\gamma}-A_{\gamma}\right)-N_{\alpha}{ }^{\beta}
\end{aligned}
$$

see [24]. In (3.4a), $\dot{U}^{\alpha}$ are the frame components of the acceleration of $\boldsymbol{\partial}_{0}$ w.r.t. the conformal metric $\mathbf{G}$, i.e., $\dot{U}=\nabla_{\partial_{0}} \partial_{0}$, where $\boldsymbol{\nabla}$ is the covariant derivative associated with $\mathbf{G}$. The variable $q$ is the negative Hubble scalar of the vector field $\partial_{0}$ in the unphysical spacetime, i.e., $q=-\frac{1}{3} \Theta$, where $\Theta=\nabla_{a} \partial_{0}^{a}$ is the expansion of $\boldsymbol{\partial}_{0}$ w.r.t. the metric $\mathbf{G}$. The object $\Sigma_{\alpha \beta}$ is the (trace-less) shear, $R_{\alpha}$ is the Fermi-rotation, which describes how the frame rotates w.r.t. a Fermi propagated frame, associated with the vector $\partial_{0}$ and the conformal metric G. In $(3.4 \mathrm{~b}), N^{\alpha \beta}$ and $A_{\alpha}$ are spatial commutator functions that describe the three-curvature of $\mathbf{G}$, see below; we also refer to $[12,24]$ where the analogous non-normalized objects are described. 
Using the analog of (3.4a) in the physical spacetime, i.e., $\left[\mathbf{e}_{0}, \mathbf{e}_{\alpha}\right]=\dot{u}_{\alpha} \mathbf{e}_{0}+$ $f_{\alpha}{ }^{\beta} \mathbf{e}_{\beta}$, where the trace of $f_{\alpha}{ }^{\beta}$ equals $-3 H$, cf. [24], it is straightforward to derive the equation

$$
\partial_{0} H=-(1+q) H .
$$

We thus see that, in terms of the physical spacetime, $q$ is interpreted as the deceleration parameter associated with $\mathbf{e}_{0}=\mathbf{u}$ and $\mathbf{g}$. As we will describe below, $q$ can be algebraically determined by the Raychaudhuri equation. Analogously, we define the spatial Hubble gradient $r_{\alpha}$ by

$$
\partial_{\alpha} H=-r_{\alpha} H
$$

where $r_{\alpha}$ is generically determined by the Codazzi constraint, see below. For dimensional reasons, the equations (3.5) for $H$ decouple from the remaining dimensionless equations (3.6) to (3.8), which are given next.

We are now in a position to describe the dynamics of Einstein's vacuum field equations in terms of the conformal Hubble-normalized variables introduced above. From the Hubble-normalized commutator equations, the Einstein field equations, and the Jacobi identities, see [24], we obtain a dimensionless system of coupled equations; we split the system into gauge equations, evolution equations, and constraint equations:

Gauge equation:

$$
\dot{U}_{\alpha}=\partial_{\alpha} \ln \mathcal{N}
$$

Evolution equations:

$$
\begin{aligned}
\partial_{0} E_{\alpha}{ }^{i} & =F_{\alpha}{ }^{\beta} E_{\beta}{ }^{i}, \\
\partial_{0} \Sigma_{\alpha \beta} & =-(2-q) \Sigma_{\alpha \beta}-2 \epsilon^{\gamma \delta}\left\langle\alpha \Sigma_{\beta\rangle \gamma} R_{\delta}-{ }^{3} \mathcal{S}_{\alpha \beta}+\left(I_{\Sigma}\right)_{\alpha \beta},\right. \\
\partial_{0} N^{\alpha \beta} & =\left(3 q \delta_{\gamma}{ }^{(\alpha}-2 F_{\gamma}{ }^{(\alpha}\right) N^{\beta) \gamma}+\left(I_{N}\right)^{\alpha \beta}, \\
\partial_{0} A_{\alpha} & =F_{\alpha}{ }^{\beta} A_{\beta}+\left(I_{A}\right)_{\alpha} .
\end{aligned}
$$

Constraint equations:

$$
\begin{aligned}
& 0=C_{\alpha}{ }^{\beta} E_{\beta}{ }^{i}, \\
& 0=1-\Sigma^{2}-\Omega_{k}-\left(I_{G}\right), \\
& 0=-3 A_{\beta} \Sigma_{\alpha}{ }^{\beta}+\epsilon_{\alpha}{ }^{\beta \gamma} \Sigma_{\beta}{ }^{\delta} N_{\delta \gamma}+\left(I_{C}\right)_{\alpha}, \\
& 0=A_{\beta} N_{\alpha}{ }^{\beta}+\left(I_{J}\right)_{\alpha}, \\
& 0=\left(I_{J \omega}\right)_{\alpha} .
\end{aligned}
$$


Here, $(\ldots)$ and $\langle\ldots\rangle$ denote symmetrization and trace free symmetrization, respectively; let us briefly comment on this system: equations (3.6), (3.7a), and (3.8a) are obtained from the commutator equations; (3.7b) is the trace-free Einstein evolution equations, while the remaining Einstein evolution equation, which can be written as the Raychaudhuri equation, yields the expression for $q$ that is given below in $(3.9 \mathrm{a})$; $(3.8 \mathrm{~b})$ is the Gauss constraint; (3.8c) are the Codazzi constraint equations; (3.7c), (3.7d), (3.8d), (3.8e) are obtained from the Jacobi identities.

In these equations we have employed the following definitions:

$$
\begin{aligned}
\Sigma^{2} & :=\frac{1}{6} \Sigma_{\alpha \beta} \Sigma^{\alpha \beta}, \quad q=2 \Sigma^{2}+\left(I_{q}\right), \\
\Omega_{k} & :=-\frac{1}{6}{ }^{3} \mathcal{R}, \quad{ }^{3} \mathcal{R}=-6 A^{2}-\frac{1}{2} B_{\alpha}{ }^{\alpha}+\left(I_{R}\right), \\
{ }^{3} \mathcal{S}_{\alpha \beta} & =2 A_{\gamma} N_{\delta\langle\alpha} \epsilon_{\beta\rangle}{ }^{\gamma \delta}+B_{\langle\alpha \beta\rangle}+\left(I_{S}\right)_{\alpha \beta}, \quad B_{\alpha \beta}=2 N_{\alpha}{ }^{\gamma} N_{\gamma \beta}-N_{\gamma}{ }^{\gamma} N_{\alpha \beta}
\end{aligned}
$$

here and in the following the norm of a spatial vector $V^{\alpha}$ is written as $V_{\alpha} V^{\alpha}=V^{2} ;{ }^{3} \mathcal{R},{ }^{3} \mathcal{S}_{\alpha \beta}$ are the conformal scalar curvature and trace-free Ricci curvature, respectively. Finally, the expressions for $\left(I_{*}\right)$.., which are zero in the symmetry-adapted SH case, are given by

$$
\begin{aligned}
\left(I_{\Sigma}\right)_{\alpha \beta}= & -\epsilon^{\gamma \delta}{ }_{\langle\alpha} N_{\beta\rangle \delta}\left(\dot{U}_{\gamma}-2 r_{\gamma}\right)+\left(\boldsymbol{\partial}_{\langle\alpha}+A_{\langle\alpha}\right)\left(\dot{U}_{\beta\rangle}+2 r_{\beta\rangle}\right) \\
& +\dot{U}_{\langle\alpha} \dot{U}_{\beta\rangle}-2 r_{\langle\alpha} r_{\beta\rangle}, \\
\left(I_{q}\right)= & -\frac{1}{3}\left(\boldsymbol{\partial}_{\alpha}+\dot{U}_{\alpha}-2 A_{\alpha}+2 r_{\alpha}\right)\left(\dot{U}^{\alpha}+r^{\alpha}\right), \\
\left(I_{N}\right)^{\alpha \beta}= & \left(\boldsymbol{\partial}_{\gamma}+\dot{U}_{\gamma}\right) \epsilon^{\gamma \delta(\alpha} F_{\delta}^{\beta)}, \quad\left(I_{A}\right)_{\alpha}=\frac{1}{2}\left(\boldsymbol{\partial}_{\beta}+\dot{U}_{\beta}\right)\left(3 q \delta_{\alpha}{ }^{\beta}-F_{\alpha}{ }^{\beta}\right) \\
\left(I_{G}\right)= & \frac{1}{3}\left(2 \boldsymbol{\partial}_{\alpha}-4 A_{\alpha}+r_{\alpha}\right) r^{\alpha}, \quad\left(I_{C}\right)_{\alpha}=\boldsymbol{\partial}_{\beta} \Sigma_{\alpha}{ }^{\beta}+\left(2 \delta_{\alpha}{ }^{\beta}+\Sigma_{\alpha}{ }^{\beta}\right) r_{\beta}, \\
\left(I_{J}\right)_{\alpha}= & -\frac{1}{2}\left(\boldsymbol{\partial}_{\beta} N_{\alpha}{ }^{\beta}+\epsilon_{\alpha}{ }^{\beta \gamma} \boldsymbol{\partial}_{\beta} A_{\gamma}\right), \quad\left(I_{J \omega}\right)_{\alpha}=\boldsymbol{C}_{\alpha}{ }^{\beta} \dot{U}_{\beta}, \\
\left(I_{S}\right)_{\alpha \beta}= & \partial_{\langle\alpha} A_{\beta\rangle}-\boldsymbol{\partial}_{\gamma} N_{\delta\langle\alpha} \epsilon_{\beta\rangle}{ }^{\gamma \delta}, \quad\left(I_{R}\right)=4 \boldsymbol{\partial}_{\alpha} A^{\alpha} .
\end{aligned}
$$

The above dimensionless coupled system of partial differential equations is associated with a state space described by the state vector

$$
\boldsymbol{X}=\left(E_{\alpha}^{i}, \Sigma_{\alpha \beta}, A_{\alpha}, N_{\alpha \beta}\right)
$$

we make the split

$$
\boldsymbol{X}=\left(E_{\alpha}^{i}\right) \oplus \boldsymbol{S}, \quad \text { where } \quad \boldsymbol{S}=\left(\Sigma_{\alpha \beta}, A_{\alpha}, N_{\alpha \beta}\right) .
$$


The Fermi rotation variables $R_{\alpha}$, which describe how the chosen frame rotates w.r.t. a Fermi-propagated frame, are gauge variables: thus $R_{\alpha}=0$ corresponds to choosing a Fermi frame while $R_{\alpha} \neq 0$ yields a rotating frame. The variable $\dot{U}_{\alpha}$ is regarded as a gauge variable determined by $\mathcal{N}$ (or, equivalently, by $N$ ); the spatial Hubble gradient $r_{\alpha}$ is generically determined by the Codazzi constraint (3.8c), however, it one is so inclined one can derive an evolution equation for $r_{\alpha}$ and include $r_{\alpha}$ into the state vector $\boldsymbol{S}$, see UEWE; sometimes it is also useful to elevate $q$ to a dependent variable, see $[21]$.

\subsection{Iwasawa frame variables}

In this paper we choose the conformal orthonormal frame $\boldsymbol{\partial}_{\alpha}=E_{\alpha}{ }^{i} \partial_{x^{i}}$ to be a conformally rescaled Iwasawa frame, i.e., $E_{\alpha}{ }^{i}=H^{-1} e_{\alpha}{ }^{i}$, where $e_{\alpha}{ }^{i}$ is a lower triangular matrix ${ }^{4}$ with the diagonal entries written in an exponential form, see Section 2.

In Appendix A, we show that choosing a conformal Iwasawa frame in the conformal Hubble-normalized dynamical systems approach corresponds to choosing the gauge

$$
\Sigma_{23}=-R_{1}, \quad \Sigma_{31}=R_{2}, \quad \Sigma_{12}=-R_{3},
$$

and setting

$$
N_{3}:=N_{33}=0
$$

This makes it natural to introduce the notation $\Sigma_{\alpha}=\Sigma_{\alpha \alpha}$, and to replace the off-diagonal components of $\Sigma_{\alpha \beta}$ by $R_{\alpha}$ according to the above equation, i.e.,

$$
\left(\begin{array}{ccc}
\Sigma_{11} & \Sigma_{12} & \Sigma_{13} \\
\Sigma_{21} & \Sigma_{22} & \Sigma_{23} \\
\Sigma_{31} & \Sigma_{32} & \Sigma_{33}
\end{array}\right)=\left(\begin{array}{ccc}
\Sigma_{1} & -R_{3} & R_{2} \\
-R_{3} & \Sigma_{2} & -R_{1} \\
R_{2} & -R_{1} & \Sigma_{3}
\end{array}\right) .
$$

By this choice of gauge we write the state vector $\boldsymbol{S}$ as

$$
\boldsymbol{S}=\left(\Sigma_{\alpha}, R_{\alpha}, A_{\alpha}, N_{\alpha \beta}\right) .
$$

For further details we refer to Appendix A, where we give the relationships between the Hamiltonian Iwasawa variables and the Hubble-

\footnotetext{
${ }^{4}$ Since $e_{\alpha}{ }^{i}=\exp \left(b^{\alpha}\right) \overline{\mathcal{N}}^{i}{ }_{\alpha}$, the components $E_{\alpha}{ }^{i}$ form a lower triangular matrix when $\overline{\mathcal{N}}^{i}{ }_{\alpha}$ is an upper triangular matrix, which is in accordance with the definitions of Section 2. For the case of the coframe, both $E_{i}^{\alpha}$ and $\mathcal{N}^{\alpha}{ }_{i}$ are upper triangular matrices.
} 
normalized variables of the conformal Hubble-normalized dynamical systems approach.

As we will see in this paper, the Iwasawa gauge has non-trivial consequences for the description of the generic asymptotic dynamics of solutions in a neighborhood of a generic spacelike singularity. However, next we describe some central concepts that are independent of the chosen frame.

\section{Asymptotic silence, locality, and the silent boundary}

\subsection{Asymptotic silence and asymptotic locality}

A generic spacelike singularity is expected to be a scalar curvature singularity $^{5}$ associated with ultra strong gravity which increasingly focuses light in all directions as the singularity is approached. The resulting asymptotic collapse of the light cones ${ }^{6}$ causes the particle horizons to shrink to zero size toward the singularity along any timeline. As a consequence, communication between different timelines is prohibited in the asymptotic limit, and we therefore refer to this causal feature of shrinking particle horizons as asymptotic silence; the associated singularity is said to be asymptotically silent.

Evidence for the conjecture that generic singularities are asymptotically silent is discussed in UEWE and [26]; we also refer to corroborative results from the context of SH cosmologies, see $[12,18,19,25]$ and references therein. In Section 4 in UEWE it is shown that, generically, asymptotic silence is connected with the property that the Hubble-normalized spatial frame variables vanish asymptotically, i.e.,

$$
E_{\alpha}^{i} \rightarrow 0
$$

toward the singularity; however, this is not sufficient for asymptotic silence, as is illustrated by special examples in [23, Section 4]. We also refer to [23] for examples of various non-generic asymptotically silent singularities and for examples of singularities for which asymptotic silence does not hold a phenomenon referred to as asymptotic silence-breaking.

\footnotetext{
${ }^{5}$ In contrast, a non-scalar curvature singularity is expected to require fine-tuning of initial data.

${ }^{6}$ We may hence refer to this as an "anti-Newtonian" limit.
} 
We define the dynamics along a timeline toward a singularity to be asymptotically local if

$$
E_{\alpha}{ }^{i} \rightarrow 0, \quad \boldsymbol{\partial}_{\alpha}\left(\boldsymbol{S}, r_{\beta}, \dot{U}_{\beta}\right) \rightarrow 0, \quad\left(r_{\alpha}, \dot{U}_{\alpha}\right) \rightarrow 0 .
$$

toward the singularity. (Note that this definition slightly differs from the one used in [22]). Asymptotic silence and asymptotic local dynamics are closely related concepts. The shrinking of particle horizons and the associated loss of communication between timelines suggests that inhomogeneities are irrelevant for the asymptotic dynamics, since they are shifted outside the shrinking horizons faster than they grow, i.e., $E_{\alpha}{ }^{i}$ goes to zero at a rate that is faster than the possible growth rate of $\partial_{i} \boldsymbol{S}, \partial_{i} \log (H), \partial_{i} \log (\mathcal{N})$. This is supported for generic timelines toward generic asymptotically silent spacelike singularities by numerical experiments $[21,22]$, but it may not be the case for all timelines. This is an issue that will be discussed in the concluding remarks in connection with "recurring spike formation" [22]; see also the discussion in [23]. However, apart from in the concluding remarks, in the remainder of this paper we will be concerned with generic timelines approaching generic asymptotically silent singularities for which the dynamics is assumed to be asymptotically local.

\subsection{The silent boundary}

Consider the Einstein field equations in the conformal Hubble-normalized approach, i.e., the system of equations (3.6) to (3.10) introduced in Section 3. It follows from (3.7a), in connection with (3.8a), that

$$
E_{\alpha}{ }^{i}=0
$$

defines an invariant subset of the state space; we refer to this invariant subset as the silent boundary, which is characterized by the state vector $\boldsymbol{S}$. Since $\boldsymbol{\partial}_{\alpha}=E_{\alpha}{ }^{i} \partial_{i}$, equation (4.2) implies $\boldsymbol{\partial}_{\alpha} \boldsymbol{S}=0$ and $\boldsymbol{\partial}_{\alpha} \dot{U}_{\beta}=0, \boldsymbol{\partial}_{\alpha} r_{\beta}=0$ in (3.6) to (3.10), so that the equations on the silent boundary reduce to a system of ordinary differential equations. Note, however, that we obtain a system of ODEs for each spatial point $x^{i}$; hence the silent boundary can be visualized as an infinite set of copies, one for each spatial point, of a finite dimensional state space. The equations on the silent boundary are identical to the equations for the state vector $\boldsymbol{S}$ of spatially self-similar and $\mathrm{SH}$ models. This is a direct consequence from the symmetry-assumptions, since spatial self-similarity implies that $\boldsymbol{\partial}_{\alpha} \boldsymbol{S}=0$ and $\boldsymbol{\partial}_{\alpha}\left(\dot{U}_{\alpha}, r_{\alpha}\right)=0$ in a symmetry adapted frame, which in turn entails that the equations for $E_{\alpha}{ }^{i}$ 
decouple, so that one obtains a reduced coupled system of ODEs for the variables $\boldsymbol{S}$.

The SH subset of the silent boundary (in brief: SH silent boundary) is defined as the invariant subset of the silent boundary given by

$$
E_{\alpha}{ }^{i}=0 \quad \text { and } \quad \dot{U}_{\alpha}=0, \quad r_{\alpha}=0 .
$$

Since this yields $\left(I_{*}\right)_{\text {.. }}=0$, the equations on the SH silent boundary reduce to a system of ordinary differential equations, which is obtained by setting the quantities $\left(I_{*}\right)$.. to zero in (3.6) to (3.10). This system is identical to the (reduced) system of equations for $\mathrm{SH}$ models, since spatial homogeneity implies $\boldsymbol{\partial}_{\alpha} \boldsymbol{S}=0$ and $\dot{U}_{\alpha}=r_{\alpha}=0$ in a symmetry-adapted frame, which subsequently leads to a decoupling of the quantities $E_{\alpha}{ }^{i}$; see also [29]. Note again that the $\mathrm{SH}$ silent boundary is an infinite set of copies, parameterized by the spatial coordinates, of a finite dimensional state space with $\boldsymbol{S}$ as state vector.

The particular importance of the SH silent boundary in the study of generic spacelike singularities stems from its connection with asymptotically local dynamics; compare (4.1) and $\left(4.2^{\prime}\right)$. The reasoning is as follows: The Hubble-normalized conformal orthonormal frame approach produces field equations that are regular in the asymptotic limit toward a generic spacelike singularity. This allows us to extend the state space to also include the silent boundary in our analysis of the dynamical system. This is highly advantageous; in particular, based on our expectation that generic spacelike singularities are asymptotically local, see (4.1), we are now able to conjecture that the asymptotic behavior of asymptotically local solutions is reflected in the dynamics on the SH silent boundary; see the discussion in Section 10. Therefore, the SH silent boundary and its neighborhood in the full state space will be the main object of investigation in this paper.

In contrast to the spatially self-similar and SH models, the variables $\boldsymbol{S}$ on the SH silent boundary depend on the spatial coordinates, and the constants of integration are therefore spatially dependent functions. The dynamical systems formalism thus explains the first step in the heuristic ad hoc procedure by BKL, which consists in replacing the constants in certain SH models with spatially dependent functions. Moreover, the ODE structure on the silent boundary in turn induces ODE structures associated with perturbations thereof - in a series expansion, spatial partial derivatives only act on spatial functions associated with previous lower order terms, thus only yielding temporal constants for the perturbative equations; this feature naturally captures the BKL statement that spatial derivatives only enters "passively" into the equations [4, pp. 656-657]. Note, however, that the dynamical 
systems approach allows one to derive the results of BKL and thus put them in the rigorous context of the regularized dimensionless state space picture of Einstein's field equations, a picture which also simplifies comparisons with analytical results for special cases as well as with numerical investigations.

The asymptotic ODE structure induced by asymptotic local dynamics can be exploited in several ways; in particular, it allows us to reparameterize the individual timelines, for which the spatial coordinates $x^{i}$ are fixed, so that $\partial_{0} f=-d f / d \tau$ along a given timeline, where $f\left(x^{0}, x^{i}\right)$ is any variable occurring in an ODE, and $\tau\left(x^{0}, x^{i}\right):=-\log (\ell / \hat{\ell})$ is a "local time function" directed toward the singularity. In this context, $\ell=g^{1 / 6}$ and $g$ is the determinant of the physical spatial metric. In our convention, hatted objects refer to objects that are functions of the spatial coordinates alone; note also that we choose a time direction toward the singularity, which is in contrast to UEWE. To obtain the solution in the chosen time coordinate $x^{0}$, one subsequently integrates the relation $d x^{0}=-\mathcal{N}^{-1} d \tau$ so that

$$
x^{0}=\hat{x}^{0}\left(x^{i}\right)-\int_{\tau_{0}}^{\tau} \mathcal{N}^{-1}\left(\tau^{\prime}, x^{i}\right) d \tau^{\prime} .
$$

The reparameterization freedom allows us to introduce a dynamical systems treatment of the equations on the silent boundary and its neighborhood, in complete analogy with the treatment of the so-called silent cosmological models from a dynamical systems perspective, see [30,31] and $\left[12\right.$, Chapter 13]. ${ }^{7}$ From now on, we will use the local reparameterization freedom induced by asymptotically local dynamics and write

$$
\partial_{0}=-\partial_{\tau},
$$

to study the asymptotic dynamics along timelines by means of finite dimensional dynamical systems techniques. ${ }^{8}$

\section{Kasner circle stability and the oscillatory subset}

It is plausible that there exists an open set of vacuum models that possess asymptotically silent singularities and obey asymptotically local dynamics for a generic set of timelines. This follows from the analysis of UEWE [20]

\footnotetext{
${ }^{7}$ In the present context it is irrelevant that the only non-trivial spatially inhomogeneous non-rotating exact silent solutions without a cosmological constant turned out to be the Szekeres dust models; see [32] and, for similar topics and additional references, [33].

${ }^{8}$ As a simple illustration, we derive the generalized Kasner line element of BKL in Appendix B.
} 
in combination with the results of [26], where a dynamical systems formulation was used that added the null geodesic equations to the Einstein field equations; further support comes from the numerical analysis of [21] and from $[22,23]$. We therefore conclude that at a generic spatial point the $\mathrm{SH}$ silent boundary - or rather a subset of the SH silent boundary - constitutes a local attractor for solutions in the full inhomogeneous state space associated with the Einstein equations; note, however, that it can only be a local attractor in the full state space since there exists, e.g., an open set of solutions without any singularity at all [34]; moreover, there may exist an open set of solutions with weak null singularities, see [35] and references therein, or an open set of solutions with a singularity that consists of parts that are asymptotically silent and spacelike and parts that are weak null singular; for special examples of singularities of this latter type, see [23].

The main aim of the present paper is to identify the subset of the SH silent boundary that acts as the attractor for generic asymptotic dynamics. As suggested by the analysis of $\mathrm{SH}$ models, the subsets that represent Bianchi type I and II models will be of crucial importance. The common denominator of these models is the most fundamental structure of the $\mathrm{SH}$ silent boundary state space: the Kasner circle.

\subsection{The Kasner circle, stable variables, and the oscillatory subset}

The SH part of the silent boundary $E_{\alpha}{ }^{i}=0$ is given by $\dot{U}_{\alpha}=r_{\alpha}=0$; it consists of an infinite number of identical copies of the finite dimensional state space spanned by the state vector $\boldsymbol{S}=\left(\Sigma_{\alpha \beta}, A_{\alpha}, N_{\alpha \beta}\right)$; hereby, the spatial variables can be regarded as acting as an index set. The associated system of evolution equations and constraints is obtained when the quantities $\left(I_{*}\right)$. are set to zero in $(3.7 \mathrm{~b})$ to $(3.7 \mathrm{~d})$ and $(3.8 \mathrm{~b})$ to $(3.8 \mathrm{~d})$, respectively. This system of equations possesses a one-parameter set of equilibrium points which is fundamental for our analysis: the Kasner circle $\mathrm{K}^{\bigcirc}$. It is determined by

$$
1-\Sigma^{2}=A_{\alpha}=N_{\alpha \beta}=R_{\alpha}=0 .
$$

Since $R_{\alpha}=0$ corresponds to $\Sigma_{\alpha \beta}=0(\alpha \neq \beta), \Sigma_{\alpha \beta}$ is diagonal for every point on $\mathrm{K}^{\bigcirc}$, i.e., $\Sigma_{\alpha \beta}=\operatorname{diag}\left[\hat{\Sigma}_{1}, \hat{\Sigma}_{2}, \hat{\Sigma}_{3}\right]$, where the numbers $\hat{\Sigma}_{\alpha}(\alpha=1, \ldots, 3)$ are constants. (All considerations apply to each of the (infinitely many) copies of the finite dimensional SH state space. Since the numbers $\hat{\Sigma}_{\alpha}$ $(\alpha=1, \ldots, 3)$ may differ between the individual copies of the SH state space, $\hat{\Sigma}_{\alpha}$ are in fact spatially dependent functions. In this context recall that we employ hatted objects to denote temporally constant spatially dependent 
functions.) It is standard to represent the Kasner circle in terms of the (generalized, when viewed as spatially dependent) Kasner exponents $p_{\alpha}$,

$$
\Sigma_{\alpha \beta}=\operatorname{diag}\left[\hat{\Sigma}_{1}, \hat{\Sigma}_{2}, \hat{\Sigma}_{3}\right]=\operatorname{diag}\left[3 p_{1}-1,3 p_{2}-1,3 p_{3}-1\right],
$$

where we have omitted the hats on top of $p_{\alpha}$ in order to agree with standard notation. Since $\operatorname{tr} \Sigma_{\alpha \beta}=0$ and $\Sigma^{2}=1$, the Kasner exponents satisfy the Kasner relations

$$
p_{1}+p_{2}+p_{3}=1, \quad p_{1}^{2}+p_{2}^{2}+p_{3}^{2}=1
$$

In Appendix B we derive the generalized Kasner line element, see [4],

$$
d s^{2}=-d t^{2}+\left(t^{2 p_{1}} l_{i} l_{j}+t^{2 p_{2}} m_{i} m_{j}+t^{2 p_{3}} n_{i} n_{j}\right) d x^{i} d x^{j}
$$

from the fixed point solution given by (5.1) and (5.2).

The Kasner circle $\mathrm{K}^{\bigcirc}$ can be divided into six sectors, where each sector is characterized by a typical ordered sequence of the Kasner exponents $\left(p_{\alpha}\right)$; hence, sector (123) is defined as the part of $\mathrm{K}^{\bigcirc}$ where $p_{1}<p_{2}<p_{3}$, whereas sector (312) is characterized by the order $p_{3}<p_{1}<p_{2}$, etc.; see figure 1(a). Evidently, the six sectors can be identified with each other through permutations of the spatial axes. Of particular interest are the six special points on the Kasner circle that are associated with solutions possessing an additional symmetry, the so-called locally rotationally symmetric (LRS) solutions. The points $Q_{\alpha}$ correspond to the three equivalent LRS solutions whose intrinsic geometry is non-flat; for $Q_{1}$ we have $\left(\hat{\Sigma}_{1}, \hat{\Sigma}_{2}, \hat{\Sigma}_{3}\right)=$ $(-2,1,1)$ and $\left(p_{1}, p_{2}, p_{3}\right)=\left(-\frac{1}{3}, \frac{2}{3}, \frac{2}{3}\right)$, cyclic permutations yield the parameters for $Q_{2}$ and $Q_{3}$. Each point $T_{\alpha}$ corresponds to a flat LRS solution: the Taub representation of Minkowski spacetime; the Taub points are given by $\left(\hat{\Sigma}_{1}, \hat{\Sigma}_{2}, \hat{\Sigma}_{3}\right)=(2,-1,-1)$ or $\left(p_{1}, p_{2}, p_{3}\right)=(1,0,0)$, and cyclic permutations; see Figure 1(a).

It is convenient to parameterize the Kasner exponents in terms of the Kasner parameter $u$, which can be defined frame invariantly through

$$
\operatorname{det}\left(\Sigma_{\alpha \beta}\right)=2-\frac{27 u^{2}(1+u)^{2}}{\left(1+u+u^{2}\right)^{3}}, \quad u \in[1, \infty]
$$

see Appendix C for details. Due to frame invariance, the Kasner parameter naturally captures the equivalence of the six sectors of $\mathrm{K}^{\bigcirc}$, see figure $1(\mathrm{~b})$. 


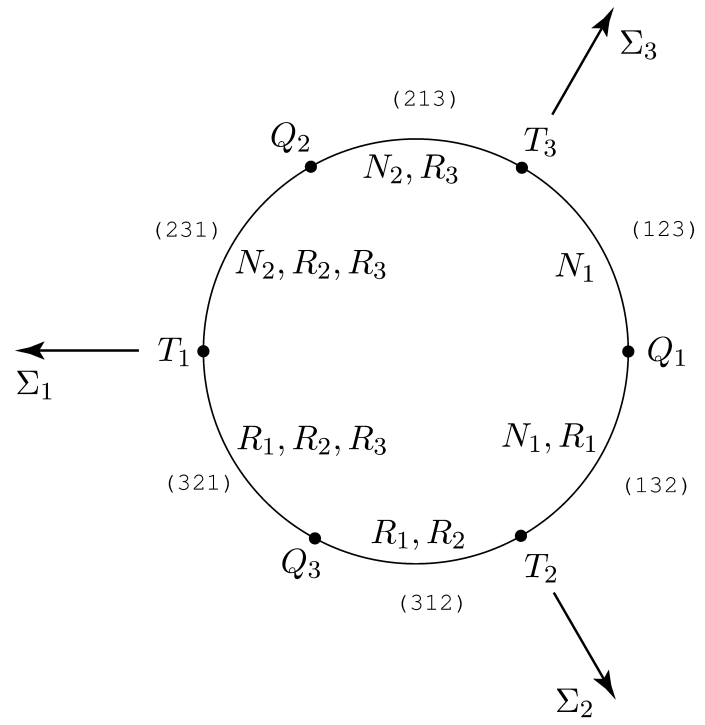

(a) Kasner sectors and unstable variables.

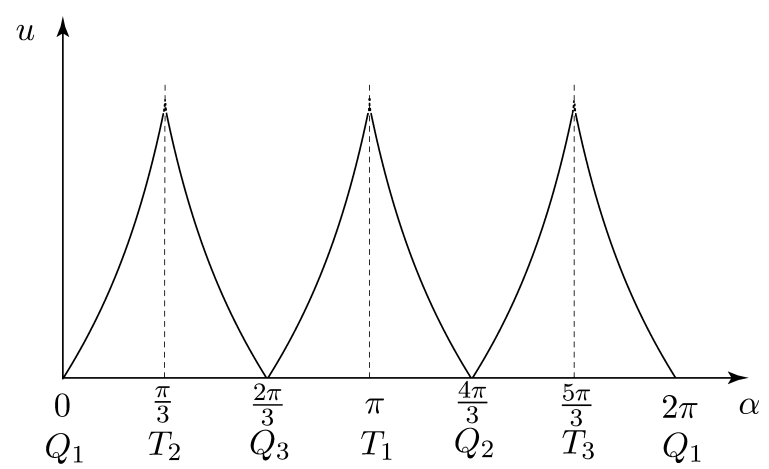

(b) Variation of the Kasner parameter $u$ along the Kasner circle.

Figure 1: The Kasner circle of fixed points; sectors and unstable variables and relation to the Kasner parameter $u$. Note that $u=1$ at the $Q_{\alpha}$ points and that $u=\infty$ at the Taub points $T_{\alpha}$.

On sector $(\alpha, \beta, \gamma)$, where $p_{\alpha}<p_{\beta}<p_{\gamma}$, we set

$$
p_{\alpha}=\frac{-u}{1+u+u^{2}}, \quad p_{\beta}=\frac{1+u}{1+u+u^{2}}, \quad p_{\gamma}=\frac{u(1+u)}{1+u+u^{2}}, \quad u \in(1, \infty) .
$$

It is easy to check that $p_{\alpha}+p_{\beta}+p_{\gamma}=1$ and $p_{\alpha}^{2}+p_{\beta}^{2}+p_{\gamma}^{2}=1$. The parameter $u=1$ describes the points $\mathrm{Q}_{1}, \mathrm{Q}_{2}, \mathrm{Q}_{3}$, i.e., the three equivalent 
representations of the non-flat LRS Kasner solution, while $u=\infty$ defines the Taub points $\mathrm{T}_{1}, \mathrm{~T}_{2}, \mathrm{~T}_{3}$ and thus the Taub solution. ${ }^{9}$

We proceed by performing a local dynamical systems analysis in a neighborhood of the Kasner circle $\mathrm{K}^{\bigcirc}$. Linearization of the dynamical system at an arbitrary point $\left(p_{1}, p_{2}, p_{3}\right)$ of $\mathrm{K}^{\bigcirc}$ yields

$$
\partial_{\tau} E_{\alpha}{ }^{i}=-3\left(1-p_{\alpha}\right) E_{\alpha}{ }^{i} \quad(\text { no sum over } \alpha)
$$

for the conformal frame variables, and

$$
\begin{aligned}
\partial_{\tau} A_{\alpha} & =-3\left(1-p_{\alpha}\right) A_{\alpha} \quad(\text { no sum over } \alpha) \\
\partial_{\tau} N_{\alpha \beta} & =-6\left(1-p_{\gamma}\right) N_{\alpha \beta} \quad(\alpha \neq \beta \neq \gamma \neq \alpha),
\end{aligned}
$$

for the variables $A_{\alpha}, N_{\alpha \beta}(\alpha \neq \beta)$ on the SH silent boundary; furthermore,

$$
\begin{aligned}
& \partial_{\tau} N_{1}=-6 p_{1} N_{1}, \quad \partial_{\tau} N_{2}=-6 p_{2} N_{2} \\
& \partial_{\tau} R_{1}=3\left(p_{2}-p_{3}\right) R_{1}, \quad \partial_{\tau} R_{2}=-3\left(p_{3}-p_{1}\right) R_{2}, \quad \partial_{\tau} R_{3}=3\left(p_{1}-p_{2}\right) R_{3}
\end{aligned}
$$

where we set $N_{1}=N_{11}$ and $N_{2}=N_{22}$ (recall that $\left.N_{3}=N_{33}=0\right)$. We see that the variables $E_{\alpha}^{i}, N_{\alpha \beta}(\alpha \neq \beta)$, and $A_{\alpha}$ belong to the stable subspace of each fixed point of $\mathrm{K}^{\bigcirc}$ (except for the Taub points). In contrast, the variables $\left(R_{1}, R_{2}, R_{3}\right)$ and $\left(N_{1}, N_{2}\right)$ are stable or unstable depending on the sector of $\mathrm{K}^{\bigcirc}$ the point $\left(p_{1}, p_{2}, p_{3}\right)$ lies in. Finally, the variables $\Sigma_{\alpha}=\Sigma_{\alpha \alpha}$ belong to the center subspace, i.e., they are constant to first order. The analysis of the stability of the Kasner circle $\mathrm{K}^{\bigcirc}$ is summarized in figure 1(a), where the unstable variables are given for each sector of $\mathrm{K}^{\bigcirc}$.

We now decompose the state vector $\boldsymbol{S}=\left(\Sigma_{\alpha \beta}, A_{\alpha}, N_{\alpha \beta}\right)$ of the SH silent boundary into a "stable" and an "oscillatory" part: $\boldsymbol{S}=\boldsymbol{S}_{\text {stable }} \oplus \boldsymbol{S}_{\mathrm{osc}}$, where $\boldsymbol{S}_{\text {stable }}=\left(N_{\alpha \beta}, A_{\alpha}\right)(\alpha \neq \beta)$ and $\boldsymbol{S}_{\mathrm{osc}}=\left(\Sigma_{\alpha}, R_{\alpha}, N_{1}, N_{2}\right)$. The subset determined by $\boldsymbol{S}_{\text {stable }}=0$ on the SH silent boundary is an invariant subset, which we call the oscillatory subset $\mathcal{O}$.

We conjecture that there exists an open set of solutions whose behavior is governed by the Kasner states as $\tau \rightarrow \infty$, i.e., we expect solutions to spend an increasing amount of time close to $\mathrm{K}^{\bigcirc}$ as $\tau \rightarrow \infty$. Equations (5.5b) and (5.5c) suggest that the variables $E_{\alpha}{ }^{i}$ and the stable variables $\boldsymbol{S}_{\text {stable }}=$

\footnotetext{
${ }^{9}$ For the BKL definition of $u$, the order of $p_{\alpha}$ is fixed according to $p_{1} \leq p_{2} \leq p_{3}$; we have found it convenient to use the above frame-independent definition instead, and to permute the ordering of $p_{\alpha}$ according to the sector one considers when dealing with framedependent matters.
} 
$\left(N_{\alpha \beta}, A_{\alpha}\right)(\alpha \neq \beta)$ decay rapidly, and that $\left(E_{\alpha}{ }^{i}, \boldsymbol{S}_{\text {stable }}\right) \rightarrow 0$ in the limit $\tau \rightarrow \infty$; this motivates the notation "stable". In anticipation of results to come, we note that the behavior of the variables $S_{\text {osc }}=\left(\Sigma_{\alpha}, R_{\alpha}, N_{1}, N_{2}\right)$ on the oscillatory subset, by contrast, can be best described as "oscillatory", whence the chosen nomenclature. Since we conjecture $\left(E_{\alpha}{ }^{i}, \boldsymbol{S}_{\text {stable }}\right) \rightarrow 0$, it is the complicated dynamics of $\boldsymbol{S}_{\text {osc }}$ on the oscillatory subset $\mathcal{O}$ that will play the decisive role in our description of the generic asymptotic dynamics - we thus begin by closely investigating the dynamical system on $\mathcal{O}$.

\subsection{The oscillatory dynamical system}

Recall from Section 4 that the silent boundary contains an infinite number of identical copies of the SH state space and thus of the oscillatory state space $\mathcal{O}$; hereby, the spatial variables act as the index set. Although it is essential for our ultimate aims to consider this infinite collection of spaces $\mathcal{O}$, this is irrelevant for our proximate purposes: in the present context we may regard the oscillatory subset $\mathcal{O}$ as one finite dimensional state space.

The evolution equations on the oscillatory subset $\mathcal{O}$ are given by setting the stable variables to zero in equations $(3.7 \mathrm{~b})$ and $(3.7 \mathrm{c})$, i.e., $\boldsymbol{S}_{\text {stable }}=$ $\left(N_{\alpha \beta}, A_{\alpha}\right)=0(\alpha \neq \beta)$. (Recall that the quantities $\left(I_{*}\right)$.. are zero because $\mathcal{O}$ is a subset of the SH silent boundary determined by $E_{\alpha}{ }^{i}=0, \dot{U}_{\alpha}=r_{\alpha}=0$.) Accordingly, the dynamical system on $\mathcal{O}$ takes the form

$$
\begin{aligned}
& \partial_{\tau} \Sigma_{1}=2\left(1-\Sigma^{2}\right) \Sigma_{1}-2\left(R_{2}^{2}+R_{3}^{2}\right)+{ }^{3} \mathcal{S}_{11}, \\
& \partial_{\tau} \Sigma_{2}=2\left(1-\Sigma^{2}\right) \Sigma_{2}-2\left(R_{1}^{2}-R_{3}^{2}\right)+{ }^{3} \mathcal{S}_{22}, \\
& \partial_{\tau} \Sigma_{3}=2\left(1-\Sigma^{2}\right) \Sigma_{3}+2\left(R_{1}^{2}+R_{2}^{2}\right)+{ }^{3} \mathcal{S}_{33}, \\
& \partial_{\tau} R_{1}=2\left(1-\Sigma^{2}\right) R_{1}+\left(\Sigma_{2}-\Sigma_{3}\right) R_{1}+2 R_{2} R_{3}, \\
& \partial_{\tau} R_{2}=2\left(1-\Sigma^{2}\right) R_{2}-\left(\Sigma_{3}-\Sigma_{1}\right) R_{2}, \\
& \partial_{\tau} R_{3}=2\left(1-\Sigma^{2}\right) R_{3}+\left(\Sigma_{1}-\Sigma_{2}\right) R_{3}-2 R_{1} R_{2}, \\
& \partial_{\tau} N_{1}=-2\left(\Sigma^{2}+\Sigma_{1}\right) N_{1}, \\
& \partial_{\tau} N_{2}=-2\left(\Sigma^{2}+\Sigma_{2}\right) N_{2},
\end{aligned}
$$

where

$$
\begin{aligned}
\Sigma^{2} & =\frac{1}{6}\left(\Sigma_{1}^{2}+\Sigma_{2}^{2}+\Sigma_{3}^{2}+2 R_{1}^{2}+2 R_{2}^{2}+2 R_{3}^{2}\right), \\
{ }^{3} \mathcal{S}_{11} & =\frac{1}{3}\left(N_{1}-N_{2}\right)\left(2 N_{1}+N_{2}\right), \\
{ }^{3} \mathcal{S}_{22} & =-\frac{1}{3}\left(N_{1}-N_{2}\right)\left(N_{1}+2 N_{2}\right), \quad{ }^{3} \mathcal{S}_{33}=-\frac{1}{3}\left(N_{1}-N_{2}\right)^{2} .
\end{aligned}
$$


The Gauss constraint (3.8b) becomes

$$
1-\Sigma^{2}-\frac{1}{12}\left(N_{1}-N_{2}\right)^{2}=0,
$$

while the Codazzi constraints (3.8c) result in

$$
N_{1} R_{2}=0, \quad N_{1} R_{3}=0, \quad N_{2} R_{1}=0, \quad N_{2} R_{3}=0 .
$$

Note that — a priori — the Codazzi constraints read

$$
N_{1} R_{2}=0, \quad N_{2} R_{1}=0, \quad\left(N_{1}-N_{2}\right) R_{3}=0 ;
$$

however, unless $N_{1} \equiv N_{2} \neq 0$ on a finite $\tau$-interval, the constraints (5.6k) ensue. Now assume that $N_{1} \equiv N_{2} \neq 0$, so that $1-\Sigma^{2}=0$; then $R_{1}=0$ and $R_{2}=0$, the equations (5.6a) to (5.6h) imply $\Sigma_{1} \equiv \Sigma_{2}$ (and $\partial_{\tau} \Sigma_{1}=\partial_{\tau} \Sigma_{2}$ ), whereby $R_{3} \equiv 0$. Therefore, $N_{1} R_{3}=0$ and $N_{2} R_{3}=0$, and (5.6k) holds in the case $N_{1} \equiv N_{2} \neq 0$ as well.

The constraints determine the structure of the oscillatory state space $\mathcal{O}$ : it consists of a number of invariant subsets ("components") that are connected with each other by parts of their boundaries only. This partitioning of $\mathcal{O}$ is to be understood in terms of the Bianchi classification: the invariant subset given by $\left(N_{1}=0\right) \wedge\left(N_{2}=0\right)$ is the Bianchi type I state space, the components $\left(N_{1}=0\right) \wedge\left(N_{2} \neq 0\right)$ and $\left(N_{1} \neq 0\right) \wedge\left(N_{2}=0\right)$ describe Bianchi type II states, and the components $N_{1} N_{2}<0$ and $N_{1} N_{2}>0$ are the Bianchi type $\mathrm{VI}_{0}$ and $\mathrm{VII}_{0}$ subsets, respectively. In other words, the equation system (5.6), when restricted to the respective component, is identical to the equations for the Bianchi type $\mathrm{I}, \mathrm{II}, \mathrm{VI}_{0}, \mathrm{VII}_{0}$ vacuum models. In the following, we more closely examine these "Bianchi components" of $\mathcal{O}$.

The Bianchi type I component is defined by $\left(N_{1}=0\right)$ and $\left(N_{2}=0\right)$, and hence $\left(R_{1}, R_{2}, R_{3}\right)$ are arbitrary, but subject to the Gauss constraint. Every orbit on the Bianchi type I component describes a Kasner solution, since

$$
\dot{U}^{2}=r^{2}=0 \quad \text { and } \quad 1-\Sigma^{2}=A^{2}=N_{\alpha \beta} N^{\alpha \beta}=0
$$

on this set; we therefore denote the Bianchi type I component alternatively as the silent Kasner subset $\mathcal{K}$. However, since $\left(R_{1}, R_{2}, R_{3}\right) \neq(0,0,0)$ (except for the fixed points on $\mathrm{K}^{\bigcirc}$ ), every orbit on $\mathcal{K}$ is a representation of a Kasner solution in a frame that rotates w.r.t. a Fermi-propagated frame; this is a consequence of the Iwasawa frame choice; see Appendix $\mathrm{C}$ for details. 
We distinguish several invariant Bianchi type I subsets which are defined by the vanishing of different combinations of the three variables $\left(R_{1}, R_{2}, R_{3}\right)$. We will use $\mathcal{B}$ as a kernel letter for an invariant subspace and a subscript that denotes the non-zero variables associated with the different subsets. Accordingly, $\mathcal{B}_{R_{\alpha}}$ is an invariant subset, where $R_{\alpha} \neq 0, R_{\beta}=0, R_{\gamma}=0(\alpha \neq$ $\beta \neq \gamma \neq \alpha$ ); for $\mathcal{B}_{R_{1} R_{3}}$ we have $R_{1} \neq 0, R_{2}=0, R_{3} \neq 0$; the set $\mathcal{B}_{R_{1} R_{2} R_{3}}$ is characterized by $R_{1} R_{2} \neq 0$ or $R_{2} R_{3} \neq 0$ and thus comprises the case of all three variables being non-zero. (It is easy to see that equations (5.6) prevent the set $\left(R_{1}=0\right)$ and the set $\left(R_{3}=0\right)$ from being invariant subsets.) The Kasner circle $\mathrm{K}^{\bigcirc}$ is a special subset of the Bianchi type I component; here, $\left(R_{1}, R_{2}, R_{3}\right)=(0,0,0)$. In the standard notation $\overline{\mathcal{B}}_{*}$ for the closure of a set, we find that, e.g., $\overline{\mathcal{B}}_{R_{1}}=\mathcal{B}_{R_{1}} \cup \mathrm{K}^{\bigcirc}$, or, $\mathcal{K}=\overline{\mathcal{B}}_{R_{1} R_{2} R_{3}}=\mathcal{B}_{R_{1} R_{2} R_{3}} \cup \mathcal{B}_{R_{1} R_{3}} \cup$ $\mathcal{B}_{R_{1}} \cup \mathcal{B}_{R_{2}} \cup \mathcal{B}_{R_{3}} \cup \mathrm{K}^{\bigcirc}$.

The Bianchi type II component is defined by either $N_{1}$ or $N_{2}$ being zero. The Codazzi constraints $(5.6 \mathrm{k})$ enforce $N_{\alpha} R_{\beta}=0(\alpha \neq \beta)$, i.e., the Bianchi type II component consists of four invariant subsets: $\mathcal{B}_{N_{1}}, \mathcal{B}_{N_{2}}$, $\mathcal{B}_{N_{1} R_{1}}$, and $\mathcal{B}_{N_{2} R_{2}}$. Solutions on the former subsets represent Bianchi type II models in a Fermi-propagated frame, since $R_{\alpha}=0$ for all $\alpha$; solutions on $\mathcal{B}_{N_{1} R_{1}}, \mathcal{B}_{N_{2} R_{2}}$ are representations in a frame that rotates w.r.t. a Fermi frame.

Finally, we denote the Bianchi type $\mathrm{VI}_{0}$ and $\mathrm{VII}_{0}$ subsets by $\mathcal{B}_{N_{1} N_{2}}$ - and $\mathcal{B}_{N_{1} N_{2}+}$, respectively, where the subscript denotes the sign of $N_{1} N_{2}$. The Codazzi constraints $(5.6 \mathrm{k})$ enforce $R_{\alpha}=0$ for all $\alpha$; hence all solutions on these components are represented in a Fermi-propagated frame.

In figure 2 we present a diagram containing the subsets $\mathcal{B}_{*}$ introduced above. We give the dimension of the different subsets and show how they are related to each other by setting variables to zero; the figure thus represents a contraction diagram.

We conclude this section by noting that models of Bianchi type VIII and IX are not described by the equations on the SH silent boundary we consider. This is due to the inherent incompatibility of the $\mathrm{SH}$ frames of the Bianchi type VIII and IX models with the Iwasawa frames we use throughout this paper; w.r.t. an Iwasawa frame Bianchi type VIII and IX solutions appear as inhomogeneous solutions and are thus associated with general solutions in the full interior state space $\boldsymbol{X}$. In contrast, the SH frames of Bianchi type $\mathrm{VI}_{-1 / 9}$ models are Iwasawa compatible as we shall see in Section 13. Since these models are expected to possess the same attractor as generic timelines of generic asymptotically silent models when expressed in an Iwasawa frame, it is rather these $\mathrm{SH}$ models that are of interest in the present context. 

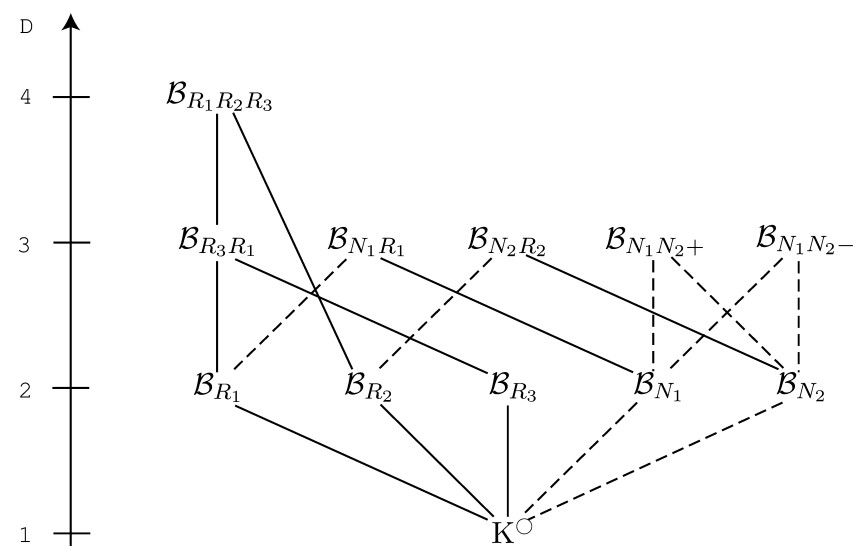

Figure 2: Subset contraction diagram of the oscillatory subset $\mathcal{O}$. In our notation, the subscripts denote the non-zero variables, and $\mathrm{D}$ hence describes the dimension of the subsets $\mathcal{B}_{*}$. Solid lines correspond to setting $R_{\alpha^{-}}$ variables to zero (frame rotation contractions); dashed lines are associated with setting $N_{1}$ or $N_{2}$ to zero (Bianchi-type contractions). Note that, e.g., $\mathcal{B}_{N_{1}}$ includes the two possible representations (one for each possible sign of $N_{1}$ ) of the Fermi-propagated subset; similar statements hold for the other subsets that involve $N_{1}$ or $N_{2}$. Analogously, e.g., $\mathcal{B}_{R_{\alpha}}$ is the union of two subsets that are characterized by the sign of $R_{\alpha}$.

\section{Dynamics on the components of the oscillatory subset}

In the following we will describe the dynamics of the system (5.6) on the oscillatory subset $\mathcal{O}$. Since $\mathcal{O}$ decomposes into independent components, we will analyze each component of $\mathcal{O}$ separately. We will see that the solutions of (5.6) are heteroclinic orbits, which, except for in the Bianchi type $\mathrm{VII}_{0}$ case, connect different fixed points on the Kasner circle and thus provide transitions between different Kasner states; accordingly we will henceforth refer to such heteroclinic orbits as transitions.

\subsection{The silent Kasner subset (Bianchi type I subset)}

The silent Kasner subset, $\mathcal{K}$, is defined as the invariant component of $\mathcal{O}$ given by $\left(N_{1}=0\right)$ and $\left(N_{2}=0\right)$ and thus by the conditions (5.7). Consequently, solutions on $\mathcal{K}$ represent Kasner states, though in general not in a Fermi frame, since $\left(R_{1}, R_{2}, R_{3}\right) \neq(0,0,0)$. In this way, a given Kasner state has several different representations on $\mathcal{K}$. 
We call the solutions of (5.6) on $\mathcal{K}$ frame transitions. Depending on how many of the variables $\left(R_{1}, R_{2}, R_{3}\right)$ are non-zero, we distinguish between single frame transitions and multiple frame transitions.

There exist three families of single frame transitions, denoted by $\mathcal{T}_{R_{1}}$, $\mathcal{T}_{R_{2}}, \mathcal{T}_{R_{3}}$, which are associated with the subsets $\mathcal{B}_{R_{1}}, \mathcal{B}_{R_{2}}, \mathcal{B}_{R_{3}}$. In the case of a $\mathcal{T}_{R_{\alpha}}$ transition, equations (5.6a) and (5.6c) yield that $\Sigma_{\alpha}=$ const and that $\Sigma_{\beta}, \Sigma_{\gamma}$ are monotonically increasing or decreasing, see figure 3 . The initial and final states of the transitions are equilibrium points on the Kasner circle $\mathrm{K}^{\bigcirc}$ that are related by $\left(\Sigma_{\alpha}\right)_{+}=\left(\Sigma_{\alpha}\right)_{-},\left(\Sigma_{\beta}\right)_{+}=\left(\Sigma_{\gamma}\right)_{-}$, and $\left(\Sigma_{\gamma}\right)_{+}=$ $\left(\Sigma_{\beta}\right)_{-}$, where the subscripts + and - denote the final and initial states of the transition, i.e., $\left(\Sigma_{\delta}\right)_{ \pm}=\lim _{\tau \rightarrow \pm \infty} \Sigma_{\delta}(\tau)$. Consequently, a $\mathcal{T}_{R_{\alpha}}$ orbit corresponds to a Kasner solution viewed in a frame that rotates around the $\alpha$-axes; in their final state, the $\beta$ - and $\gamma$-axes are rotated by $\pi / 2$ w.r.t. their initial position, which corresponds to an interchange of the $\beta$ - $/ \gamma$-directions. Since the Kasner parameter $u$ is a frame invariant, it is necessarily invariant

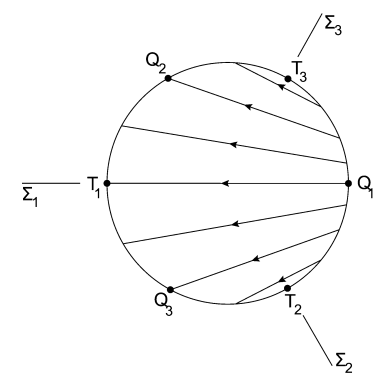

(a) $\mathcal{T}_{N_{1}}$

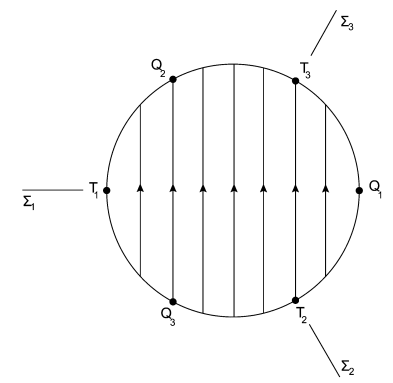

(b) $\mathcal{T}_{R_{1}}$

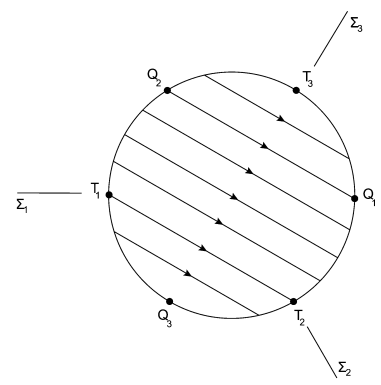

(c) $\mathcal{T}_{R_{3}}$

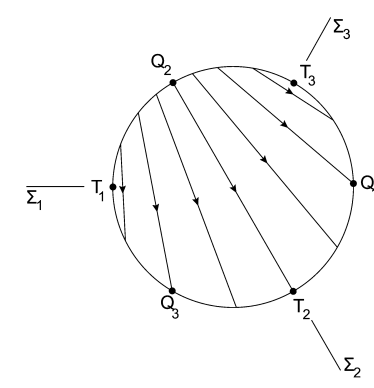

(d) $\mathcal{T}_{N_{2}}$

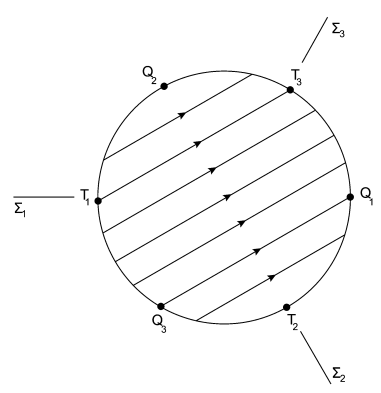

(e) $\mathcal{T}_{R_{2}}$

Figure 3: Projections of single transitions for the Iwasawa frame onto diagonal $\Sigma_{\alpha}$-space. The first three transitions, $\mathcal{T}_{N_{1}}, \mathcal{T}_{R_{1}}, \mathcal{T}_{R_{3}}$, are the transitions relevant for the billiard attractor in Section 11; the two other ones, $\mathcal{T}_{N_{2}}$, $\mathcal{T}_{R_{2}}$, are of relevance for the approach to the attractor, but asymptotically they are suppressed as shown in Section 10. 
during a frame transition; in particular,

$$
u=u_{+}=u_{-}=\text {const }
$$

see Appendix $\mathrm{C}$ for details. Expressed in $u$ we obtain for $\mathcal{T}_{R_{\alpha}}$,

$$
\left|\Delta \Sigma_{\beta}\right|=\left|\left(\Sigma_{\beta}\right)_{+}-\left(\Sigma_{\beta}\right)_{-}\right|=\left\{\begin{array}{l}
3(1+2 u)\left(1+u+u^{2}\right)^{-1} \\
3\left(2 u+u^{2}\right)\left(1+u+u^{2}\right)^{-1} \\
3\left(-1+u^{2}\right)\left(1+u+u^{2}\right)^{-1}
\end{array},\right.
$$

depending on whether the transition starts from sector $(\beta, \gamma, \alpha),(\beta, \alpha, \gamma)$, or $(\alpha, \beta, \gamma)$. A simple consequence is $\left|\Delta \Sigma_{\beta}\right| \leq 2 \sqrt{3}$.

For multiple frame transitions, more than one of the variables $\left(R_{1}, R_{2}, R_{3}\right)$ is non-zero. There exists one class of double frame transitions, $\mathcal{T}_{R_{3} R_{1}}$, which is associated with $\mathcal{B}_{R_{3} R_{1}}$, and one class of triple frame transitions, $\mathcal{T}_{R_{1} R_{2} R_{3}}$, connected with $\mathcal{B}_{R_{1} R_{2} R_{3}}$; for the latter, $R_{\alpha} \not \equiv 0 \forall \alpha$. The initial state of a multiple frame transition is an equilibrium point on sector $(321)$ of $\mathrm{K}^{\bigcirc}$, while the final state lies on (123); for details see Appendix D. The Kasner parameter $u$ is invariant under multiple frame transitions, since it is a frame invariant; furthermore, we have $\left|\Delta \Sigma_{2}\right|=0$ and $\left(\Sigma_{1}\right)_{ \pm}=\left(\Sigma_{3}\right)_{\mp}$ (as for $\mathcal{T}_{R_{2}}$ transitions). For examples of multiple frame transitions, see figure 4(a).

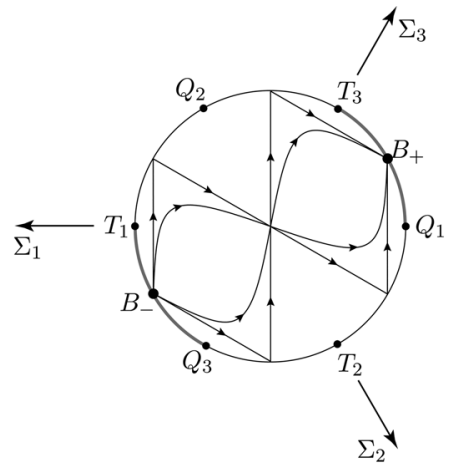

(a) Double frame transitions $\mathcal{T}_{R_{3} R_{1}}$.

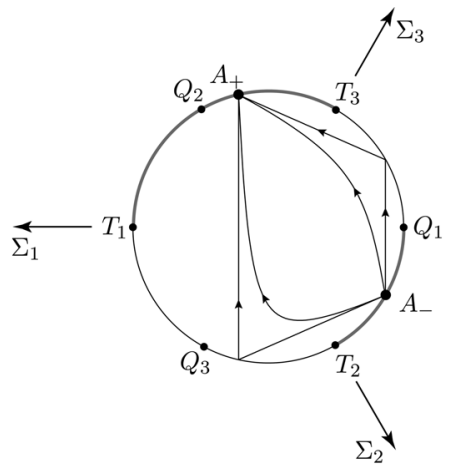

(b) Mixed frame/curvature transitions $\mathcal{T}_{N_{1} R_{1}}$.

Figure 4: (a) A projection of double frame transitions onto $\Sigma_{\alpha}$-space; note that single transitions form the boundary of this subset and that the final state of a double frame transition can be obtained in terms of a series of single transitions that are associated with the same initial Kasner state. (b) Mixed frame/curvature transitions for which analogous remarks hold. 


\subsection{The silent Bianchi type II subset}

Solutions of the oscillatory system (5.6) that satisfy $N_{\alpha} \not \equiv 0$ for some $\alpha$ are located on the Bianchi type II, $\mathrm{VI}_{0}$, or $\mathrm{VII}_{0}$ component. These solutions are characterized by non-vanishing three-curvature, i.e., ${ }^{3} \mathcal{R}=-N_{\alpha \beta} N^{\alpha \beta}+$ $\frac{1}{2}\left(N_{\alpha}^{\alpha}\right)^{2}$, see (3.9). We denote solutions of Bianchi type II and $\mathrm{VI}_{0}$ as curvature transitions; for solutions of Bianchi type $\mathrm{VII}_{0}$, we do not use this terminology for reasons that will become clear below. Curvature transitions (and Bianchi $\mathrm{VII}_{0}$ solutions) differ from frame transitions in one important respect: the Kasner parameter $u$ changes under the transition. We begin by considering those curvature transitions that are associated with the Bianchi type II subset.

The silent Bianchi type II subset is the subset of $\mathcal{O}$ determined by $\left(N_{1} \neq\right.$ $0) \wedge\left(N_{2}=0\right)$ and $\left(N_{1}=0\right) \wedge\left(N_{2} \neq 0\right)$; it is the union of the invariant subsets $\mathcal{B}_{N_{1}}, \mathcal{B}_{N_{1} R_{1}}$, and $\mathcal{B}_{N_{2}}, \mathcal{B}_{N_{2} R_{2}}$.

The simplest solutions on the silent Bianchi type II subset are the orbits on $\mathcal{B}_{N_{1}}$ or $\mathcal{B}_{N_{2}}$, denoted by $\mathcal{T}_{N_{1}}$ and $\mathcal{T}_{N_{2}}$, which we call single curvature transitions; the absence of a third family, $\mathcal{T}_{N_{3}}$ is due to the peculiarities of the Iwasawa frame. In the following we consider $\mathcal{T}_{N_{1}}$ transitions; the treatment of $\mathcal{T}_{N_{2}}$ transitions is analogous. For $\mathcal{T}_{N_{1}}$, the Gauss constraint (5.6j) yields $N_{1}^{2} / 12=1-\Sigma^{2}$. The resulting system for $\left(\Sigma_{1}, \Sigma_{2}, \Sigma_{3}\right)$, equations (5.6a) to (5.6c), possesses solutions whose initial states are equilibrium points on sectors (123) or (132) of $\mathrm{K}^{\bigcirc}$, see figure 3 . Without loss of generality we consider $\mathcal{T}_{N_{1}}$ transitions from sector (123) (as $\mathcal{T}_{N_{1}}$ orbits that originate from sector (132) are easily obtained by a permutation). The orbits can be parameterized by $u=u_{-}$, the Kasner parameter characterizing the initial state; in terms of an auxiliary function $\zeta(\tau)$, we obtain

$$
\Sigma_{1}=-4+\left(1+u^{2}\right) \zeta, \quad \Sigma_{2}=2-u^{2} \zeta, \quad \Sigma_{3}=2-\zeta,
$$

where the evolution equation for $\zeta$ is given by

$$
\partial_{\tau} \zeta=2\left(1-\Sigma^{2}\right) \zeta \quad \text { with } \quad\left(1-\Sigma^{2}\right)=\frac{3}{\zeta_{+} \zeta_{-}}\left(\zeta_{+}-\zeta\right)\left(\zeta-\zeta_{-}\right),
$$

where $\zeta_{ \pm}=3 /\left(1 \mp u+u^{2}\right)$; by definition, $0<\zeta_{-}<1$, and $0<\zeta_{+}<3$. The function $\zeta(\tau)$ interpolates monotonically between $\zeta_{-}($as $\tau \rightarrow-\infty)$ and $\zeta_{+}$ (as $\tau \rightarrow \infty$ ). Letting $u=1$ in (6.3) yields the orbit that connects $\mathrm{Q}_{1}$ with $\mathrm{T}_{1}$, see figure $3(\mathrm{a}) ; u=\infty$ corresponds to the point $\mathrm{T}_{3}$.

Evaluation of (6.3a) at $\tau=\mp \infty$ (corresponding to $\zeta_{\mp}$ ) yields the initial/ final states of the $\mathcal{T}_{N_{1}}$ transition, and thus a map that connects the initial 
and final Kasner states, which are described by $u=u_{-}$and $u_{+}$, respectively:

$$
u_{+}= \begin{cases}u_{-}-1 & \text { if } u_{-} \geq 2, \\ \left(u_{-}-1\right)^{-1} & \text { if } 1 \leq u_{-}<2 .\end{cases}
$$

This formula was first obtained by BKL via different methods; we will refer to (6.4) as the Kasner map. Note that this result holds for both $\mathcal{T}_{N_{1}}$ and $\mathcal{T}_{\mathrm{N}_{2}}$ curvature transitions.

For later purposes, we define the growth factor $g$ according to ${ }^{10}$

$$
g:=\frac{\zeta_{+}}{\zeta_{-}}=\frac{1+u+u^{2}}{1-u+u^{2}}=\frac{1+u_{-}+u_{-}^{2}}{1-u_{-}+u_{-}^{2}} .
$$

Since $u=u_{-} \in[1, \infty)$ for $\mathcal{T}_{N_{1}}$ orbits, we have $1<g<3$.

There exist two classes of mixed frame/curvature transitions: orbits on $\mathcal{B}_{N_{1} R_{1}}$ and orbits on $\mathcal{B}_{N_{2} R_{2}}$, which we denote by $\mathcal{T}_{N_{1} R_{1}}$ and $\mathcal{T}_{N_{2} R_{2}}$, respectively. The initial state of a $\mathcal{T}_{N_{1} R_{1}}$ orbit is an equilibrium point on sector (132), and the final state lies on (213) or (231); for $\mathcal{T}_{N_{2} R_{2}}$ transitions, the numbers 1 and 2 are interchanged; see Appendix D. In analogy to single curvature transitions, the initial and the final Kasner state are related by the Kasner map (6.4). This fact relies on the frame invariance of the parameter $u$ : $\mathcal{T}_{N_{1}}$ and $\mathcal{T}_{N_{1} R_{1}}$ transitions are representations of the same Bianchi type II solutions - they are merely expressed in different frames (Fermi frame versus rotating frame); the transformation law (6.4) of the frame invariant- $u$ is thus unaffected. For more details on mixed frame/curvature transitions, see Appendix D; for examples, see figure 4(b). Note that henceforth, for brevity, we will refer to mixed frame/curvature transitions as mixed curvature transitions.

\subsection{The silent Bianchi type $\mathrm{VI}_{0}$ and $\mathrm{VII}_{0}$ subsets}

The silent Bianchi type $\mathrm{VI}_{0}$ subset is given by $N_{1} N_{2}<0$ and $R_{\alpha}=0$ for all $\alpha$; it is denoted by $\mathcal{B}_{N_{1} N_{2}-}$. We call the orbits on $\mathcal{B}_{N_{1} N_{2}-}$ double curvature transitions: $\mathcal{T}_{N_{1} N_{2}-}$, or simply $\mathcal{T}_{N_{1} N_{2}}$. In Appendix D we show that the $\alpha$-limit of each $\mathcal{T}_{N_{1} N_{2}}$ transition is the Taub point $\mathrm{T}_{3}$; the $\omega$-limit is a fixed point on sector (312) or (321) of $\mathrm{K}^{\bigcirc}$. Accordingly, in terms of the Kasner

\footnotetext{
${ }^{10}$ The quantity $g$ should not be confused with the determinant of the spatial metric it should be clear from the context which one is meant.
} 
parameter $u$, we find

$$
u_{-}=\infty \stackrel{\mathcal{T}_{N_{1} N_{2}}\left(\text { Bianchi } \mathrm{VI}_{0}\right)}{\longrightarrow} u_{+} \in[1, \infty)
$$

note that for each $u \in[1, \infty)$ there exist double curvature transitions that map the Taub state $u_{-}=\infty$ to exactly that final Kasner state, i.e., $u=u_{+}$. It is evident that the behavior of double curvature transitions is fundamentally different from the behavior of Bianchi type II transitions (single and mixed curvature transitions); compare (6.6) with the Kasner map (6.4). We observe that there do not exist double curvature transitions with arbitrarily small amplitudes: there exists $\epsilon>0$ such that $\max _{\tau}\left|N_{1}(\tau)\right| \geq \epsilon$ (and $\max _{\tau}\left|N_{2}(\tau)\right| \geq \epsilon$ ) uniformly for all double curvature transitions; for a proof see Appendix D.

Finally, let us now consider the silent Bianchi type $\mathrm{VII}_{0}$ subset $\mathcal{B}_{N_{1} N_{2}+}$, which is defined by $N_{1} N_{2}>0$ and $R_{\alpha}=0$ for all $\alpha$. The state space contains a line of fixed points, $\mathcal{L}_{3}^{ \pm}$, given by the conditions $\left(\Sigma_{1}, \Sigma_{2}, \Sigma_{3}\right)=(1,1,-2)$ and $N_{1}=N_{2} \gtrless 0$; see Appendix D for details. The $\alpha$-limit of each Bianchi $\mathrm{VII}_{0}$ orbit is a point on $\mathcal{L}_{3}^{ \pm}$, and the $\omega$-limit is a fixed point on sector (312) or $(321)$ of $\mathrm{K}^{\bigcirc}$. Hence, since Bianchi type $\mathrm{VII}_{0}$ orbits do not connect two fixed points on $\mathrm{K}^{\bigcirc}$, we refrain from calling them transitions. For this reason, we will not introduce the nomenclature $\mathcal{T}_{N_{1} N_{2}+}$.

\section{Sequences, eras, and phases}

In the previous section, we have described the dynamics on the oscillatory subset by analyzing the solutions (transitions) on the different components of $\mathcal{O}$. These transitions form pieces of a "heteroclinic orbit puzzle" that governs asymptotically local dynamics; in the following we describe and characterize its key features.

\subsection{Sequences of transitions and Kasner sequences}

We define a sequence of transitions as a heteroclinic sequence on the oscillatory subset $\mathcal{O}$, i.e., as an infinite concatenation of transition orbits (heteroclinic orbits) on $\mathcal{O}$. (Note that, by definition, Bianchi type $\mathrm{VII}_{0}$ orbits are excluded from these sequences; since the initial states of type $\mathrm{VII}_{0}$ orbits do not lie on $\mathrm{K}^{\bigcirc}$, but on $\mathcal{L}_{3}^{ \pm}$, they cannot be concatenated with transition orbits.) Accordingly, a sequence of transitions, which we denote by $\mathcal{S}_{\mathcal{T}}$, is 
described by $\mathcal{S}_{\mathcal{T}}=\left(\mathcal{T}_{i}\right)_{i \in \mathbb{N}}=\left(\mathcal{T}_{0}, \mathcal{T}_{1}, \mathcal{T}_{2}, \ldots\right),{ }^{11}$ where each transition $\mathcal{T}_{i}$ is either a frame transition $\mathcal{T}_{R_{1}}, \mathcal{T}_{R_{2}}, \mathcal{T}_{R_{3}}, \mathcal{T}_{R_{3} R_{1}}, \mathcal{T}_{R_{1} R_{2} R_{3}}$, or a single or mixed curvature transition $\mathcal{T}_{N_{1}}, \mathcal{T}_{N_{2}}, \mathcal{T}_{N_{1} R_{1}}, \mathcal{T}_{N_{2} R_{2}}$, or a double curvature transition $\mathcal{T}_{N_{1} N_{2}}$. It is understood that the $\omega$-limit of each transition $\mathcal{T}_{i}$ (which is a fixed point on $\mathrm{K}^{\bigcirc}$ ) coincides with the $\alpha$-limit of the transition $\mathcal{T}_{i+1}$.

The initial state of each individual transition $\mathcal{T}_{i}$ is a fixed point on $\mathrm{K}^{\bigcirc}$ (which coincides with the final state of $\mathcal{T}_{i-1}$ ), and is thus associated with a particular value of the Kasner parameter $u$. Therefore, every sequence of transitions $\mathcal{S}_{\mathcal{T}}$ generates a series of Kasner parameters in a natural way. Since frame transitions do not induce any change in $u$, see (6.1), the series of Kasner states is not associated with the sequence $\mathcal{S}_{\mathcal{T}}$ directly, but rather with the sequence of curvature transitions of $\mathcal{S}_{\mathcal{T}}$, which may be regarded as its supporting "skeleton". This makes it convenient to introduce a second running index that consecutively numbers curvature transitions: $l=0,1,2, \ldots$ The index $l$ can be viewed as a function $l: \mathbb{N} \rightarrow \mathbb{N}$ such that $l(i)=l_{i}$ denotes the number of curvature transitions among the transitions $\left\{\mathcal{T}_{0}, \mathcal{T}_{1}, \ldots, \mathcal{T}_{i-1}\right\}$.

Let $u_{l}$ denote the initial Kasner state of the $l$ th curvature transition; then the transition maps $u_{l}$ to $u_{l+1}$,

$$
u_{l} \stackrel{\text { lth curvature transition }}{\longrightarrow} u_{l+1} \text {. }
$$

For single and mixed curvature transitions, the Kasner map (6.4) applies:

$$
u_{l+1}= \begin{cases}u_{l}-1 & \text { if } u_{l} \geq 2, \\ \left(u_{l}-1\right)^{-1} & \text { if } 1 \leq u_{l}<2 .\end{cases}
$$

A double curvature transition, on the other hand, maps a Kasner state characterized by $u=\infty$ to a Kasner state with $u \in(1, \infty)$.

Two cases occur: (i) The initial state $u_{0}$ is an irrational number, which is the generic case since $\mathbb{Q}$ is a set of measure zero in $\mathbb{R}$. Then the sequence $\left(u_{l}\right)_{l \in \mathbb{N}}$ is given through the recursion (7.1); in particular, for all $l, u_{l} \in$ $(1, \infty)$. A sequence $\left(u_{l}\right)_{l \in \mathbb{N}}$ of this type we denote as a Kasner sequence. Note that a Kasner sequence is associated with a sequence of transitions $\mathcal{S}_{\mathcal{T}}$ that does not contain any double curvature transitions $\mathcal{T}_{N_{1} N_{2}}$. (ii) The initial state $u_{0}$ is a rational number. Then there exists $k<\infty$ such that $u_{k-1}=1$, whereby $u_{k}=\infty$. At this point, the recursion defined by the Kasner map (7.1) is interrupted. A double curvature transition $\mathcal{T}_{N_{1} N_{2}}$ must

\footnotetext{
${ }^{11}$ In order to agree with the conventions of [3] we define the first transition of $\mathcal{S}_{\mathcal{T}}$ to carry the index number 0 .
} 
follow, which yields $u_{k+1} \in[1, \infty)$. Generically, the Kasner parameter $u_{k+1}$ is an irrational number, hence the series $\left(u_{l}\right)_{l>k}$ is a Kasner sequence given by the recursion (7.1); by a shift of origin (i.e., by redefining $u_{0}:=u_{k+1}$ ), we obtain again a standard Kasner sequence. If the Kasner parameter $u_{k+1}$ is a rational number, which is the non-generic case, we are back to square one, at the beginning of the loop.

From this discussion, we are led to classify possible sequences of transitions. Sequences with a finite number of double curvature transitions are innocuous: they are standard Kasner sequences by a simple shift of origin and thus do not require special treatment. Sequences that contain infinitely many double curvature transitions are qualitatively different, but they are highly non-generic since they emerge from repeated fine-tuning: for such sequences, the final state of each individual $\mathcal{T}_{N_{1} N_{2}}$ transition must be represented by a rational value of the Kasner parameter. This indicates that these sequences are not to be treated on an equal footing with generic sequences, i.e., sequences that are free from double curvature transitions. Henceforth, unless otherwise stated, a sequence of transitions $\mathcal{S}_{\mathcal{T}}$ always denotes a sequence where double curvature transitions are excluded, i.e., it is an infinite concatenation of frame transitions and single and mixed curvature transitions. It is sequences of this type that are intrinsically tied to Kasner sequences $\left(u_{l}\right)_{l \in \mathbb{N}}$ which are defined through the recursion (7.1).

\subsection{Eras, large curvature phases, and small curvature phases}

Here we investigate qualitative properties of Kasner sequences $\left(u_{l}\right)_{l \in \mathbb{N}}$; in particular, we introduce a partition of the sequence into phases of small curvature and phases of large curvature; however, we begin by defining the concepts of an epoch and an era.

Consider a Kasner sequence $\left(u_{l}\right)_{l \in \mathbb{N}}$ as given by the recursion (7.1). A Kasner epoch is simply defined as an individual Kasner state $u_{l}$ of the sequence. The sequence $\left(u_{l}\right)_{l \in \mathbb{N}}$ possesses a natural partition into eras: an era is a set $\left[l_{\text {in }}, l_{\text {out }}\right] \ni l$ such that $u_{l}$ is monotonically decreasing from a maximal value $u^{\text {in }}=u_{l_{\text {in }}}=\left(u_{l_{\text {in }}-1}-1\right)^{-1}$ to a minimal value $1<u^{\text {out }}=u_{l_{\text {out }}}<2$. The length of an era is given by the number of Kasner epochs it contains: $L=l_{\text {out }}-l_{\text {in }}+1$. Since $u_{l+1}=u_{l}-1$ for all $l$ of an era $\left[l_{\text {in }}, l_{\text {out }}\right]$, we have $L=\operatorname{int}\left(u^{\mathrm{in}}\right)$, where $\operatorname{int}(x)$ is a function that gives the integer part of $x$, see [5]. Note that an era can be, and many will be, of length one.

We now introduce a somewhat more flexible concept that comprises the concept of an era as a special case. Let $\mathbb{R} \ni \eta_{u} \geq 1$; we define a phase of small curvature - a small curvature phase - as a set $\left[l_{\text {in }}, l_{\text {out }}\right] \ni l$ such that $u_{l}$ monotonically decreases from a maximal value $u^{\text {in }}=u_{l_{\text {in }}}$ to a minimal 
value $u^{\text {out }}=u_{l_{\text {out }}}$ according to

$$
u^{\text {in }}=u_{l_{\text {in }}}=\left(u_{l_{\text {in }}-1}-1\right)^{-1}>\eta_{u} \geq 1 \stackrel{l \in\left[l_{\text {in }}, l_{\mathrm{out}}\right]}{\longrightarrow} \eta_{u}+1>u^{\text {out }}=u_{l_{\text {out }}}>\eta_{u} .
$$

When $\eta_{u}$ is chosen to be equal to 1 , the definition of a small curvature phase reduces to the definition of an era; however, the usefulness of the concept of a small curvature phase stems from the possibility of choosing $\eta_{u} \gg 1$, and this is the choice we will typically make. In this case, a small curvature phase contains only large values of $u$. Accordingly, the Kretschmann scalar $\mathcal{W}_{1}$ associated with the metric $\mathbf{G}$ is small, since $\mathcal{W}_{1}=27 u^{2}(1+u)^{2} /(1+u+$ $\left.u^{2}\right)^{3}=27 u^{-1}\left(1+u^{-1}+O\left(u^{-2}\right)\right)$, cf. Appendix C. Since this quantity determines the magnitude of the curvature during a Bianchi type II transition, see Section 14 and figure 9(c), the terminology "phase of small curvature" suggests itself. Finally, note that the length of a small curvature phase is defined in analogy to the length of an era as the number of Kasner epochs (i.e., Kasner parameters) it comprises:

$$
L=l_{\text {out }}-l_{\text {in }}+1=\operatorname{int}\left(u^{\text {in }}-\eta_{u}+1\right) \approx u^{\text {in }}-\eta_{u} \approx u^{\text {in }},
$$

where the last approximation holds only in the special case $u^{\text {in }} \gg \eta_{u}$.

The complement of a phase of small curvature is a phase of large curvature. A set $\left[l_{\mathrm{i}}, l_{\mathrm{f}}\right] \ni l$ is called a large curvature phase if

$$
u_{l_{\mathrm{i}}-1}>\eta_{u}, \quad u_{l} \leq \eta_{u} \text { for all } l \in\left[l_{\mathrm{i}}, l_{\mathrm{f}}\right], \quad u_{l_{\mathrm{f}}+1}>\eta_{u} .
$$

Accordingly, the length $L$ of a large curvature phase is at least $\operatorname{int}\left(u_{l_{\mathrm{i}}}\right) \geq$ $\operatorname{int}\left(\eta_{u}-1\right)$, since $u_{l_{\mathrm{i}}} \in\left(\eta_{u}-1, \eta_{u}\right)$. In a large curvature phase, the values of the Kasner parameter are comparatively small, i.e., small in comparison with the $u$-values of a small curvature phase (7.2); it follows that curvature is comparatively large in the case of a large curvature phase, see Section 14, which motivates the nomenclature "large curvature phase". Note that the concept of a large curvature phase does not exist when $\eta_{u}$ is chosen to be equal to 1 ; recall that in this case the concepts era/small curvature phase are identical.

Small curvature phases and large curvature phases occur alternately. A large curvature phase continues and ends the era that began simultaneously with the small curvature phase preceding the large curvature phase. When $1<u_{l}<2$, the Kasner parameter number $(l+1)$ begins a new era: if $u_{l+1} \leq \eta_{u}$, then that entire era belongs to the continuing large curvature phase; if, and only if, $u_{l+1}>\eta_{u}$, then a large curvature phase has ended and a new small curvature phase begins. Consequently, a large curvature phase can contain an arbitrary number of eras. In the following example, where the choice $\eta_{u}=3.5$ has been made, the large curvature phase contains two 
and a half eras.

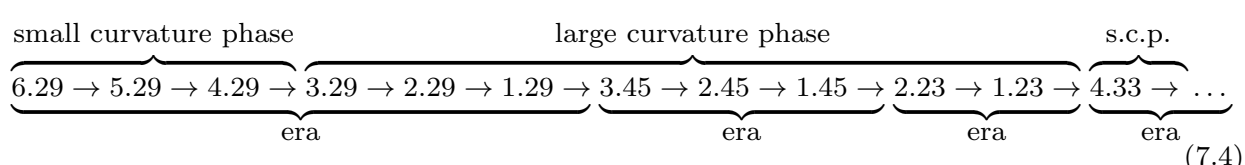

Let us now briefly discuss how curvature phases are represented in the state space $\mathcal{O}$. Consider a sequence of transitions $\mathcal{S}_{\mathcal{T}}=\left(\mathcal{T}_{i}\right)_{i \in \mathbb{N}}$ on $\mathcal{O}$ and the associated Kasner sequence $\left(u_{l}\right)_{l \in \mathbb{N}}$. Suppose that $l \in\left[l_{\text {in }}, l_{\text {out }}\right]$ is a small curvature phase (associated with a value $\eta_{u} \gg 1$ ) of the Kasner sequence $\left(u_{l}\right)_{l \in \mathbb{N}}$. Since the inverse of the Kasner parameter, i.e., $u^{-1}$, is a measure for the (angular) distance (on $\mathrm{K}^{\bigcirc}$ ) from $\mathrm{T}_{3}$ (and equivalently from $\mathrm{T}_{1}, \mathrm{~T}_{2}$ ), the condition $u^{\text {in }}>\eta_{u}$ characterizing the small curvature phase is equivalent to $\left(u^{\text {in }}\right)^{-1}<\eta_{u}^{-1}$, i.e., to the statement that the (angular) distance of the transitions from $\mathrm{T}_{3}\left(\mathrm{~T}_{1}, \mathrm{~T}_{2}\right)$ be less than a given distance $\eta_{u}^{-1}$. Suppose that the curvature transition number $l_{\text {in }}$ (which is the first curvature transition of the phase) is a $\mathcal{T}_{N_{1}}$ transition that takes place in the $\eta_{u}^{-1}$-neighborhood of the Taub point $\mathrm{T}_{3}$. In this neighborhood of $\mathrm{T}_{3}$ only three types of transitions are possible: $\mathcal{T}_{N_{1}}, \mathcal{T}_{N_{2}}$, and $\mathcal{T}_{R_{3}}$, see figure 3 ; it is thus immediate from the figures that the initial $\mathcal{T}_{N_{1}}$ transition generates a sequence where $\mathcal{T}_{N_{1}}$ transitions and $\mathcal{T}_{N_{2}}$ or $\mathcal{T}_{R_{3}}$ transitions occur alternately while the distance $u^{-1}$ from $\mathrm{T}_{3}$ slowly increases (as $u$ decreases by $u \mapsto u-1$ ). For some time, therefore, the sequence is "captured" in the $\eta_{u}^{-1}$-neighborhood of the point $\mathrm{T}_{3}$; since the curvature is small in this neighborhood, the phase is a "phase of small curvature". From (7.3) we have that the length of a captured phase is given by $L \approx u^{\text {in }}$ when $u^{\text {in }} \gg \eta_{u}$; in other words, the number of curvature transitions needed to leave the given $\eta_{u}^{-1}$-neighborhood of $\mathrm{T}_{3}$ is inversely proportional to the initial distance $\left(u^{\mathrm{in}}\right)^{-1}$ from $\mathrm{T}_{3}$. For an example of a small curvature phase in the state space picture, see figure 5.

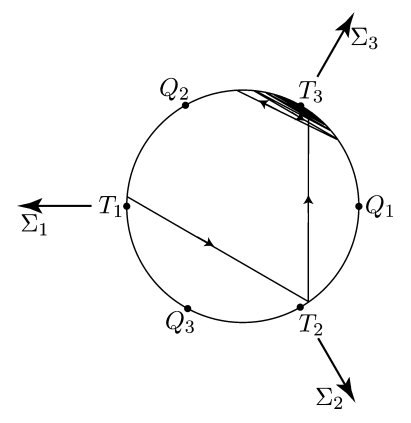

Figure 5: A small curvature phase. Note that this particular small curvature phase starts with a $\mathcal{T}_{R_{3}}$ transition followed by a $\mathcal{T}_{R_{1}}$ transition and a series of alternating $\mathcal{T}_{N_{1}}$ and $\mathcal{T}_{R_{3}}$ transitions; the latter oscillatory behavior is of particular relevance for the billiard attractor discussed in Section 11. 
In our description of generic asymptotic dynamics, it will turn out to be important to characterize Kasner sequences from a stochastic point of view. In order to do so, in the subsequent section, we will consider the space of all possible Kasner sequences.

\section{Stochastic analysis of Kasner sequences}

The recursion formula (7.1) that defines Kasner sequences typically generates sequences of pairwise different values $u_{l}$. However, there also exist Kasner sequences $\left(u_{l}\right)_{l \in \mathbb{N}}$ that are periodic; for instance, the sequence

$$
\begin{aligned}
u_{0} & =\frac{5+\sqrt{13}}{\sqrt{13}-1} \longrightarrow u_{1}=\frac{6}{\sqrt{13}-1} \longrightarrow u_{2}=\frac{7-\sqrt{13}}{\sqrt{13}-1} \longrightarrow u_{3} \\
& =u_{0}=\frac{5+\sqrt{13}}{\sqrt{13}-1} \longrightarrow \cdots
\end{aligned}
$$

is a Kasner sequence with period 3, see [37] or [12, p. 236]. Note that this Kasner sequence is associated with a heteroclinic cycle (or rather, a set of entangled heteroclinic cycles) in the state space description of sequences.

Consider an arbitrary Kasner sequence $\left(u_{l}\right)_{l \in \mathbb{N}}$. As discussed in Section 7 , this sequence possesses a natural partition into eras $j=1,2, \ldots$, where each era $j$ is associated with an initial value $u=u^{\text {in }}>1$ of the Kasner parameter, which we denote by $u_{j}^{\text {in }}$. The Kasner map (7.1) thus generates a sequence $\left(u_{j}^{\mathrm{in}}\right)_{j \in \mathbb{N}}$ obeying the recursion formula

$$
u_{j+1}^{\text {in }}=\frac{1}{\operatorname{frac}\left(u_{j}^{\text {in }}\right)},
$$

where $\operatorname{frac}(\cdot)$ is a function that gives the fractional part of its argument. It is known that the transformation (8.2) is associated with exponential instability: the distance between two points (whose initial distance is small) grows exponentially with the number of iterations $j$, see [5].

For the following, we regard the sequence $\left(u_{j}^{\text {in }}\right)_{j \in \mathbb{N}}$ as a distribution of points (given by the values of $u_{j}^{\text {in }}$ ) in the interval $(1, \infty) \subseteq \mathbb{R}$. By adopting this viewpoint, it becomes natural to ask whether this distribution possesses a probabilistic description, i.e., whether it can be modeled by a continuous probability function. In general, this will not be the case, since there exist Kasner sequences that generate finite distributions $\left\{v_{1}, \ldots, v_{n}\right\}$, i.e., $u_{j}^{\text {in }} \in$ $\left\{v_{1}, \ldots, v_{n}\right\}$ for all $j \in \mathbb{N}$. In the example (8.1) we even obtain $u_{j}^{\text {in }}=v=$ $(5+\sqrt{13}) /(\sqrt{13}-1)$ for all $j \in \mathbb{N}$. 
However, the picture changes when we consider not the distribution of one single sequence $\left(u_{j}^{\mathrm{in}}\right)_{j \in \mathbb{N}}$, but the entirety of all distributions generated by the collection of all possible Kasner sequences. This collective distribution is indeed modeled by a continuous probability function, which arises as the stationary limit from an arbitrary probability density via the map $u^{\text {in }} \mapsto$ $\left[\operatorname{frac}\left(u^{\text {in }}\right)\right]^{-1}$, cf. (8.2); see [5]. In other words, $u^{\text {in }}$ can be regarded as a random variable with a specific probability density given by

$$
w\left(u^{\text {in }}\right)=\frac{1}{\log 2} \frac{1}{u^{\text {in }}\left(1+u^{\text {in }}\right)} .
$$

According to the probabilistic description with probability density (8.3), when we choose an arbitrary $u^{\text {in }}$ from an arbitrary era of an arbitrary Kasner sequence, the probability of $u^{\text {in }}$ taking a value in $\left(v_{1}, v_{2}\right)$ is given by

$$
P\left(v_{1}<u^{\text {in }}<v_{2}\right)=\frac{1}{\log 2} \int_{v_{1}}^{v_{2}} \frac{1}{u(1+u)} d u .
$$

In order to obtain a probability distribution on a bounded interval, the inverse of the Kasner parameter $u^{\text {in }}$ is used: we denote the inverse of $u^{\text {in }}$ by $\varkappa$, i.e., $\varkappa=\left(u^{\text {in }}\right)^{-1}$; by definition, $\varkappa \in(0,1)$. The probability density of the random variable $\varkappa$ reads

$$
w(\varkappa)=\frac{1}{\log 2} \frac{1}{1+\varkappa}
$$

see [5].

It is straightforward to adapt the probabilistic description to the concept of small/large curvature phases. The Kasner sequence $\left(u_{l}\right)_{l \in \mathbb{N}}$ consists of infinitely many small curvature phases $j=1,2, \ldots$, where each small curvature phase is associated with a value $u_{j}^{\text {in }}>\eta_{u}$ of the initial Kasner parameter (which naturally coincides with the initial parameter of the era that begins simultaneously with the small curvature phase $j$, see (7.4)). Let $\varkappa_{j}$ be the inverse of $u_{j}^{\text {in }}$, i.e., $\varkappa_{j}=\left(u_{j}^{\text {in }}\right)^{-1}<\eta_{u}^{-1}$; the distribution of the sequence $\left(\varkappa_{j}\right)_{j \in \mathbb{N}}$ in the interval $\left(0, \eta_{u}^{-1}\right)$ is described by a random variable $\varkappa$ with probability density

$$
\hat{w}(\varkappa)=\frac{1}{\log \left(1+\eta_{u}^{-1}\right)} \frac{1}{1+\varkappa} ; \quad \int_{0}^{\eta_{u}^{-1}} \hat{w}(\varkappa) d \varkappa=1 ;
$$

evidently, for $\eta_{u}=1$ the distribution $\hat{w}(\varkappa)$ reduces to (8.4). Note in particular that $\hat{w}(0) \neq 0$. 
Consider a Kasner sequence $\left(u_{l}\right)_{l \in \mathbb{N}}$ whose distribution of the parameters $\left(u_{j}^{\mathrm{in}}\right)_{j \in \mathbb{N}}$ has a probabilistic description (in terms of the probability density (8.5)). For such a Kasner sequence, small curvature phases are expected to dominate over large curvature phases in the following sense: the probability that an epoch of the sequence chosen randomly among $l$ epochs belongs to a small curvature phase tends to one as $l \rightarrow \infty$. The proof is based on stochastic arguments:

We first compute the expectation value $\langle L\rangle$ of the length of a small curvature phase. Since $L \approx u^{\text {in }}-\eta_{u}=\varkappa^{-1}-\eta_{u}$, see (7.3), we obtain

$$
\langle L\rangle=\int_{0}^{\eta_{u}^{-1}} \hat{w}(\varkappa) L(\varkappa) d \varkappa \approx \hat{w}(0) \int_{0}^{\eta_{u}^{-1}}\left(\varkappa^{-1}-\eta_{u}\right) d \varkappa=\infty
$$

i.e., $\langle L\rangle$ is infinite. Note that this feature is independent of the probability density $\hat{w}(\varkappa)$ as long as $\hat{w}(0)>0$.

Second, we compute the expectation value for the length of a large curvature phase. A large curvature phase continues and ends the era that started simultaneously with the preceding small curvature phase, see (7.4). The probability for the large curvature phase to contain another (complete) era is given by

$$
p=1-\int_{0}^{\eta_{u}^{-1}} w(\varkappa) d \varkappa=\int_{\eta_{u}^{-1}}^{1} w(\varkappa) d \varkappa=1-\frac{\log \left(1+\eta_{u}^{-1}\right)}{\log 2} \approx 1-\frac{\eta_{u}^{-1}}{\log 2}<1
$$

the approximation assumes $\eta_{u} \gg 1$. Accordingly, the probability that a large curvature phase contains at least another $n$ (complete) eras is $p^{n}$; the probability that a large curvature phase contains exactly $n$ (complete) eras is $(1-p) p^{n}$. (Note here that the assumption $\eta_{u} \gg 1$ ensures that there will be sufficiently many eras in one large curvature phase to permit a probabilistic description.) The expectation value for the length of an era that is contained in a large curvature phase is

$$
\begin{aligned}
& \left(\int_{\eta_{u}^{-1}}^{1} w(\varkappa) d \varkappa\right)^{-1} \int_{\eta_{u}^{-1}}^{1} \operatorname{int}\left(\varkappa^{-1}\right) w(\varkappa) d \varkappa \approx \int_{\eta_{u}^{-1}}^{1}\left(\varkappa^{-1}-1\right) w(\varkappa) d \varkappa \\
& \quad \approx(\log 2)^{-1} \log \eta_{u},
\end{aligned}
$$

where again $\eta_{u} \gg 1$ is assumed. Accordingly, a sequence of $n$ eras within a large curvature phase is expected to be of length $n(\log 2)^{-1} \log \eta_{u}$. Combining the results, we find that the expectation value for the length of a large 
curvature phase is given by

$$
\eta_{u}+(\log 2)^{-1}\left(\log \eta_{u}\right)(1-p) \sum_{n=0}^{\infty} n p^{n} \approx \eta_{u}+\eta_{u} \log \eta_{u} \approx \eta_{u} \log \eta_{u}<\infty .
$$

Since the mean length of a large curvature phase is expected to be finite, while the expectation value $\langle L\rangle$ of the length of a small curvature phase is infinite, we conclude that, asymptotically as $l \rightarrow \infty$, small curvature phases dominate over large curvature phases.

Recall that, in the state space description, small curvature phases are phases where the sequence of transitions $\mathcal{S}_{\mathcal{T}}$, which generates the Kasner series $\left(u_{l}\right)_{l \in \mathbb{N}}$ we consider, is captured in a small neighborhood of the Taub points, see figure 5. Accordingly, dominance of small curvature phases over large curvature phases means that the sequence of transitions $\mathcal{S}_{\mathcal{T}}$ spends more and more "time" (as measured by the number of epochs) in a neighborhood of the Taub points as compared to the time spent close to ordinary Kasner states; above all, the ratio diverges in the limit. Considerations of this type quite naturally bring the Taub points into focus when we aim at investigating generic asymptotic dynamics of solutions.

\section{Growth}

In this section, we analyze the analytic and stochastic properties of a function that is naturally associated with a sequence of transitions: the growth function.

\subsection{Auxiliary differential equations and the growth function}

In our analysis, we will encounter certain types of differential equations; the following auxiliary equation represents the common denominator:

$$
\partial_{\tau} A=2\left(1-\Sigma^{2}\right) A
$$

When a solution of (5.6) on the oscillatory subset $\mathcal{O}$ is given, then the equation can be regarded as a linear differential equation with a prescribed time-dependent coefficient $2\left(1-\Sigma^{2}\right)$. Let us now integrate (9.1) along the different Bianchi type I and II transitions. 
Along frame transitions we obtain $A=$ const, since $\left(1-\Sigma^{2}\right)=0$. Single and mixed curvature transitions yield $A\left(\tau_{0}\right) \mapsto A(\tau)=A\left(\tau_{0}\right)\left[\zeta(\tau) / \zeta\left(\tau_{0}\right)\right]$, which follows from (6.3b); note that $\zeta(\tau) / \zeta\left(\tau_{0}\right)>1$ for all $\tau>\tau_{0}$. Letting $\tau_{0} \rightarrow-\infty$ and $\tau \rightarrow \infty$ shows that the initial value of $A$ (i.e., $\lim _{\tau \rightarrow-\infty} A(\tau)$ ) and the final value of $A$ (i.e., $\lim _{\tau \rightarrow \infty} A(\tau)$ ) are related by the growth factor $g$; recall that $g$ is given by

$$
g=\frac{1+u+u^{2}}{1-u+u^{2}}
$$

where $u$ is the (initial) Kasner parameter of the curvature transition; see (6.5). In condensed form, we write

$$
A \mapsto g A
$$

for the effect of a curvature transition on the quantity $A$. Recall that, since $u \in(1, \infty)$ for single and mixed curvature transitions, we have $1<g<3$.

Solving equation (9.1) along a sequence of transitions $\mathcal{S}_{\mathcal{T}}$ on $\mathcal{O}$ amounts to iterating (9.2), which we will elaborate on in the following. (Recall that, in our convention, $\mathcal{S}_{\mathcal{T}}$ contains only Bianchi type I and II transitions, unless otherwise stated.) For the lth curvature transition of a sequence $\mathcal{S}_{\mathcal{T}}$, the growth factor is given by

$$
g_{l}:=\frac{1+u_{l}+u_{l}^{2}}{1-u_{l}+u_{l}^{2}}=\frac{1+u_{l}+u_{l}^{2}}{1+u_{l+1}+u_{l+1}^{2}} r_{l}^{2}, \quad \text { where } r_{l}:=\left(\min \left\{u_{l}-1,1\right\}\right)^{-1}
$$

the quantity $r_{l}$ satisfies $r_{l} \geq 1$, where $r_{l}=1$ whenever $u_{l}>2-$ this is the case when $u_{l+1}=u_{l}-1$, see (6.4). We define the growth function as the product of the growth factors: we let $G: \mathbb{N} \rightarrow \mathbb{R}$ be defined by $G(0)=G_{0}=$ 1 and

$$
G(l)=G_{l}=\prod_{j=0}^{l-1} g_{j}=\prod_{j=0}^{l-1}\left(\frac{1+u_{j}+u_{j}^{2}}{1+u_{j+1}+u_{j+1}^{2}}\right) r_{j}^{2}=\left(\frac{1+u_{0}+u_{0}^{2}}{1+u_{l}+u_{l}^{2}}\right) \prod_{j=0}^{l-1} r_{j}^{2} .
$$

Since $g_{j}>1, r_{j} \geq 1$ for all $j>1$ (and $g_{j} \not \rightarrow 1$ as $j \rightarrow \infty$ ), $G_{l}$ increases rapidly with $l$ and $G_{l} \rightarrow \infty$ with $l \rightarrow \infty$. In our convention, $G_{l}$ denotes the value of the growth function at the beginning of the $l$ th curvature transition; see figure 6 . 


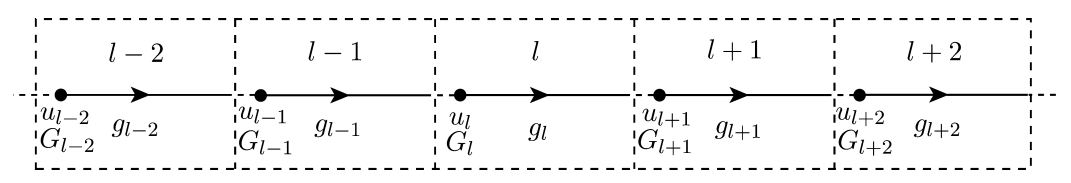

Figure 6: Transition counting conventions.

Iterating (9.2) along $l$ curvature transitions of the sequence $\mathcal{S}_{\mathcal{T}}$ thus yields

$$
A_{0} \mapsto G(l) A_{0}=G_{l} A_{0}=\left(\prod_{j=0}^{l-1} g_{j}\right) A_{0} ;
$$

hence $A$ is a function whose rate of growth is described by the growth function $G$. Finally, in order to write equation (9.4) in proper notation, let $A_{i}$ denote the value of $A$ at the beginning of the $i$ th transition $\mathcal{T}_{i}$ of the sequence $\mathcal{S}_{\mathcal{T}}=\left(\mathcal{T}_{0}, \mathcal{T}_{1}, \mathcal{T}_{2}, \ldots\right) ;$ then we can write

$$
A_{i}=G_{l(i)} A_{0}
$$

recall that $l(i)=l_{i}$ is the curvature transition index associated with $\mathcal{S}_{\mathcal{T}}$, i.e., the number of curvature transitions among $\left\{\mathcal{T}_{0}, \mathcal{T}_{1}, \ldots, \mathcal{T}_{i}\right\}$, see Section 7 . The use of this notation appears superfluous in the present context, since the function $A$ does not change under frame transitions and it would thus suffice to focus on the curvature transitions $l=0,1,2, \ldots$ of $\mathcal{S}_{\mathcal{T}}$; however, next we will discuss a more general differential equation whose solutions are affected by frame transitions.

Consider the following auxiliary differential equation, which is frame dependent:

$$
\partial_{\tau} B=2\left(1-\Sigma^{2}\right) B+2 R_{3}^{2}-2 R_{1}^{2}
$$

as with (9.1), our aim is to integrate this equation along a sequence of transitions $\mathcal{S}_{\mathcal{T}}$.

For simplicity, let $\mathcal{S}_{\mathcal{T}}=\left(\mathcal{T}_{0}, \mathcal{T}_{1}, \mathcal{T}_{2}, \ldots\right)$ be a sequence of transitions that does not contain $\mathcal{T}_{N_{2}}$ and $\mathcal{T}_{N_{2} R_{2}}$ transitions; hence $N_{2} \equiv 0$ along $\mathcal{S}_{\mathcal{T}}$, so that

$$
\partial_{\tau}\left(\Sigma_{2}-2\right)=2\left(1-\Sigma^{2}\right)\left(\Sigma_{2}-2\right)+2 R_{3}^{2}-2 R_{1}^{2}
$$

holds for each transition $\mathcal{T}_{i}$, which follows from (5.6b) and (5.6j). Consequently, $\left(\Sigma_{2}-2\right)$ is a particular solution of (9.6).

The general solution for $B$ along $\mathcal{S}_{\mathcal{T}}$ can thus be constructed explicitly as the superposition of the particular solution $\left(\Sigma_{2}-2\right)$ and the general solution 
of the homogeneous equation. Since the latter is given by (9.5), we obtain

$$
B_{i}=\left(\Sigma_{2}(i)-2\right)+G_{l(i)}\left[B_{0}-\left(\Sigma_{2}(0)-2\right)\right],
$$

where $\Sigma_{2}(i)$ denotes the value of $\Sigma_{2}$ after $i$ transitions (which, by the convention illustrated in figure 6 , is the value at the beginning of the transition with index $i)$. In the generic case, i.e., $B_{0} \neq\left(\Sigma_{2}(0)-2\right)$, we therefore find

$$
B_{i} \sim \text { const } G_{l(i)} \quad(i \rightarrow \infty)
$$

with const $\neq 0$; in particular, $\left|B_{i}\right| \rightarrow \infty$ as $i \rightarrow \infty$. The result (9.7) is slightly modified when $\mathcal{T}_{N_{2}}$ and $\mathcal{T}_{N_{2} R_{2}}$ transitions are taken into account, see Appendix E; however, the generic behavior of $B$ as described by (9.8) remains unchanged.

In Section 7 we have argued that generically sequences of transitions do not contain double curvature transitions; in accordance with these considerations, we have hence focused our analysis on sequences $\mathcal{S}_{\mathcal{T}}$ of this type. In the remainder of this subsection, however, we discuss non-generic sequences, in particular in view of the growth associated with double curvature transitions.

From Section 6 and Appendix D we recall that the equation $\partial_{\tau}\left(2-\Sigma_{3}\right)=$ $2\left(1-\Sigma^{2}\right)\left(2-\Sigma_{3}\right)$ holds along double curvature transitions (i.e., Bianchi type $\mathrm{VI}_{0}$ orbits) and analogously along Bianchi type $\mathrm{VII}_{0}$ orbits. Consequently, we find that $A(\tau)=A\left(\tau_{0}\right)\left(2-\Sigma_{3}\right)(\tau) /\left(2-\Sigma_{3}\right)\left(\tau_{0}\right)$; the behavior of $B$ is identical, since setting $\left(R_{1}, R_{2}, R_{3}\right)=0$ reduces (9.6) to (9.1). Since the $\alpha$-limit of all orbits is a fixed point with $\Sigma_{3}=2$, the expression $\left(2-\Sigma_{3}\right)(\tau) /\left(2-\Sigma_{3}\right)\left(\tau_{0}\right)$ diverges as $\tau_{0} \rightarrow-\infty$. The growth factor $g$ associated with a Bianchi type $\mathrm{VI}_{0} / \mathrm{VII}_{0}$ orbit can thus be considered as being infinite. It is immediate from these considerations that the growth function is ill-defined along sequences of transitions that contain double curvature transitions; it is natural, however, to consider a "piecewise" growth function: following the remarks of Section 7 we choose to view double curvature transitions as repeated interruptions of a regular Kasner sequence. Thereby the concepts introduced in Section 7 (i.e., eras, small/large curvature phases) are still relevant; the interruptions, however, correspond to two successive eras not being "causally connected" - the initial Kasner parameter $u^{\text {in }}$ of an era that arises from the final state of a $\mathcal{T}_{N_{1} N_{2}}$ transition is not related to the Kasner parameter of the preceding era. This suggests that we treat sequences of transitions that contain double curvature transition as a concatenation of "pieces" of standard Kasner sequences glued together by $\mathcal{T}_{N_{1} N_{2}}$ transitions. Along these fragments the growth function is defined in 
the standard way; however, joining the pieces is not possible, since this is connected with a divergent growth along the joining $\mathcal{T}_{N_{1} N_{2}}$ transition.

In anticipation of our considerations in Section 10, we conclude these remarks by considering orbits that are not exactly of Bianchi type $\mathrm{VI}_{0} / \mathrm{VII}_{0}$, but arbitrarily close (in a higher dimensional state space where the $\alpha-/ \omega$ limits of the $\mathrm{VI}_{0} / \mathrm{VII}_{0}$ orbits are saddles). It is immediate that the growth of $A$ and $B$ along such orbits can be arbitrarily large depending on the degree of the type $\mathrm{VI}_{0} / \mathrm{VII}_{0}$ approximation.

\subsection{Properties of the growth function}

Since the growth function is the key element in the description of the behavior of $A$ and $B$, it is appropriate to study this function in more detail. Let us begin by investigating the properties of the growth function $G(l)$ along a sequence of transitions $\mathcal{S}_{\mathcal{T}}$ with regard to the concepts of small and large curvature phases, and eras.

Consider first a small curvature phase $\left[l_{\text {in }}, l_{\text {out }}\right]$, where we assume $\eta_{u}>2$. For all $l$ the growth factor $g_{l}$ is given by

$$
g_{l}=\frac{1+u_{l}+u_{l}^{2}}{1-u_{l}+u_{l}^{2}}=\frac{1+u_{l}+u_{l}^{2}}{1+u_{l+1}+u_{l+1}^{2}}
$$

see (9.3). The growth function satisfies the recursive relation $G_{l+1}=G_{l} g_{l}$, so that we obtain

$$
\frac{G\left(l_{\mathrm{out}}+1\right)}{G\left(l_{\mathrm{in}}\right)}=\prod_{l=l_{\mathrm{in}}}^{l_{\mathrm{out}}} g_{l}=\frac{1+u^{\mathrm{in}}+\left(u^{\mathrm{in}}\right)^{2}}{1+u_{l_{\mathrm{out}}+1}+\left(u_{l_{\mathrm{out}}+1}\right)^{2}} \approx \frac{1+u^{\mathrm{in}}+\left(u^{\mathrm{in}}\right)^{2}}{1+\eta_{u}+\eta_{u}^{2}} \approx \frac{\left(u^{\mathrm{in}}\right)^{2}}{\eta_{u}^{2}}
$$

where the approximation requires $\eta_{u} \gg 1$. Equation (9.10) describes the growth of $G(l)$ during one small curvature phase.

In a large curvature phase $\left[l_{\mathrm{i}}, l_{\mathrm{f}}\right]$, since $u_{l} \leq \eta_{u}$ for all $l \in\left[l_{\mathrm{i}}, l_{\mathrm{f}}\right]$, we have

$$
g_{l}=\frac{1+u_{l}+u_{l}^{2}}{1-u_{l}+u_{l}^{2}} \geq \frac{1+\eta_{u}+\eta_{u}^{2}}{1-\eta_{u}+\eta_{u}^{2}}=: C_{\mathrm{p}}>1 ; \quad C_{\mathrm{p}} \approx 1+2 \eta_{u}^{-1} \text { if } \eta_{u} \gg 1
$$


Therefore, in a large curvature phase, the growth function $G(l)$ behaves like

$$
G(l+1)=G\left(l_{\mathrm{i}}\right) \prod_{k=l_{\mathrm{i}}}^{l} g_{k}>G\left(l_{\mathrm{i}}\right)\left(C_{\mathrm{p}}\right)^{l+1-l_{\mathrm{i}}}
$$

i.e., we observe exponential growth of $G(l)$ with a given base $C_{\mathrm{p}}$.

Let us now discuss the growth of $G(l)$ in an era $\left[l_{\text {in }}, l_{\text {out }}\right]$. We define the era growth factor $\mathfrak{g}$ as

$$
\mathfrak{g}=\frac{G\left(l_{\text {out }}+1\right)}{G\left(l_{\text {in }}\right)}=\left(\prod_{l=l_{\text {in }}}^{l_{\text {out }}-1} g_{l}\right) g_{l_{\text {out }}}=\frac{1+u^{\text {in }}+\left(u^{\text {in }}\right)^{2}}{1+u^{\text {out }}+\left(u^{\text {out }}\right)^{2}} \frac{1+u^{\text {out }}+\left(\overline{\left.u^{\text {out }}\right)^{2}}\right.}{1-u^{\text {out }}+\left(u^{\text {out }}\right)^{2}},
$$

so that

$$
\mathfrak{g} \geq \frac{1+u^{\mathrm{in}}+\left(u^{\mathrm{in}}\right)^{2}}{3} .
$$

Consider the partition of a sequence into $a=0,1,2, \ldots$ eras. For the era with number $a$, the era growth factor $\mathfrak{g}_{a}$ can be written as

$$
\mathfrak{g}_{a}=\frac{1+u_{a}^{\mathrm{in}}+\left(u_{a}^{\mathrm{in}}\right)^{2}}{1-u_{a}^{\text {out }}+\left(u_{a}^{\text {out }}\right)^{2}}=\left(1+u_{a}^{\mathrm{in}}+\left(u_{a}^{\mathrm{in}}\right)^{2}\right) \frac{\left(u_{a+1}^{\mathrm{in}}\right)^{2}}{1+u_{a+1}^{\text {in }}+\left(u_{a+1}^{\mathrm{in}}\right)^{2}} .
$$

The era growth function $\mathcal{G}$ is defined as the product of era growth factors. From $\mathcal{G}_{a+1}=\mathcal{G}_{a} \mathfrak{g}_{a}$ we obtain

$$
\mathcal{G}(a)=\mathcal{G}_{a}=\mathcal{G}_{0} \prod_{c=0}^{a-1} \mathfrak{g}_{c}=\mathcal{G}_{0}\left(\prod_{c=0}^{a-1}\left(u_{c}^{\mathrm{in}}\right)^{2}\right)\left[\frac{1+u_{0}^{\mathrm{in}}+\left(u_{0}^{\mathrm{in}}\right)^{2}}{\left(u_{0}^{\mathrm{in}}\right)^{2}} \frac{\left(u_{a}^{\mathrm{in}}\right)^{2}}{1+u_{a}^{\mathrm{in}}+\left(u_{a}^{\mathrm{in}}\right)^{2}}\right],
$$

where we set $\mathcal{G}_{0}=1$ by convention. The expression in brackets is a number in the interval $(1 / 3,3)$; when $u_{0}^{\text {in }}$ and $u_{a}^{\text {in }}$ are large the number is close to one.

Let us now compute the expectation values of the growth factors. The era growth factor $\mathfrak{g}$, cf. (9.13), does not possess a finite expectation value. This is because the equations contain $u^{\text {in }}\left(=\varkappa^{-1}\right)$ and $\left(u^{\text {in }}\right)^{2}$ :

$$
\left\langle u^{\mathrm{in}}\right\rangle=\int_{0}^{1} w(\varkappa) \varkappa^{-1} d \varkappa=\infty, \quad\left\langle\left(u^{\mathrm{in}}\right)^{2}\right\rangle=\int_{0}^{1} w(\varkappa) \varkappa^{-2} d \varkappa=\infty \Rightarrow\langle\mathfrak{g}\rangle=\infty,
$$

where we have used the probability density $w(\varkappa)$ from Section 8 . The same result applies for the growth factor describing the growth of $G$ in a small 
curvature phase, cf. (9.10). However, the expectation value of the logarithm of the era growth factor can be estimated straightforwardly:

$$
\langle\log \mathfrak{g}\rangle \geq-\log 3+\int_{0}^{1} w(\varkappa) \log \left(1+\varkappa^{-1}+\varkappa^{-2}\right) d \varkappa \approx 1.77 .
$$

By definition, the logarithm of the era growth function $\mathcal{G}$ is the sum of the logarithms of the era growth factors, therefore we expect $\log \mathcal{G}$ to grow according to

$$
\left\langle\log \mathcal{G}_{a}\right\rangle=\left\langle\sum_{c=0}^{a-1} \log \mathfrak{g}_{c}\right\rangle=a\langle\log \mathfrak{g}\rangle \geq 1.77 a
$$

For further results on growth functions, see Appendix F.

\section{Asymptotic shadowing}

\subsection{Shadowing - asymptotic sequences of orbits}

The subsequent considerations constitute the core of our analysis: we provide evidence that the asymptotic dynamics of solutions of Einstein's equations which exhibit a generic spacelike singularity is represented by sequences of Bianchi type I and type II transitions.

The basic assumption we make is that a solution of Einstein's equations $\boldsymbol{X}\left(t, x^{i}\right)$ that possesses a generic spacelike singularity is asymptotically silent. We further assume that the dynamics of $\boldsymbol{X}\left(t, x^{i}\right)$ along a timeline associated with a generic spatial point $x^{i}$ of such a singularity is asymptotically local. This entails that the asymptotic behavior of the solution is described, with an increasing degree of accuracy, by the dynamics on the $\mathrm{SH}$ part of the silent boundary. The SH silent boundary consists of an infinite number of copies of the finite dimensional SH state space that is spanned by the state vector $\boldsymbol{S}=\left(\Sigma_{\alpha \beta}, A_{\alpha}, N_{\alpha \beta}\right)$, where the spatial coordinates act as the infinite index set. Our assumptions thus lead to the conjecture that the asymptotic behavior of the solution $\boldsymbol{X}(\tau)$ at a generic spatial point $x^{i}$ is governed by the dynamics on the $\mathrm{SH}$ state space.

In Section 5 we have analyzed one structure on the SH silent boundary that is expected to be of fundamental importance: the Kasner circle of equilibrium points $\mathrm{K}^{\bigcirc}$. The local dynamical systems analysis of $\mathrm{K}^{\bigcirc}$ suggests that $E_{\alpha}{ }^{i} \rightarrow 0$ as $\tau \rightarrow \infty$, which indicates intrinsic consistency of our considerations. Furthermore, we are led to the conjecture that there exists a "stable 
part" $\boldsymbol{S}_{\text {stable }}$ of the state vector $\boldsymbol{S}$, whose variables vanish in the asymptotic limit, i.e., $\boldsymbol{S}_{\text {stable }}=\left(N_{12}, N_{23}, N_{31}, A_{\alpha}\right) \rightarrow 0$ as $\tau \rightarrow \infty$. This conjecture is intimately connected with the assumption that the asymptotic evolution of a generic solution is largely dominated by Kasner states, in the sense that the solution spends an increasing amount of $\tau$-time in a small neighborhood of the (non-flat part of) Kasner circle.

Since we expect $\boldsymbol{S}_{\text {stable }} \rightarrow 0$, it is the asymptotic behavior of the remaining "oscillatory variables" $\boldsymbol{S}_{\text {osc }}=\left(\Sigma_{\alpha}, R_{\alpha}, N_{1}, N_{2}\right)$ that represents the essential asymptotic dynamics of the solution $\boldsymbol{X}(\tau)$. Under the above assumptions, the Codazzi constraints (3.8c) imply that

$$
N_{1} R_{2} \rightarrow 0, \quad N_{2} R_{2} \rightarrow 0, \quad\left(N_{1}-N_{2}\right) R_{3} \rightarrow 0
$$

as $\tau \rightarrow \infty$, which is to be compared with $\left(5.6 \mathrm{k}^{\prime}\right)$; in the same limit we obtain that $\boldsymbol{S}_{\text {osc }}$ satisfies the oscillatory dynamical system (5.6a) to (5.6h) and the constraint (5.6j). This suggests that the asymptotic dynamics of $\boldsymbol{S}_{\mathrm{osc}}=$ $\left(\Sigma_{\alpha}, R_{\alpha}, N_{1}, N_{2}\right)$ is governed by the dynamics on the oscillatory subset $\mathcal{O}$, which we have shown to be represented by Bianchi type I, II, $\mathrm{VI}_{0}$ transitions - frame and curvature transitions - and Bianchi type $\mathrm{VII}_{0}$ orbits. In brief, asymptotically, the state vector $\boldsymbol{S}_{\text {osc }}$ of a solution $\boldsymbol{X}$ shadows the flow on $\mathcal{O}$.

Along the orbit $\boldsymbol{S}_{\text {osc }}(\tau)$ the SH Codazzi constraints (5.6k) are satisfied only in the limit $\tau \rightarrow \infty$; hence the disconnectedness of the Bianchi components of the oscillatory subset $\mathcal{O}$ is irrelevant for the solution $\boldsymbol{S}_{\text {osc }}(\tau)$. As an immediate consequence of asymptotic shadowing it thus follows that in the asymptotic regime $\tau \rightarrow \infty$, the orbit $\boldsymbol{S}_{\text {osc }}(\tau)$ resembles a sequence of Bianchi transitions and orbits: $\boldsymbol{S}_{\text {osc }}(\tau)$ can be partitioned into a sequence of segments, where each segment is associated with a heteroclinic orbit on $\mathcal{O}$ (of type I, II, $\mathrm{VI}_{0}$, or $\mathrm{VII}_{0}$ ). Two subsequent segments of $\boldsymbol{S}_{\text {osc }}(\tau)$ are joined smoothly in a neighborhood of an equilibrium point of the Kasner circle. ${ }^{12}$ As $\tau \rightarrow \infty$, the segments of $\boldsymbol{S}_{\text {osc }}(\tau)$ are approximated to an increasing degree of accuracy by heteroclinic orbits on $\mathcal{O}$; simultaneously, the joining of segments occurs increasingly closer to $\mathrm{K}^{\bigcirc}$; hence $\boldsymbol{S}_{\text {osc }}(\tau)$ is described by a sequence of Bianchi transitions and orbits with an increasing degree of accuracy. We may call this representation of the orbit $\boldsymbol{S}_{\text {osc }}(\tau)$ in the asymptotic regime an asymptotic sequence of $\mathcal{O}$-orbits $\mathcal{A S}_{\mathcal{O}}$ (or, for brevity, simply asymptotic sequence). Note that the concept of asymptotic sequences $\mathcal{A S}_{\mathcal{O}}$ gives a precise meaning to BKL's "piecewise approximations".

\footnotetext{
${ }^{12}$ The partition of $\boldsymbol{S}_{\text {osc }}(\tau)$ into segments involves a certain arbitrariness: which point in the neighborhood of $\mathrm{K}^{\bigcirc}$ is chosen to be defined as the end point of one transition and the initial point of the subsequent one is a matter of convention; however, asymptotically any reasonable convention leads to the natural segmentation into the heteroclinic orbits on $\mathcal{O}$.
} 
As it will turn out in the following, generic asymptotic sequences $\mathcal{A S}_{\mathcal{O}}$ fall into segments that correspond to Bianchi type I and II transitions; it is suggestive to call asymptotic sequences of this kind asymptotic sequences of transitions $\mathcal{A S}_{\mathcal{T}}$. With slight abuse of notation we write $\mathcal{A S}_{\mathcal{T}}=\left(\mathcal{T}_{i}\right)_{i \in \mathbb{N}}$, where $\mathcal{T}_{i}$ does not denote an exact transition in present context, but a segment of $\mathcal{A S}_{\mathcal{O}}$, which we can view as an approximate transition.

However, a priori we cannot exclude that Bianchi type $\mathrm{VI}_{0}$ transitions (double curvature transitions) and Bianchi type $\mathrm{VII}_{0}$ orbits play an important role in the context of asymptotic sequences $\mathcal{A S}_{\mathcal{O}}$. A segment of $\mathcal{A S}_{\mathcal{O}}$ that is approximated by a type $\mathrm{VI}_{0} / \mathrm{VII}_{0}$ orbit - an approximate type $\mathrm{VI}_{0} / \mathrm{VII}_{0}$ orbit in our nomenclature - is expected to connect a neighborhood of the point $\mathrm{T}_{3}$ with a neighborhood of one of the fixed points on sectors (312) or (321) on $\mathrm{K}^{\bigcirc}$. Clearly, since the accuracy of the approximation, i.e., the degree of shadowing, improves as $\tau \rightarrow \infty$, these neighborhoods are expected to shrink with increasing $\tau$. However, this does not automatically lead to an exclusion of type $\mathrm{VI}_{0} / \mathrm{VII}_{0}$ orbits from generic asymptotic sequences. Instead, the exclusion of type $\mathrm{VI}_{0} / \mathrm{VII}_{0}$ orbits follows from a bootstrap-like argument which we present next.

\section{$10.2 \quad R_{2} \rightarrow 0$ and $N_{2} \rightarrow 0$ as $\tau \rightarrow \infty$}

Consider an asymptotic sequence $\mathcal{A S}_{\mathcal{O}}$. Equations (5.6a) to (5.6h) imply that the product $\left(R_{1} R_{3}\right)$ satisfies

$$
\partial_{\tau}\left(R_{1} R_{3}\right)=4\left(1-\Sigma^{2}\right) R_{1} R_{3}+\left(\Sigma_{1}-\Sigma_{3}\right) R_{1} R_{3}+2\left(R_{3}^{2}-R_{1}^{2}\right) R_{2}
$$

with increasing accuracy as $\tau \rightarrow \infty$. Using the auxiliary quantity $B$, which is subject to the auxiliary equation (9.6), we obtain an identical equation for $\left(R_{2} B\right)$, i.e.,

$$
\partial_{\tau}\left(R_{2} B\right)=4\left(1-\Sigma^{2}\right) R_{2} B+\left(\Sigma_{1}-\Sigma_{3}\right) R_{2} B+2\left(R_{3}^{2}-R_{1}^{2}\right) R_{2} .
$$

By choosing appropriate initial data for $B$, it follows that $\left(R_{2} B\right)$ coincides with $\left(R_{1} R_{3}\right)$. Accordingly we obtain

$$
R_{2} B=R_{1} R_{3} \quad \text { or } \quad\left|R_{2}\right| \sim\left|R_{1} R_{3}\right| B^{-1} .
$$

Generically, along an asymptotic sequence of transitions $\mathcal{A S}_{\mathcal{T}}$, the quantity $B$ satisfies $B_{i} \sim$ const $G_{l(i)}$ in the asymptotic regime $i \rightarrow \infty$, where $G_{l}$ is the growth function of Section 9; this is because $\mathcal{A S}_{\mathcal{T}}$ is approximated 
by a sequence of transitions $\mathcal{S}_{\mathcal{T}}$ in the limit $\tau \rightarrow \infty$. Along an asymptotic sequence $\mathcal{A S}_{\mathcal{O}}$ (which, in contrast to $\mathcal{A} \mathcal{S}_{\mathcal{T}}$, might also contain type $\mathrm{VI}_{0} / \mathrm{VII}_{0}$ orbits), the behavior of $B$ described by the growth function holds at least piecewise, where the interruptions are associated with the approximate type $\mathrm{VI}_{0} / \mathrm{VII}_{0}$ orbits of $\mathcal{A} \mathcal{S}_{\mathcal{O}}$; when the solution shadows a Bianchi type $\mathrm{VI}_{0} / \mathrm{VII}_{0}$ orbit, a large increase in $B$ ensues, see Section 9 . Since type $\mathrm{VI}_{0} / \mathrm{VII}_{0}$ orbits thus strengthen the growth of $B$ in relation to the normal growth described by $G_{l(i)}$, we conclude that the relation $B_{i} \sim \operatorname{const} G_{l(i)}$ can be regarded as representing a lower bound for the growth of $B$.

We can therefore write (10.2) as $\left|R_{2}\right|_{i} \sim$ const $\left|R_{1} R_{3}\right|_{i} G_{l(i)}^{-1}$ as $i \rightarrow \infty$, or in simplified notation,

$$
\left|R_{2}\right| \sim \text { const }\left|R_{1} R_{3}\right| G^{-1}
$$

Since $\left|R_{1} R_{3}\right|$ is bounded because of the Gauss constraint (5.6j), equation (10.3) implies that $\left|R_{2}\right| \rightarrow 0$ as $\tau \rightarrow \infty$, i.e., generically $R_{2}$ is asymptotically suppressed. Analogously, $\left|N_{2}\right| \rightarrow 0$ as $\tau \rightarrow \infty$, which we show in the following.

Consider the product $\left(N_{1} R_{3}^{2}\right)$; from $(5.6 \mathrm{a})$ to $(5.6 \mathrm{~h})$ we have

$$
\partial_{\tau}\left(N_{1} R_{3}^{2}\right)=2\left[2\left(1-\Sigma^{2}\right)-\Sigma^{2}-\Sigma_{2}\right] N_{1} R_{3}^{2}-4 R_{1} R_{2} R_{3} N_{1} .
$$

Since, generically, $R_{2} \sim$ const $\left(R_{1} R_{3}\right) G^{-1}$ by (10.3), we obtain

$$
\partial_{\tau}\left(N_{1} R_{3}^{2}\right)=2\left[2\left(1-\Sigma^{2}\right)-\Sigma^{2}-\Sigma_{2}\right] N_{1} R_{3}^{2}-4 R_{1}^{2} G^{-1}\left(N_{1} R_{3}^{2}\right) .
$$

In the asymptotic regime $\tau \rightarrow \infty$ the last term in (10.4) vanishes; in fact, by using the results of the previous sections we show in Appendix $F$ that the term falls off rapidly enough so that

$$
\partial_{\tau}\left(N_{1} R_{3}^{2}\right)=2\left[2\left(1-\Sigma^{2}\right)-\Sigma^{2}-\Sigma_{2}\right] N_{1} R_{3}^{2}
$$

holds asymptotically. Similarly, using the auxiliary quantity $A$ leads to the equation

$$
\partial_{\tau}\left(N_{2} A^{2}\right)=2\left[2\left(1-\Sigma^{2}\right)-\Sigma^{2}-\Sigma_{2}\right] N_{2} A^{2}
$$

for $\left(N_{2} A^{2}\right)$. By choosing appropriate initial data for $A$, we conclude that

$$
\left|N_{2} A^{2}\right| \sim \text { const }\left|N_{1} R_{3}^{2}\right| ;
$$


equivalently, by using (9.5), we can write $\left|N_{2}\right|_{i} \sim$ const $\left|N_{1} R_{3}^{2}\right|_{i} G_{l(i)}^{-2}$, or simply,

$$
\left|N_{2}\right| \sim \text { const }\left|N_{1} R_{3}^{2}\right| G^{-2} .
$$

Since $\left|R_{3}^{2}\right|$ and $\left|N_{1}-N_{2}\right|$ are bounded because of (5.6j), equation (10.6) implies that, generically, $\left|N_{2}\right| \rightarrow 0$ as $\tau \rightarrow \infty$.

\subsection{Exclusion of $R_{2}$ and $N_{2}$}

Here we establish the following result: the statement $\left(R_{2}, N_{2}\right) \rightarrow 0(\tau \rightarrow \infty)$ does not merely mean that the amplitudes in $R_{2}$ and $N_{2}$ are decreasing, but it also leads to a complete or stochastic exclusion of the shadowing of orbits that involve $R_{2}$ or $N_{2}$, i.e., $\mathcal{T}_{R_{2}}, \mathcal{T}_{N_{2}}, \mathcal{T}_{R_{2} N_{2}}, \mathcal{T}_{R_{1} R_{2} R_{3}}, \mathcal{T}_{N_{1} N_{2}}$ transitions and Bianchi type $\mathrm{VII}_{0}$ orbits.

In Section 6 and Appendix D we have proved that there are no Bianchi type $\mathrm{VI}_{0}\left(\mathcal{T}_{N_{1} N_{2}}\right)$ and $\mathrm{VII}_{0}$ orbits with arbitrarily small amplitudes: there exists $\epsilon>0$ such that $\max \left|N_{2}\right| \geq \epsilon$ uniformly for all type $\mathrm{VI}_{0} / \mathrm{VII}_{0}$ orbits. Since, generically, $\left|N_{2}\right| \rightarrow 0$ along asymptotic sequences $\mathcal{A S}_{\mathcal{O}}$, this entails that Bianchi type $\mathrm{VI}_{0}$ and $\mathrm{VII}_{0}$ orbits cannot be contained in generic sequences $\mathcal{A S}_{\mathcal{O}}$ in the asymptotic regime, as for sufficiently large $\tau,\left|N_{2}\right|$ satisfies $\left|N_{2}\right|<\epsilon$. Since the shadowing of Bianchi type $\mathrm{VI}_{0}$ and $\mathrm{VII}_{0}$ orbits is thus completely excluded, asymptotic sequences $\mathcal{A S}_{\mathcal{O}}$ are sequences of transitions $\mathcal{A S}_{\mathcal{T}}$ which consist of Bianchi type I/II transitions only. In brief, a generic $\mathcal{A S}_{\mathcal{O}}$ must be an $\mathcal{A S}_{\mathcal{T}}$.

Let us now consider the remaining transitions that involve the excitation of one of the variables $R_{2}$ or $N_{2}$, i.e., $\mathcal{T}_{R_{2}}, \mathcal{T}_{N_{2}}, \mathcal{T}_{R_{2} N_{2}}, \mathcal{T}_{R_{1} R_{2} R_{3}}$ transitions. Consider, for instance, the case of $\mathcal{T}_{R_{2}}$ transitions: for a given $\mathcal{T}_{R_{2}}$ transition, the quantity $\left|R_{2}\right|$ goes through a finite maximum $r$ along the transition orbit, which can be computed via the Gauss constraint (5.6j). However, since $\left|R_{2}\right| \rightarrow 0$ as $\tau \rightarrow \infty$ by (10.3), $\left|R_{2}\right|$ can never reach the value $r$, and hence the given transition cannot take place when $\tau$ is sufficiently large.

It follows that more and more $\mathcal{T}_{R_{2}}$ transitions are excluded as $\tau \rightarrow \infty$, so that merely transitions in increasingly smaller neighborhoods of $\mathrm{T}_{2}$ and $\mathrm{Q}_{2}$ are possible; however, it is a priori not clear whether these transitions "die out" completely after some time, or if there are infinitely many as $\tau \rightarrow \infty$. Consider, e.g., a neighborhood of the point $\mathrm{T}_{2}$ : the repeated occurrence of alternating sequences of $\mathcal{T}_{N_{1}}$ and $\mathcal{T}_{R_{2}}$ transitions (generating small curvature phases) is quite plausible a priori, particularly in the light of the statement that small curvature phases dominate in sequences of transitions $\mathcal{S}_{\mathcal{T}}$ and 
thus in the asymptotic dynamics of $\mathcal{A S}_{\mathcal{T}}$; see the discussion in Section 8 . However, in the following we establish a proposition that implies that $\mathcal{T}_{R_{2}}$ (and $\mathcal{T}_{N_{2}}$ ) transitions are excluded stochastically for generic sequences $\mathcal{A S}_{\mathcal{T}}$ :

Proposition (Stochastic exclusion of $R_{2}$ and $N_{2}$ ). A generic asymptotic sequence $\mathcal{A S}_{\mathcal{T}}$ contains a finite number of $\mathcal{T}_{R_{2}}, \mathcal{T}_{R_{1} R_{2} R_{3}}, \mathcal{T}_{N_{2}}$, and $\mathcal{T}_{N_{2} R_{2}}$ transitions only.

As for the previous results, the word "generic" is important also in this context; there might exist sequences $\mathcal{A S}_{\mathcal{T}}$ that contain an infinite number of transitions that involve $R_{2}$ or $N_{2}$; however, these sequences form a set of measure zero in the space of all sequences. Recall further that asymptotic sequences of transitions $\mathcal{A S}_{\mathcal{T}}$ are the generic case of asymptotic sequences $\mathcal{A S}_{\mathcal{O}}$; hence, in the proposition it is equivalent to write $\mathcal{A S}_{\mathcal{O}}$ instead of $\mathcal{A} \mathcal{S}_{\mathcal{T}}$.

The validity of the proposition relies on stochastic arguments, which we present in the remainder of this subsection. For simplicity we focus on the statement that there is a finite number of $\mathcal{T}_{R_{2}}$ transitions in a generic asymptotic sequence $\mathcal{A S}_{\mathcal{T}}$. The treatment of the other transitions is analogous.

Consider an arbitrary $\mathcal{T}_{R_{2}}$ frame transition. Along the $\mathcal{T}_{R_{2}}$ orbit the quantity $\left|R_{2}\right|$ goes through a maximum $r$ that is determined by the Gauss constraint (5.6j); it is straightforward to express $r$ in terms of the Kasner parameter $u$ that characterizes the $\mathcal{T}_{R_{2}}$ transition: in particular, if $u \gg 1$ (which corresponds to a $\mathcal{T}_{R_{2}}$ transition that takes place in a small neighborhood of $\left.\mathrm{T}_{2}\right)$, we obtain $r \sim 3 u^{-1}$; if $(u-1) \ll 1$, then the corresponding $\mathcal{T}_{R_{2}}$ transition is close to $\mathrm{Q}_{2}$ and $r \sim(u-1)$; cf. figure 3 .

By equation (10.3), along $\mathcal{A S}_{\mathcal{T}}$ the quantity $\left|R_{2}\right|$ decreases with time at a rate given by $\left|R_{2}\right|_{l(i)} \propto G_{l}^{-1}$; hence, a necessary condition for an (approximate) $\mathcal{T}_{R_{2}}$ transition to take place after the $l$ th (approximate) curvature transition of $\mathcal{A S}_{\mathcal{T}}$ is that $u$ be sufficiently large (or $(u-1)$ be sufficiently small):

An occurrence of a $\mathcal{T}_{R_{2}}$ transition close to $\left\{\begin{array}{l}\mathrm{T}_{2} \\ \mathrm{Q}_{2}\end{array}\right\}$ is only possible if

$$
\left\{\begin{array}{c}
u_{l}^{-1}<c G_{l}^{-1} \\
u_{l}-1<c G_{l}^{-1}
\end{array}\right\}
$$

where $c$ is a constant. Based on these considerations, we find that establishing the proposition amounts to proving that for generic sequences the conditions in (10.7) are satisfied only for finitely many $l$ as $l \rightarrow \infty$. For simplicity, we focus on the first case of (10.7), i.e., we investigate the condition $u_{l}^{-1}<c G_{l}^{-1}$; it is straightforward to adapt the results to the second case. 
Since asymptotic sequences $\mathcal{A S}_{\mathcal{T}}$ shadow sequences of transitions $\mathcal{S}_{\mathcal{T}}$, we can adopt the concepts introduced in Section 7; in particular, to obtain a more transparent formulation of the problem, we make use of the concept of eras: we view $\mathcal{A S}_{\mathcal{T}}$ as a succession of eras $\left[\left(l_{\text {in }}\right)_{a},\left(l_{\text {out }}\right)_{a}\right](a=0,1,2,3, \ldots)$, and denote by $\varkappa_{a} \in(0,1)$ the value of $u^{-1}$ at the beginning of era $a$, i.e., $\varkappa_{a}=\left(u_{a}^{\text {in }}\right)^{-1}$. Furthermore, we define $\delta_{a}$ to be the value of the function on the r.h.s. of condition (10.7) at the beginning of era $a$, i.e.,

$$
\delta_{a}=c G_{\left(l_{\text {in }}\right)_{a}}^{-1}=c G_{\left(l_{\text {out }}+1\right)_{a-1}}^{-1}=c \mathcal{G}_{a}^{-1},
$$

where $\mathcal{G}_{a}$ is the era growth function defined in Section 9. Without loss of generality, we assume the initial value $\delta_{0}$ of the sequence to be sufficiently small, i.e., $\delta_{0}<\varepsilon$. Based on these concepts, the first case of (10.7) translates to the following necessary condition for the occurrence of $\mathcal{T}_{R_{2}}$ transitions:

An occurrence of a $\mathcal{T}_{R_{2}}$ transition close to $\mathrm{T}_{2}$ in era $a$ is only possible if

$$
\varkappa_{a}<\delta_{a} \text {. }
$$

To establish the proposition we show that for generic sequences the condition $\varkappa_{a}<\delta_{a}$ is satisfied for a finite number of eras only.

The arguments we present in the following are stochastic in nature. Our basic assumption is that we can model the sequence $\left(\varkappa_{a}\right)_{a \in \mathbb{N}}$ by a random variable $\varkappa$ on $(0,1)$ with a well-defined probability density. The justification for this assumption and its origin are discussed in Section 10.4 below. For the subsequent computations, we employ the density $w(\varkappa)$ that describes the stochastics of Kasner sequences; however, the basic qualitative results are largely independent of the concrete form of the probability density, see the remarks at the end of this subsection.

Since $\varkappa$ is a random variable, the sequence $\left(\varkappa_{a}\right)_{a \in \mathbb{N}}$ represents an infinite series of trials with $\varkappa$. The sequence $\left(\delta_{a}\right)_{a \in \mathbb{N}}$, on the other hand, represents a sequence $\left(0, \delta_{a}\right)$ of intervals whose length decreases as $a \rightarrow \infty$. The condition (10.8) thus reads: the number of eras that can contain $\mathcal{T}_{R_{2}}$ transitions coincides with the number of "hits" $\varkappa_{a} \in\left(0, \delta_{a}\right)$ as $a \rightarrow \infty$.

In Appendix $\mathrm{G}$ we demonstrate that the behavior of the sequence $\left(\delta_{a}\right)_{a \in \mathbb{N}}$ is decisive for the "hit rate": $\delta_{a} \sim a^{-1}$ leads to a diverging expectation value for the number of hits as $a \rightarrow \infty$. In our case, this would lead to the conclusion that an infinite number of eras can contain $\mathcal{T}_{R_{2}}$ transitions (although the recurrence intervals between $\mathcal{T}_{R_{2}}$ transitions would become increasingly large). A sequence $\delta_{a} \sim a^{-2}$, on the other hand, generates a finite expectation value; in that case only a finite number of eras is expected to contain $\mathcal{T}_{R_{2}}$ transitions. 
The present situation is more complicated since $\left(\delta_{a}\right)_{a \in \mathbb{N}}$ is not given explicitly, but is instead determined by a stochastic process. We have the relation

$$
\delta_{a+1}=c \mathcal{G}_{a+1}^{-1}=c \mathcal{G}_{a}^{-1} \mathfrak{g}_{a}^{-1}=\mathfrak{g}_{a}^{-1} \delta_{a},
$$

see Section 9, and by using (9.13) and (9.14),

$$
\delta_{a+1}<\frac{3}{1+u_{a}^{\mathrm{in}}+\left(u_{a}^{\mathrm{in}}\right)^{2}} \delta_{a}=\frac{3 \varkappa_{a}^{2}}{1+\varkappa_{a}+\varkappa_{a}^{2}} \delta_{a} .
$$

Equivalently we can write

$$
\log \delta_{a+1}-\log \delta_{a}<\log 3+\log \varkappa_{a}^{2}-\log \left(1+\varkappa_{a}+\varkappa_{a}^{2}\right) .
$$

Employing the probability density $w(\varkappa)$ for $\varkappa$, see (8.4), we find an estimate for the expectation value of $\log \delta_{a+1} / \delta_{a}$,

$$
\begin{aligned}
& \left\langle\log \delta_{a+1}-\log \delta_{a}\right\rangle<\log 3+\int_{0}^{1} w(\varkappa)\left(\log \varkappa^{2}-\log \left(1+\varkappa+\varkappa^{2}\right)\right) d \varkappa \\
& \quad \approx-1.77<-1
\end{aligned}
$$

see also (9.15). Accordingly, for $a \gg 1$, we expect

$$
\left\langle\log \delta_{a}\right\rangle<-a+\log \delta_{0}<-a+\log \varepsilon .
$$

Consequently, the condition (10.8), i.e., $\varkappa_{a}<\delta_{a}$ (or, equivalently, $\log \varkappa_{a}<$ $\log \delta_{a}$ ) translates to the following statement:

An occurrence of a $\mathcal{T}_{R_{2}}$ transition close to $\mathrm{T}_{2}$ in era $a$ is only possible if

$$
\varkappa_{a}<\varepsilon e^{-a} \text {. }
$$

Based on these considerations, we can compute the probabilities for " $N$ hits" (cf. Appendix G). For $a \gg 1$, the probability that $\varkappa \in\left(0, \varepsilon e^{-a}\right)$ is given by

$$
\mathrm{P}\left(\varkappa \in\left(0, \varepsilon e^{-a}\right)\right)=\int_{0}^{\varepsilon e^{-a}} w(\varkappa) d \varkappa=: \tilde{\delta}_{a} \approx \varepsilon w(0) e^{-a} .
$$


For a series of $A$ trials, we find that the probability $P^{A}(0)$ ("no hit", i.e., the probability for the scenario $\left.\varkappa \notin\left[0, \varepsilon e^{-a}\right] \forall a=1, \ldots, A\right)$ is given by

$$
\begin{aligned}
\log \mathrm{P}^{A}(0) & =\sum_{a=1}^{A} \log \left(1-\tilde{\delta}_{a}\right) \approx \sum_{a=1}^{A}\left(-\varepsilon w(0) e^{-a}+O\left(\varepsilon^{2} e^{-2 a}\right)\right) \\
& \approx-\varepsilon w(0) \frac{1-e^{-A}}{1-e^{-1}}
\end{aligned}
$$

Similarly, the probability for "one hit" is

$$
\begin{aligned}
P^{A}(1) & =P^{A}(0) \sum_{a=1}^{A} \frac{\tilde{\delta}_{a}}{1-\tilde{\delta}_{a}} \approx P^{A}(0)\left[\varepsilon w(0) \sum_{a=1}^{A} e^{-a}\right] \\
& \approx P^{A}(0)\left[\varepsilon w(0) \frac{1-e^{-A}}{1-e^{-1}}\right],
\end{aligned}
$$

and the probability for "two hits" in a series of $A$ trials, $\mathrm{P}^{A}(2)$, is given by

$$
\begin{aligned}
\frac{P^{A}(2)}{P^{A}(0)} & =\sum_{a<b} \frac{\tilde{\delta}_{a}}{1-\tilde{\delta}_{a}} \frac{\tilde{\delta}_{b}}{1-\tilde{\delta}_{b}} \approx \frac{1}{2}\left[\left(\sum_{a} \frac{\tilde{\delta}_{a}}{1-\tilde{\delta}_{a}}\right)^{2}-\sum_{a}\left(\frac{\tilde{\delta}_{a}}{1-\tilde{\delta}_{a}}\right)^{2}\right] \\
& <\frac{1}{2}\left[\varepsilon w(0) \frac{1-e^{-A}}{1-e^{-1}}\right]^{2} .
\end{aligned}
$$

Letting $A \rightarrow \infty$, we obtain

$$
P^{\infty}(0) \approx e^{-p} \approx 1-p, \quad P^{\infty}(1)<p, \quad P^{\infty}(2)<\frac{p^{2}}{2}, \ldots,
$$

where $p=\varepsilon w(0)\left(1-e^{-1}\right)^{-1}$. We conclude that the expectation value of the number of hits is finite:

$$
\langle \# \text { hits }\rangle=\sum_{i=1}^{\infty} i P^{\infty}(i)<\sum_{i=1}^{\infty} i \frac{p^{i}}{i !}=p e^{p}<\infty .
$$

Since the "number of hits" corresponds to the number of eras that contain $\mathcal{T}_{R_{2}}$ transitions, we have shown that the number of eras that contain $\mathcal{T}_{R_{2}}$ transitions is expected to be finite and thus we have established the proposition. 
It is important to stress that the arguments of the proof rely on the assumption that the sequence $\mathcal{A S}_{\mathcal{T}}$ is generic. By stochastic methods, it is impossible to exclude that there exist (non-generic) sequences that contain an infinite number of $\mathcal{T}_{R_{2}}$ (or $\mathcal{T}_{N_{2}}, \mathcal{T}_{N_{2} R_{2}}, \mathcal{T}_{R_{1} R_{2} R_{3}}$ ) transitions.

We conclude this subsection with the observation that the above results are to a large extent independent of the concrete form of the probability density $w(\varkappa)$; e.g., it is sufficient to assume that $w(0)$ is finite to obtain the result (10.9). This is essentially due to the rapid decrease of $\delta_{a}$, which is an exponential decay that stems from the (era) growth function.

Let us finally summarize the results of this subsection: Bianchi type $\mathrm{VI}_{0}$ and $\mathrm{VII}_{0}$ orbits are excluded from generic asymptotic sequences $\mathcal{A S}_{\mathcal{O}}$; therefore, a generic asymptotic sequence $\mathcal{A S}_{\mathcal{O}}$ is an asymptotic sequence of transitions $\mathcal{A S}_{\mathcal{T}}$. Generically sequences $\mathcal{A S}_{\mathcal{T}}$ do not contain transitions that involve an excitation of the variables $R_{2}$ and $N_{2}$, i.e., the transitions $\mathcal{T}_{\mathrm{N}_{2} R_{2}}$, $\mathcal{T}_{R_{2}}, \mathcal{T}_{N_{2}}$, and $\mathcal{T}_{R_{1} R_{2} R_{3}}$ do not appear. The arguments leading to the stochastic exclusion of $R_{2}$ and $N_{2}$ are based on the stochastic properties of asymptotic sequences; next we discuss the stochasticity of asymptotic sequences in more detail.

\subsection{Randomized Kasner sequences}

In Section 7 we have seen that generic sequences of transitions $\mathcal{S}_{\mathcal{T}}$ are associated with Kasner sequences $\left(u_{l}\right)_{l \in \mathbb{N}}$, which are determined by the recursion (7.1), where the initial value $u_{0} \notin \mathbb{Q}$. In Section 8 we have presented a stochastic analysis of Kasner sequences: the collective distribution of all sequences $\left(u_{j}^{\text {in }}\right)_{j \in \mathbb{N}}$ (or, equivalently, $\left.\left(\varkappa_{j}\right)_{j \in \mathbb{N}}=\left(u_{j}^{\text {in }}\right)_{j \in \mathbb{N}}^{-1}\right)$ possesses a probabilistic description in terms of a probability density $w$. In the context of asymptotic sequences of transitions $\mathcal{A S}_{\mathcal{T}}$, however, we do not deal with exact Kasner sequences; in the following we investigate the resulting consequences for the probabilistic aspects.

An asymptotic sequence of transitions $\mathcal{A S}_{\mathcal{T}}$ generates an approximate Kasner map, i.e.,

$$
u_{l+1} \approx \begin{cases}u_{l}-1 & \text { if } u_{l}>2, \\ \left(u_{l}-1\right)^{-1} & \text { if } 1<u_{l}<2 .\end{cases}
$$

Since asymptotic sequences $\mathcal{A S}_{\mathcal{T}}$ shadow sequences $\mathcal{S}_{\mathcal{T}}$ to an increasing degree of accuracy, the errors in (10.10) converge to zero as $\tau \rightarrow \infty$. We presume that the nature of these errors is random, which is due to the fact 
that the approach $\mathcal{A S}_{\mathcal{T}} \rightarrow \mathcal{S}_{\mathcal{T}}$ is effectively unpredictable. This random element in the approach $\mathcal{A S}_{\mathcal{T}} \rightarrow \mathcal{S}_{\mathcal{T}}$ is the key to a probabilistic description of an approximate Kasner sequence (10.10): while an exact Kasner sequence might not be described by the collective probability density $w(\varkappa)$ (or alternatively $\hat{w}(\varkappa)$ ), the random errors in (10.10) smear out the probability distributions of exact sequences over the entire collective; we thus expect the sequence $\left(\varkappa_{j}\right)_{j \in \mathbb{N}}$ associated with an individual generic randomized asymptotic Kasner sequence to be described by the probability density $w(\varkappa)$ of Section 8. In brief, we can regard (10.10) as a randomized asymptotic Kasner sequence which admits a probabilistic description in terms of the density $w(\varkappa)$.

The errors in (10.10) are not uniform in $u$ (i.e., independent of the value of $u$ ): the Kasner map $u \mapsto u-1$ (or $u \mapsto(u-1)^{-1}$ ) is relatively robust when $u$ takes a value that is far from the extremes (which are $u=1$ and $u=\infty)$, i.e., when $u \in\left(1+\varepsilon, \varepsilon^{-1}\right)$ for some $\varepsilon$; in contrast, the errors in the Kasner map might be large when $u^{-1}$ or $(u-1)$ is small. To see this, we consider the (approximate) curvature transitions that generate (10.10). When $u \in\left(1+\varepsilon, \varepsilon^{-1}\right)$, the associated curvature transition connects two points on the Kasner circle $\mathrm{K}^{\bigcirc}$ where the (angular) gradient of $u$ (i.e., the gradient of $u$ along the Kasner circle) is comparatively small, see figure 1(b). A perturbation of the curvature transition, i.e., a deviation from the exact orbit, thus leads to a small error in the Kasner map. In contrast, when $u>\varepsilon^{-1}$, the associated curvature transition takes place in a small neighborhood of one of the Taub points, where the angular gradient of $u$ is large, see figure 1(b); hence, any deviation from the exact curvature transition orbit leads to a large error in the $u$-map. This effect is amplified by the local characteristics in the approach $\mathcal{A S}_{\mathcal{T}} \rightarrow \mathcal{S}_{\mathcal{T}}$ : we expect the accuracy of the shadowing to be smaller in the neighborhood of the Taub points (and the points $\mathrm{Q}_{\alpha}$ ) than elsewhere. The dynamics in the neighborhood of the Taub points is rather complicated, which is due to the fact that these fixed points are not transversally hyperbolic: the approach to the attractor is expected to be rather slow, and for fixed points close to the Taub points, the actual dynamics is expected to display relatively large deviations from the linearized dynamics (more accurately described by "eras of small oscillations"). It is evident that this leads to an additional blow-up of errors in the $u$-map for large $u$.

Based on these consideration, we expect that the Kasner map is not well-defined unless $u \in\left(1+\varepsilon, \varepsilon^{-1}\right)$; only for this "safe range" of $u$ we can assume that (10.10) holds. However, in the asymptotic regime the safe range increases, i.e., $\varepsilon \rightarrow 0$ as $\tau \rightarrow \infty$, which is simply because $\mathcal{A S}_{\mathcal{T}} \rightarrow \mathcal{S}_{\mathcal{T}}$. Suppose that the safe range grows rapidly enough. Then we can draw the 
following conclusion: the probability that the sequence $\left(u_{l}\right)_{l \in \mathbb{R}}$ ever leaves the safe range is zero. To see this, we use the same arguments that led to the stochastic exclusion of $R_{2}$ and $N_{2}$ : the "number of hits" $\varkappa_{j} \in\left(0, \epsilon_{j}\right)$ (corresponding to the Kasner sequence leaving the safe range) has a finite expectation value.

It is plausible that the increase of the safe range is in fact an exponential growth, since it is directly related to the accuracy of the shadowing. The decay rates of $R_{2}$ and $N_{2}$ suggest that the overall accuracy of the shadowing behaves in the same way, which in turn leads to the required rapid growth of the safe range. Presuming the correctness of these considerations, we find that generic asymptotic Kasner sequences are indeed described by (10.10), where the errors converge to zero (uniformly) as $\tau \rightarrow \infty$.

\section{The billiard attractor}

In the previous section we have argued that for generic solutions the variables $R_{2}$ and $N_{2}$ are not excited in the asymptotic regime, i.e., we have established the exclusion of transitions that involve the variables $R_{2}$ and $N_{2}$ from generic asymptotic sequences of transitions. Accordingly, the essential asymptotic dynamics of generic solutions $\boldsymbol{X}(\tau)$ is described not by the full oscillatory dynamical system (5.6a) to (5.6h), but instead by a reduced system that is obtained by setting $R_{2}=0$ and $N_{2}=0$.

\subsection{The billiard subset}

We define the billiard subset $\mathcal{O}_{\mathcal{B}}$ as the invariant subset $\left(R_{2}=0\right) \wedge\left(N_{2}=0\right)$ of the oscillatory subset $\mathcal{O}$, which leaves $\boldsymbol{S}_{\text {billiard }}=\left(\Sigma_{1}, \Sigma_{2}, \Sigma_{3}, R_{1}, R_{3}, N_{1}\right)$ as the state vector.

The system of differential equations on $\mathcal{O}_{\mathcal{B}}$ is given by

$$
\begin{aligned}
\partial_{\tau} \Sigma_{1} & =2\left(1-\Sigma^{2}\right) \Sigma_{1}-2 R_{3}^{2}+\frac{2}{3} N_{1}^{2}, \\
\partial_{\tau} \Sigma_{2} & =2\left(1-\Sigma^{2}\right) \Sigma_{2}-2\left(R_{1}^{2}-R_{3}^{2}\right)-\frac{1}{3} N_{1}^{2}, \\
\partial_{\tau} \Sigma_{3} & =2\left(1-\Sigma^{2}\right) \Sigma_{3}+2 R_{1}^{2}-\frac{1}{3} N_{1}^{2}, \\
\partial_{\tau} R_{1} & =2\left(1-\Sigma^{2}\right) R_{1}+\left(\Sigma_{2}-\Sigma_{3}\right) R_{1}, \\
\partial_{\tau} R_{3} & =2\left(1-\Sigma^{2}\right) R_{3}+\left(\Sigma_{1}-\Sigma_{2}\right) R_{3}, \\
\partial_{\tau} N_{1} & =-2\left(\Sigma^{2}+\Sigma_{1}\right) N_{1},
\end{aligned}
$$


where

$$
\Sigma^{2}=\frac{1}{6}\left(\Sigma_{1}^{2}+\Sigma_{2}^{2}+\Sigma_{3}^{2}+2 R_{1}^{2}+2 R_{3}^{2}\right) .
$$

The Gauss constraint $(5.6 \mathrm{j})$ reads

$$
1-\Sigma^{2}-\frac{1}{12} N_{1}^{2}=0
$$

and the Codazzi constraint (5.6k) takes the form

$$
R_{3} N_{1}=0 .
$$

Due to the Codazzi constraint, the billiard subset consists of two invariant components: the component $N_{1}=0$ is the Bianchi type I subset $\overline{\mathcal{B}}_{R_{3} R_{1}}$, which consists of $\mathrm{K}^{\bigcirc} \cup \mathcal{B}_{R_{1}} \cup \mathcal{B}_{R_{3}} \cup \mathcal{B}_{R_{3} R_{1}}$; the component $N_{1} \neq 0$ is the Bianchi type II subset, which is given by $\mathcal{B}_{R_{1}} \cup \mathcal{B}_{N_{1}} \cup \mathcal{B}_{R_{1} N_{1}}$. The flow of the dynamical system on these subsets has been analyzed in Section 6 . A depiction of the flow on the boundary subsets $\mathcal{B}_{R_{1}}, \mathcal{B}_{R_{3}}$ (single frame transitions $\mathcal{T}_{R_{1}}, \mathcal{T}_{R_{3}}$ ) and $\mathcal{B}_{N_{1}}$ (single curvature transitions $\mathcal{T}_{N_{1}}$ ) is given in figure 3 ; for the flow on $\mathcal{B}_{R_{3} R_{1}}$ (double frame transitions $\mathcal{T}_{R_{3} R_{1}}$ ) and $\mathcal{B}_{R_{1} N_{1}}$ (mixed curvature transitions $\mathcal{T}_{R_{1} N_{1}}$ ), see figure 4 . The analysis of the stability of the fixed points on $\mathrm{K}^{\circ}$, as regards $\mathcal{O}_{\mathcal{B}}$, is summarized in figure 7 .

A sequence of transitions on the billiard subset, which we denote by $\mathcal{B}_{\mathcal{T}}$, is an infinite concatenation of transition orbits on $\mathcal{O}_{\mathcal{B}}$. Each transition $\mathcal{T}_{i}$ of $\mathcal{B}_{\mathcal{T}}$ is either a frame transition $\mathcal{T}_{R_{1}}, \mathcal{T}_{R_{3}}, \mathcal{T}_{R_{1} R_{3}}$, or a curvature transition $\mathcal{T}_{N_{1}}, \mathcal{T}_{R_{1} N_{1}}$. It is understood that the final state of each transition $\mathcal{T}_{i}$ (which is a fixed point on $\mathrm{K}^{\bigcirc}$ ) coincides with the initial state of the transition $\mathcal{T}_{i+1}$. Clearly, billiard sequences $\mathcal{B}_{\mathcal{T}}$ can be viewed as a special case of the more general sequences of transitions $\mathcal{S}_{\mathcal{T}}$ on $\mathcal{O}$.

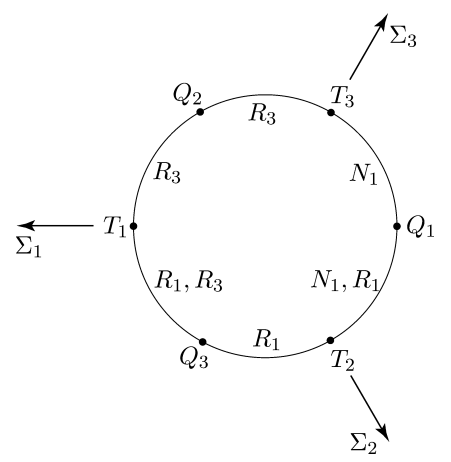

Figure 7: Active triggers on $\mathrm{K}^{\bigcirc}$ associated with the billiard subset; note that $R_{3} N_{1}=0$. 


\subsection{The asymptotic suppression of multiple transitions}

The considerations of Section 10 can be summarized as follows: the dynamical evolution of generic solutions $\boldsymbol{X}$ is asymptotically silent and local, and the asymptotic behavior along generic timelines is governed by the dynamics on the SH silent boundary. At a spatial point $x^{i}$, the asymptotic evolution of $\boldsymbol{X}(\tau)$ is thus represented by a solution $\boldsymbol{S}=\left(\Sigma_{\alpha \beta}, A_{\alpha}, N_{\alpha \beta}\right)$ of the finite dimensional SH dynamical system. The stable variables $\boldsymbol{S}_{\text {stable }}=$ $\left(N_{12}, N_{23}, N_{31}, A_{\alpha}\right)$ vanish in the limit $\tau \rightarrow 0$. Among the remaining oscillatory variables, which are $\boldsymbol{S}_{\mathrm{osc}}=\left(\Sigma_{\alpha}, R_{\alpha}, N_{1}, N_{2}\right)$, two more variables converge to zero: $R_{2}$ and $N_{2}$. This leaves the billiard variables $\boldsymbol{S}_{\text {billiard }}=$ $\left(\Sigma_{\alpha}, R_{1}, R_{3}, N_{1}\right)$ as the variables that capture the essential asymptotic dynamics.

The billiard variables are constrained by the asymptotic condition

$$
N_{1} R_{3} \rightarrow 0 \quad(\tau \rightarrow \infty)
$$

which is the asymptotic Codazzi constraint that ensues from (10.1). In the same limit the variables $\boldsymbol{S}_{\text {billiard }}$ satisfy the billiard system (11.1a) and (11.1f) and the Gauss constraint (11.1g) holds. The asymptotic evolution of $\boldsymbol{S}_{\text {billiard }}$ is thus governed by the dynamics on the billiard subset $\mathcal{O}_{\mathcal{B}}$; in other words: the state vectors $\boldsymbol{S}_{\text {billiard }}$ of solutions $\boldsymbol{X}(\tau)$ shadow sequences of transitions $\mathcal{B}_{\mathcal{T}}$ on the billiard subset. Accordingly, in the asymptotic regime, the orbit $\boldsymbol{S}_{\text {billiard }}(\tau)$ can be partitioned into a sequence of segments, where each segment is associated with a transition on $\mathcal{O}_{\mathcal{B}}$. We call this representation of the orbit $\boldsymbol{S}_{\text {billiard }}(\tau)$ an asymptotic billiard sequence $\mathcal{A B}_{\mathcal{T}}$. Since a billiard sequence $\mathcal{B}_{\mathcal{T}}$ on $\mathcal{O}_{\mathcal{B}}$ is a concatenation of transitions of the types $\mathcal{T}_{N_{1}}, \mathcal{T}_{R_{1}}, \mathcal{T}_{R_{3}}, \mathcal{T}_{R_{3} R_{1}}, \mathcal{T}_{N_{1} R_{1}}$, an asymptotic billiard sequence $\mathcal{A} \mathcal{B}_{\mathcal{T}}$ is a concatenation of approximate transitions, i.e., $\mathcal{A B}_{\mathcal{T}}=\left(\mathcal{T}_{i}\right)_{i \in \mathbb{N}}$, where each $\mathcal{T}_{i}$ denotes an approximate transition in the present context.

In asymptotic billiard sequences $\mathcal{A B}_{\mathcal{T}}$, however, multiple transitions are suppressed asymptotically, i.e., transitions of the types $\mathcal{T}_{R_{3} R_{1}}, \mathcal{T}_{N_{1} R_{1}}$ do not occur in the asymptotic regime. In the following we discuss this statement; however, we focus on the simpler case of $\mathcal{T}_{R_{3} R_{1}}$ transitions.

From the differential equations (11.1d) and (11.1e), we obtain

$$
\partial_{\tau} \log \frac{R_{1}}{R_{3}}=3 \Sigma_{2} \quad \text { and thus } \quad \log \frac{R_{1}}{R_{3}} \propto 3 \int \Sigma_{2} d \tau,
$$

where we assume for simplicity and without loss of generality that $R_{1}$, $R_{3}>0$. In an asymptotic billiard sequence $\mathcal{A} \mathcal{B}_{\mathcal{T}}$, the variables $R_{1}$ and $R_{3}$ (as 
well as $N_{1}$ ) are characterized by oscillations between zero and finite maxima (which are bounded above via the Gauss constraint); in particular, $R_{1} \nrightarrow \rightarrow 0$ and $R_{3} \nrightarrow \rightarrow 0$ as $\tau \rightarrow \infty$ (as well as $N_{1} \nrightarrow \nrightarrow 0$ ). For $\mathcal{T}_{R_{1}}$ transitions, while $R_{1}$ is of order unity, $R_{3}$ is small, so that $R_{1} / R_{3}$ is large; conversely, for $\mathcal{T}_{R_{3}}$ transitions, $R_{1} / R_{3}$ is small.

Consequently, $\log \left(R_{1} / R_{3}\right)$ and thus $\int \Sigma_{2} d \tau$ is a function that oscillates in the range $(-\infty, \infty)$. The central property of these oscillations is that the amplitude increases (rapidly) with increasing $\tau$, which is due to the increasing accuracy of the shadowing. In the asymptotic regime we can use a simple model for these oscillations, which is based on the observation that the orbit $\mathcal{A B}_{\mathcal{T}}$ spends an increasing amount of $\tau$-time in a neighborhood of the Kasner fixed points, while each transition always takes a fixed $\Delta \tau$ (depending on the transition). We obtain $\int \Sigma_{2} d \tau=\sum\left(\Sigma_{2}\right)_{i}(\Delta \tau)_{i}$, where $i \in \mathbb{N}$ consecutively numbers the Kasner fixed points that the orbit passes, and where, accordingly, $(\Delta \tau)_{i}$ is the time the orbit spends in a neighborhood of the Kasner point $i$. The quantities $\left(\Sigma_{2}\right)_{i}$ range in $(-2,2)$; in conformance with our assumptions, we expect the distribution of the $\left(\Sigma_{2}\right)_{i}$ in $(-2,2)$ to have a probabilistic description. Therefore, the sum $\sum\left(\Sigma_{2}\right)_{i}(\Delta \tau)_{i}$ can be thought of as arising from a stochastic process resembling a random walk $\sum s_{i}$; in the present case, however, the step size $s_{i}$ is not fixed, but itself random; most importantly, the step size increases with the number of steps taken.

The growth of the step size is of crucial importance. Consider an interval $(-a, a)$ (where $a$ is small compared to the first step size). If $s_{i}$ increases slowly with $i$, the probability that $\sum_{i=1}^{n} s_{i} \in(-a, a)$ for some $n$ (recurrence probability) is one. If the growth is sufficiently fast, however, this probability is zero; this is case, for instance, if the growth rate is geometric. The behavior of the quantity $\log \left(R_{1} / R_{3}\right)$ is determined accordingly: for every $a$, there exists $\tau_{a}$ such that the average step size is large compared to $a$ for all $\tau>\tau_{a}$; the probability that $\log \left(R_{1} / R_{3}\right)$ lies in the interval $(-a, a)$ for some $\tau>\tau_{a}$ is expected to vanish; this is because the step size increases rapidly, which is in turn due to the expectation that the orbit $\mathcal{A B}_{\mathcal{T}}$ shadows the attractor with a rapidly increasing accuracy (so that $(\Delta \tau)_{i}$ grows fast). Consequently, in the asymptotic regime, the probability of the quantity $\log \left(R_{1} / R_{3}\right)$ being in any given interval around zero vanishes. Since, therefore, either $R_{1}$ is small and $R_{3}$ large or $R_{1}$ large or $R_{3}$ small, our arguments suggest that multiple transitions are suppressed as $\tau \rightarrow \infty$.

\subsection{The billiard attractor}

The asymptotic suppression of multiple transitions in the asymptotic regime entails that asymptotic billiard sequences $\mathcal{A B}_{\mathcal{T}}$ do not approach general 
billiard sequences $\mathcal{B}_{\mathcal{T}}$ on the billiard subset $\mathcal{O}_{\mathcal{B}}$ but sequences on the billiard attractor $\mathcal{O}_{\mathcal{B A}}$.

We define the billiard attractor subset $\mathcal{O}_{\mathcal{B} \mathcal{A}}$ as

$$
\mathcal{O}_{\mathcal{B} \mathcal{A}}=\mathrm{K}^{\bigcirc} \cup \mathcal{B}_{N_{1}} \cup \mathcal{B}_{R_{1}} \cup \mathcal{B}_{R_{3}},
$$

i.e., $\mathcal{O}_{\mathcal{B A}}$ is the boundary of the billiard subset $\mathcal{O}_{\mathcal{B}}$.

An attractor sequence of transitions $\mathcal{A}_{\mathcal{T}}$ (or, for brevity, attractor sequence) is a sequence of transitions on the billiard attractor $\mathcal{O}_{\mathcal{B} \mathcal{A}}$. By definition, $\mathcal{A}_{\mathcal{T}}$ is an infinite concatenation of single transitions of the type $\mathcal{T}_{N_{1}}, \mathcal{T}_{R_{1}}$, and $\mathcal{T}_{R_{3}}$, i.e., one type of single curvature transition and two types of single frame transitions; to get an intuitive picture of attractor sequences, we simply refer to figure $3(\mathrm{a})$ to (c).

The collection of our results leads to the formulation of the dynamical systems billiard conjecture:

Conjecture. The asymptotic dynamical evolution of a generic timeline of a solution of Einstein's vacuum equations (expressed in an Iwasawa frame) that exhibits a generic spacelike singularity is characterized as follows:

(i) It is asymptotically silent and local.

(ii) In the asymptotic limit, the essential dynamics is represented by an attractor sequence $\mathcal{A}_{\mathcal{T}}$ on the billiard attractor $\mathcal{O}_{\mathcal{B} \mathcal{A}}$.

The dynamical systems billiard conjecture can be viewed as the "dual formulation" of the cosmological billiard conjecture by Damour et al. [13]. In Section 12 we will establish in detail the correspondence between the two approaches. In the remainder of this section, we will give a brief summary of our analysis that has led to the dynamical systems billiard conjecture.

Our previous analysis constitutes a derivation of the dynamical systems billiard conjecture; however, although a considerable part of our treatment meets the criterion of mathematical rigor, some of our arguments are heuristic rather than rigorous (despite their being mathematically convincing); therefore, the presented derivation of the billiard conjecture does not represent a rigorous proof but merely a first step toward a rigorous treatment. Nonetheless, apart from the fact that our analysis provides strong support for the billiard conjecture, we expect that many aspects of our considerations are fundamental for a deeper understanding of the asymptotic dynamics associated with generic spacelike singularities; in particular we believe that we have identified several issues that have to be taken into account in the pursuit of rigor. 
Let us therefore recapitulate the main steps in our derivation. Hereby we are guided by the uncovered hierarchical structure of (boundary) subsets: the essential asymptotic dynamics can be restricted successively to subsets of subsets, to boundaries of boundaries.

A Assumption: Asymptotic silence and asymptotically local dynamics. Asymptotically local dynamics is defined by the requirement that $E_{\alpha}{ }^{i}$ $\rightarrow 0, \boldsymbol{\partial}_{\alpha}\left(\boldsymbol{S}, r_{\beta}, \dot{U}_{\beta}\right) \rightarrow 0,\left(r_{\alpha}, \dot{U}_{\alpha}\right) \rightarrow 0$, which leads to a spatial decoupling of the field equations. As a consequence, a generic solution $\boldsymbol{X}(\tau)$ of the Einstein vacuum equations at a generic spatial point approaches the SH part of the silent boundary $E_{\alpha}{ }^{i}=0$, which is spanned by the state vector $\boldsymbol{S}=\left(\Sigma_{\alpha \beta}, A_{\alpha}, N_{\alpha \beta}\right)$.

$\Rightarrow$ B Assumption: Asymptotic dominance of the Kasner states in the asymptotic evolution of solutions. Linear analysis of the Kasner circle $\mathrm{K}^{\bigcirc}$ leads to the conjecture that the variables $\boldsymbol{S}_{\text {stable vanish in the asymp- }}$ totic limit $\tau \rightarrow \infty$, where $\boldsymbol{S}_{\text {stable }}=\left(N_{\alpha \beta}, A_{\alpha}\right)(\alpha \neq \beta)$. Hence the essential asymptotic dynamics is represented by the dynamics on a subset of the SH part of the silent boundary, the oscillatory subset $\mathcal{O}$, and its associated state vector $\boldsymbol{S}_{\text {osc }}=\left(\Sigma_{\alpha}, R_{\alpha}, N_{1}, N_{2}\right)$. Orbits on $\mathcal{O}$ are heteroclinic orbits (in general, transitions) that can be joined to form infinite sequences. These sequences of orbits/transitions are accessible to a mathematically rigorous treatment, where stochastic aspects become important.

$\Rightarrow$ C Assumption: Asymptotic shadowing. A generic solution $\boldsymbol{X}(\tau)$ shadows a sequence of orbits/transitions on $\mathcal{O}$ with an increasing degree of accuracy. Our analysis shows that we have $R_{2} \rightarrow 0$ and $N_{2} \rightarrow 0$ along generic orbits $\boldsymbol{X}(\tau)$; this implies that certain types of transitions that involve the variables $R_{2}, N_{2}$ are ruled out a priori (complete exclusion), while it can shown that the probability for the occurrence of any of the other transitions involving these variables is zero (stochastic exclusion). The asymptotic exclusion of $R_{2}$ and $N_{2}$ entails that the essential asymptotic dynamics of $\boldsymbol{X}$ is described by the dynamics on the billiard subset $\mathcal{O}_{\mathcal{B}}$ with its state vector $\boldsymbol{S}_{\text {billiard }}=\left(\Sigma_{1}, \Sigma_{2}, \Sigma_{3}, R_{1}, R_{3}, N_{1}\right)$.

$\Rightarrow$ D Asymptotic shadowing of billiard sequences. A generic solution $\boldsymbol{X}(\tau)$ shadows a sequence of transitions on $\mathcal{O}_{\mathcal{B}}$ (billiard sequence) with an increasing degree of accuracy. Our arguments indicate that the variables $\left(R_{1}, R_{3}, N_{1}\right)$ of $\boldsymbol{S}_{\text {billiard }}$ cannot be excited simultaneously. This leads to the conclusion that the generic asymptotic dynamics is governed by the flow on the boundary of the billiard subset, which is the billiard attractor $\mathcal{O}_{\mathcal{B A}}=\mathrm{K}^{\bigcirc} \cup \mathcal{B}_{N_{1}} \cup \mathcal{B}_{R_{1}} \cup \mathcal{B}_{R_{3}}$. The dynamics on this attractor set is represented by billiard attractor sequences (consisting of one type of curvature transitions and two types of frame transitions: $\mathcal{T}_{N_{1}}$, and $\mathcal{T}_{R_{1}}, \mathcal{T}_{R_{3}}$ ), which thus form the attractor for generic solutions 
$\boldsymbol{X}$. In other words, the essential dynamics of a generic solution $\boldsymbol{X}$ is characterized by its approach to an attractor sequence $\mathcal{A}_{\mathcal{T}}$.

In this paper, we have given heuristic arguments for $\mathrm{A} \Rightarrow \mathrm{B}$, but our main focus has been the other steps. Assuming that generic solutions $\boldsymbol{X}$ are asymptotic sequences of $\mathcal{O}$-orbits (i.e., assuming shadowing of the flow on $\mathcal{O}$ ) has inevitably led to the implication $\mathrm{B} \Rightarrow \mathrm{D}$.

Since stochastic considerations are essential for parts of our considerations, we obtain only statements for (generic timelines of) generic solutions. The possibility exists that there are solutions violating one or the other properties; however, such solutions are expected to form a set of measure zero in the space of all solutions.

\section{2 "Duality" of Hamiltonian and dynamical systems billiards}

In Section 2 we have outlined the Hamiltonian approach to cosmological billiards, where we have followed Damour, Hennaux, and Nicolai $[13,14]$. This approach is based on an analysis of the Hamiltonian $\mathcal{H}$ in Iwasawa frame variables, which are the diagonal degrees of freedom $b^{\alpha}$, the off-diagonal variables $\mathcal{N}^{\alpha}{ }_{i}$, and the conjugate momenta. An asymptotic Hamiltonian $\mathcal{H}_{\infty}$ is constructed from $\mathcal{H}$ by taking the limit $\rho=\sqrt{-b_{\alpha} b^{\alpha}} \rightarrow \infty$ and dropping all terms except for three terms that are identified as being "dominant". The asymptotic Hamiltonian $\mathcal{H}_{\infty}$ is assumed to describe the generic asymptotic dynamics of generic solutions that exhibit a spacelike singularity.

A direct consequence from the form of the asymptotic Hamiltonian $\mathcal{H}_{\infty}$ is that the off-diagonal degrees of freedom are asymptotic constants of the motion, as is a momentum variable associated with a projection of the diagonal degrees of freedom - a phenomenon referred to as "asymptotic freezing"; for a derivation of these results using the present dynamical systems formalism and the results from the previous sections, see Appendix H. Therefore, the non-trivial asymptotic dynamics is encoded in the diagonal degrees of freedom, or, more precisely, their projections $\gamma^{\alpha}$ : the asymptotic dynamics at each spatial point is described as a geodesic motion in a portion of hyperbolic space that is bounded by sharp walls, see Section 2 and $[13,14]$. This picture prompts the terminology "billiard motion" and "cosmological billiards"; see Figure 8(a).

It is of interest to consider a Hamiltonian that is in a sense a link between $\mathcal{H}$ and $\mathcal{H}_{\infty}$ : the "dominant" Hamiltonian $\mathcal{H}_{\text {dom }}$. It is obtained by dropping 


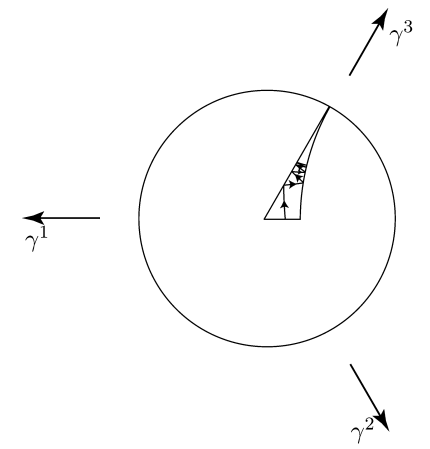

(a) The cosmological billiard

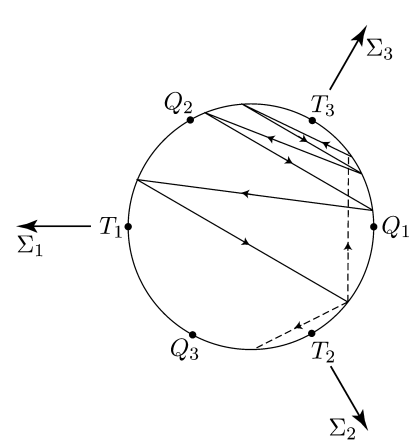

(b) The billiard attractor

Figure 8: Part of an orbit in terms of free Kasner (Fermi frame) motion and frame and curvature bounces. This represents a 'configuration space' projection of the asymptotic dynamics. The disc here represents hyperbolic space. Figure (b) shows part of an orbit in terms of single frame and curvature transitions, i.e., it shows a part of an attractor sequence. Note that the solution does not quite return to any of the Kasner points it has 'visited' before. This description represents a 'momentum space' projection of the asymptotic dynamics. The circle here is the Kasner circle $\mathrm{K}^{\bigcirc}$. The dashed lines correspond to the two possible single transitions that are possible at this stage; which one is realized depends on initial data. This corresponds to that free motion in a given direction in (a) may either lead to that one hits the wall associated with $R_{1}$ (the short wall) or $N_{1}$ (the curved wall).

the "subdominant" terms of $\mathcal{H}$ before the limit $\rho \rightarrow \infty$ is taken. Since the asymptotic Hamiltonian constructed from $\mathcal{H}_{\text {dom }}$ naturally coincides with $\mathcal{H}_{\infty}$, the asymptotic dynamics described by $\mathcal{H}$ and $\mathcal{H}_{\text {dom }}$ is the same.

From Section 2 and $[13,14]$, we obtain the dominant Hamiltonian:

$$
\mathcal{H}_{\mathrm{dom}}=\tilde{N}\left[\frac{1}{4} \mathcal{G}^{\alpha \beta} \pi_{\alpha} \pi_{\beta}+\frac{1}{2} \tilde{R}_{1}^{2} e^{2\left(b^{2}-b^{3}\right)}+\frac{1}{2} \tilde{R}_{3}^{2} e^{2\left(b^{1}-b^{2}\right)}+\frac{1}{8} \tilde{N}_{1}^{2} e^{-4 b^{1}}\right],
$$

where $\tilde{R}_{1}:=-\mathcal{P}_{3}, \tilde{R}_{3}:=-\left(\mathcal{P}_{1}+n_{3} \mathcal{P}_{2}\right)$, and where $\tilde{N}_{1}$ is a function of the variables $n_{\alpha}$ given by equation (A.16a). The non-trivial dynamics is represented by the diagonal degrees of freedom $b^{\alpha}$ (or alternatively by $\rho$ and the projected variables $\gamma^{\alpha}$ ): the dynamics is described as a motion in a portion of Lorentzian space that is bounded by "exponential walls". As the solution approaches the singularity, these walls become increasingly sharp with an infinitely high potential as limit; we refer to the remarks at the end of this section. The asymptotic dynamics is thus a free null geodesic motion in Lorentzian space interrupted by bounces at the walls, which yields an alternative representation of cosmological billiards. 
The asymptotic dynamics as described by Hamiltonian cosmological billiards can be interpreted in terms of generalized Kasner solutions: Fermipropagated generalized Kasner solutions appear as straight lines (geodesics) in flat Lorentzian space in the $b^{\alpha}$ description, or, equivalently, as geodesics in hyperbolic space in the projected $\gamma^{\alpha}$ description. A free motion between two bounces at walls thus translates to a (Fermi) "Kasner epoch", a phase where the solution evolves as a generalized Kasner solution, see (B.1). The walls and the associated bounces, on the other hand, are of two kinds: (i) frame/centrifugal/symmetry walls and associated bounces; (ii) curvature walls and associated bounces. Bounces of the former type merely result in axes permutations of a Kasner solution; bounces of the latter type are Bianchi type II bounces: the Kasner state changes in accordance with a change generated by a Bianchi type II solution.

The Hamiltonian approach emphasizes the dynamics of the configuration space variables ( $b^{\alpha}$ and the projected variables $\gamma^{\alpha}$, respectively); therefore one may say that the Hamiltonian picture yields a "configuration space" representation of the asymptotic dynamics. In the following, we will see that the dynamical systems approach can be viewed as a dual representation, i.e., a "momentum space" representation.

Consider the dominant Hamiltonian from the perspective of the dynamical systems approach. The terms $\tilde{R}_{1} \exp \left(b^{2}-b^{3}\right), \tilde{R}_{3} \exp \left(b^{1}-b^{2}\right)$, and $\tilde{N}_{1} \exp \left(-2 b^{1}\right)$ can be associated with $R_{1}, R_{3}$, and $N_{1}$ by using the relations (A.14b) and (A.14c). Accordingly, $\mathcal{H}_{\text {dom }}$ contains $\frac{1}{2}\left(R_{1}^{2}+R_{3}^{2}\right)+\frac{1}{8} N_{1}^{2}$ modulo a common factor. Recall in this context that the Hamiltonian is proportional to the Gauss constraint, cf. (11.1g). This indicates that there is an intimate connection between: (i) centrifugal bounces in the Hamiltonian picture and single frame transitions $\mathcal{T}_{R_{1}}$ and $\mathcal{T}_{R_{3}}$ in the dynamical systems picture; (ii) curvature bounces and single curvature transitions $\mathcal{T}_{N_{1}}$.

When we calculate the Hamiltonian equations of $\mathcal{H}_{\text {Dom }}=0$ associated with the non-trivial degrees of freedom $b^{\alpha}$, adopt the appropriate time gauge, and then perform suitable variable transformations according to the formulas of Appendix A, we obtain:

(i) the billiard system, i.e., the differential equations (11.1a) to (11.1f) and the Gauss constraint (11.1g), however, without the Codazzi constraint $R_{3} N_{1}=0$;

(ii) evolution equations for $E_{\alpha}=\left(E_{1}{ }^{1}, E_{2}{ }^{2}, E_{3}{ }^{3}\right)$ and the Hubble variable $H$, with all spatial frame derivatives and all variables $\boldsymbol{X}$ (and $\dot{U}_{\alpha}, r_{\alpha}$ ) set to zero except $E_{\alpha}, H$, and $\boldsymbol{S}_{\text {billiard }}$. 
The unconstrained billiard system (i) forms an independent coupled system of differential equations. The equations (ii) for $H$ and $E_{\alpha}$, on the other hand, decouple from the unconstrained billiard system. These equations can be regarded as linear equations with time dependent coefficients, which are provided by the solutions of the billiard system. (Compare with the treatment of the auxiliary quantities in Section 9). The evolution equations for $E_{\alpha}$ constitute the lowest order perturbation into the physical state space of the full equations for $E_{\alpha}$ in the vicinity of the silent boundary; together with the Hubble variable $H$, they yield $b^{\alpha}$ according to equation (A.2) in Appen$\operatorname{dix} \mathrm{A}$. The off-diagonal degrees of freedom can be treated similarly: the solutions of the unconstrained billiard system provide the time-dependent coefficients for the lowest order perturbation equations. In this manner, we obtain asymptotic freezing, see Appendix H. This result further strengthens the connection between the Hamiltonian and the dynamical systems approach.

The asymptotic limit of the dynamics of the unconstrained billiard system is represented by attractor sequences $\mathcal{A}_{\mathcal{T}}$, see figure $8(\mathrm{~b}) .{ }^{13}$ The duality of the Hamiltonian and the dynamical systems approach thus becomes apparent: one phase of free motion in the Hamiltonian billiard picture corresponds to a Kasner fixed point on the Kasner circle $\mathrm{K}^{\bigcirc}$. The underlying reason for this is that the dynamics has been projected out of the self-similar Fermipropagated Kasner solutions by means of a conformal normalization that has yielded scale-invariant variables. The bounces at walls in the Hamiltonian billiards correspond to motion in the dynamical systems picture represented by the transition orbits (heteroclinic orbits) between Kasner points. The curvature transitions $\mathcal{T}_{N_{1}}$ are non-scale-invariant solutions and correspond to curvature bounces in the Hamiltonian billiards; the frame transitions $\mathcal{T}_{R_{1}}, \mathcal{T}_{R_{3}}$ rotate axes w.r.t. a Fermi frame; see Appendix C. The transitions appear as straight lines (in Euclidian space) when projected onto $\Sigma_{\alpha}$-space. Note that the transitions and the Hamiltonian wall bounces yield exactly the same rules for changing Fermi-propagated Kasner states. (However, the Hamiltonian billiard only gives a consistent picture when we consider generic attractor sequences, which are characterized by irrational values of the Kasner parameter $u$. Rational values of the Kasner parameter $u$ lead to motion straight into the sharp corners of the asymptotic billiard where the dynamics is undefined.)

Changing from the Hamiltonian billiards to dynamical systems billiards, the notions "bounces at walls" and "motion along straight lines" switch places. In the Hamiltonian picture, free motion is followed by a bounce at a

\footnotetext{
${ }^{13} \mathrm{~A}$ more detailed discussion of this statement will be given elsewhere.
} 
wall, which again gives rise to a phase of free motion. In the dynamical systems picture, we observe "bounces" at the fixed points on the Kasner circle $\mathrm{K}^{\bigcirc}$; in fact, since the Kasner circle constitutes the boundary of $\Sigma_{\alpha}$-space, it can be viewed as a "wall" in the dynamical systems picture. Between the bounces, we have motion along straight lines in $\Sigma_{\alpha}$-space (transitions). In brief, the Hamiltonian "motion-bounce-motion-bounce" is translated to "bounce-motion-bounce-motion" in the dynamical systems billiards.

Since the variables $\Sigma_{\alpha}$ are intimately connected with the variables $\pi_{\alpha}$, it is natural to refer to the projected dynamical systems picture as a "momentum space" representation of the asymptotic dynamics, which complements the Hamiltonian configuration space representation. To compare the two pictures, see figure 8 .

The correspondence between the dynamical systems and Hamiltonian pictures offers a possibility for mutual support. Let us give a few examples: (i) As can be inferred from figures 5 and 8 , the typical "captured" $\mathcal{T}_{N_{1}}-\mathcal{T}_{R_{3}}$ oscillatory behavior (a small curvature phase) corresponds to the many oscillations in the upper right corner in the Hamiltonian billiard, see figure 8(a). (ii) Figure 7 indicates that $\mathcal{T}_{R_{1}}$ transitions are less frequent than $\mathcal{T}_{R_{3}}$ transitions: $R_{1}$ and $R_{3}$ are unstable in three sectors of $\mathrm{K}^{\bigcirc}$; however, $R_{1}$ has to compete with $N_{1}$ in sector (132), which "depletes" $\mathcal{T}_{R_{1}}$ transition at the cost of $\mathcal{T}_{N_{1}}$ transitions; $\mathcal{T}_{R_{3}}$ is unaffected by this. The correctness of these arguments is corroborated by the features of Lobashevski geometry close to the circular boundary and by the fact that the wall associated with $\mathcal{T}_{R_{3}}$ bounces is longer than the one associated with $\mathcal{T}_{R_{1}}$ bounces. (iii) Consider the Hamiltonian $\mathcal{H}_{\text {dom }}$ and perform a projection of the dynamics onto spatial constant volume slices in $b^{\alpha}$-space. The walls are "exponential walls" whose contours are of a triangular shape where the corners are smoothed out. Toward the singularity (i.e., with shrinking volume) the triangle increases in size, where simultaneously the triangle's sides become increasingly straight and the corners increasingly sharp (relative to the size of the triangle), see [11] for similar SH examples. Particularly, the corner shape is sensitive to the additive effects from the different walls potentials. The increasing size of the potential contour triangle and the comparatively sharper corners suggest that it becomes increasingly less likely that the "billiard ball" hits the corners. This geometrical feature suggests that, statistically, multiple transitions are asymptotically suppressed, in agreement with our quantitative analysis.

It is of interest to also compare the dynamical systems picture with previous (Hamiltonian) treatments of so-called asymptotic velocity term dominated (AVTD) dynamics; this is done in Appendix I. 


\section{Models with symmetries in an Iwasawa frame}

In this section, we give a brief overview on models with symmetries. Such models are of obvious significance for the study of cosmological singularities; since the equations simplify, we can hope to obtain more substantial evidence and perhaps even prove theorems on the asymptotic dynamics of solutions. This in turn can be expected to shed new light on the heuristic statements concerning the generic case without symmetries.

\section{1 $G_{1}$ models}

By definition, cosmological $G_{1}$ models possess one spacelike Killing vector; let us choose this Killing vector to be $\partial_{x^{1}}$. The Iwasawa decomposition is compatible with $G_{1}$ symmetry and we are therefore able to choose an Iwasawa frame that is symmetry adapted; we set $\partial_{\alpha}=E_{\alpha}{ }^{i} \partial_{x^{i}}$, where $E_{\alpha}{ }^{i}$ is a lower triangular matrix, see Section 3; accordingly,

$$
\boldsymbol{\partial}_{1}=E_{1}^{1} \partial_{x^{1}}, \quad \boldsymbol{\partial}_{2}=E_{2}^{1} \partial_{x^{1}}+E_{2}^{2} \partial_{x^{2}}, \quad \boldsymbol{\partial}_{3}=E_{3}{ }^{1} \partial_{x^{1}}+E_{3}^{2} \partial_{x^{2}}+E_{3}^{3} \partial_{x^{3}},
$$

where $E_{\alpha}{ }^{i}$ as well as $H$ and $\mathcal{N}$ are independent of $x^{1}$. Consequently, when $f$ is a function that is independent of $x^{1}$, it follows that

$$
\partial_{1} f=0, \quad \partial_{2} f=E_{2}^{2} \partial_{x^{2}} f, \quad \partial_{3} f=\left(E_{3}^{2} \partial_{x^{2}}+E_{3}^{3} \partial_{x^{3}}\right) f
$$

This in turn leads to a decoupling of the $E_{\alpha}{ }^{1}$ equations from the system (3.6) to (3.10), since $E_{\alpha}{ }^{1} \partial_{x^{1}}$ disappears from the r.h.s. of the evolution equations and the constraints. Note also that (3.7a) and (3.8a) do not mix the components $E_{\alpha}{ }^{1}$ with $E_{\alpha}{ }^{2}$ and $E_{\alpha}{ }^{3}$. In addition, using the expressions in Appen$\operatorname{dix} \mathrm{A}$, the symmetries are seen to further reduce the state space (3.12) and (3.14); since

$$
N_{22}=N_{23}=A_{1}=\dot{U}_{1}=r_{1}=0,
$$

the state space associated with the reduced coupled system of equations is given by

$$
\boldsymbol{X}=\left(E_{2}^{2}, E_{3}^{2}, E_{3}^{3}, \Sigma_{\alpha}, R_{\alpha}, N_{1 \alpha}, A_{2}, A_{3}\right) ;
$$

in addition $\dot{U}_{2}, \dot{U}_{3}, r_{2}, r_{3}$ are non-zero for most temporal gauge choices.

Apart from the above generic $G_{1}$ case, there exist special $G_{1}$ models. The hypersurface-orthogonal Killing vector case, also referred to as the polarized case in a $U(1)$ context, see [38] and references therein, is given by setting $E_{2}{ }^{1}$ and $E_{3}{ }^{1}$ to zero, so that the metric takes a diagonal form. Using the expressions in Appendix A, it follows that $N_{11}=R_{2}=R_{3}=0$. In this case, 
the sectors (231), (213), and (123) on the Kasner circle $\mathrm{K}^{\bigcirc}$ become stable, which in turn implies that solutions asymptotically approach a Kasner state; for proofs, see $[38,39]$.

\section{2 $G_{2}$ models}

Models with two commuting spacelike Killing vectors are known as $G_{2}$ models, see, e.g., [12]. An Iwasawa frame that is adapted to the symmetries is obtained by letting $\boldsymbol{\partial}_{1}$ and $\boldsymbol{\partial}_{2}$ be tangent to the group orbits. Accordingly, $E_{\alpha}{ }^{i}$ and $H, \mathcal{N}$ are functions that are independent of $x^{1}$ and $x^{2}$ and thus depend on $x^{0}$ and $x^{3}$ only. For any $f=f\left(x^{0}, x^{3}\right)$, we get

$$
\partial_{1} f=0, \quad \partial_{2} f=0, \quad \partial_{3} f=E_{3}{ }^{3} \partial_{x^{3}} f ;
$$

furthermore, as follows from Appendix A,

$$
N_{22}=N_{13}=N_{23}=N_{33}=A_{1}=A_{2}=\dot{U}_{1}=\dot{U}_{2}=r_{1}=r_{2}=0 .
$$

In addition, one can exploit the remaining freedom in choosing the frame at a spatial point to obtain $R_{2}=0$, and then the Codazzi constraint and the evolution equation for $R_{2}$ force $R_{2}$ to be identically zero everywhere [40]. Using a symmetry-adapted Iwasawa frame thus leads to a decoupling of all $E_{\alpha}{ }^{i}$ equations, except for the equation for $E_{3}{ }^{3}$. Accordingly, the reduced state space for the problem is represented by the state vector

$$
\boldsymbol{X}=\left(E_{3}^{3}, \Sigma_{\alpha}, R_{1}, R_{3}, N_{11}, N_{12}, A_{3}\right) ;
$$

in addition $\dot{U}_{3}, r_{3}$ are non-zero for most temporal gauge choices. If one in addition chooses the timelike separable area gauge [41], so that the area density of the $G_{2}$ symmetry orbits, i.e., $\mathcal{A}=\left(e_{1}{ }^{1} e_{2}{ }^{2}\right)^{-1}$, is a function of $x^{0}$ only, then $A_{3}=r_{3}$, as follows from (3.8a).

There exist several special classes of $G_{2}$ models:

(i) Hypersurface-orthogonal models are characterized by the restriction $N_{11}=R_{3}=0$.

(ii) Orthogonally transitive models are obtained by setting $R_{1}=0$.

(iii) The diagonal case is given by $N_{11}=R_{1}=R_{3}=0$; this case is also referred to as the polarized case in the literature dealing with a $T^{3}$ topology $[42,43]$.

(iv) Plane symmetric models are determined by $N_{11}=N_{12}=R_{1}=R_{3}=0$, $\Sigma_{11}=\Sigma_{22}$. 
In [22] slightly different variables were used to discuss the general case with $T^{3}$-topology; these can be obtained as follows: firstly, note that $\dot{U}_{\alpha}=$ $\dot{u}_{\alpha} / H-r_{\alpha}, A_{\alpha}=a_{\alpha} / H+r_{\alpha}$, where $\dot{u}_{\alpha}, a_{\alpha}$ are the acceleration and spatial connection coefficients associated with $\mathbf{g}$, respectively. Secondly, to identify the present frame with the one used in [22], we permute both frame and coordinate indices from the present ones to the ones used in [22] according to $1 \rightarrow 3,2 \rightarrow 1,3 \rightarrow 2$. Then, in addition, we make the few scalings and notational changes that are needed in order to obtain the variables $\left(E_{1}{ }^{1}, r, \dot{U}, \Sigma_{+}, \Sigma_{-}, \Sigma_{\times}, \Sigma_{2}, N_{-}, N_{\times}\right)$used in [22] in terms of the present ones; $E_{3}{ }^{3}$ is replaced with $E_{1}{ }^{1}$, which was used in [22], while the other variables are related as follows:

$$
\begin{aligned}
r & =r_{3}=A_{3}, & \dot{U} & =r_{3}+\dot{U}_{3}, & & \\
\Sigma_{+} & =\frac{1}{2}\left(\Sigma_{11}+\Sigma_{22}\right), & \Sigma_{-} & =\frac{1}{2 \sqrt{3}}\left(\Sigma_{11}-\Sigma_{22}\right), & & \Sigma_{\times}=-\frac{1}{\sqrt{3}} R_{3}, \\
\Sigma_{2} & =-\frac{1}{\sqrt{3}} R_{1}, & N_{-} & =\frac{1}{2 \sqrt{3}} N_{11}, & & N_{\times}=\frac{1}{\sqrt{3}} N_{12} .
\end{aligned}
$$

For our present purposes, it is of interest to note that numerical experiments performed in the general $G_{2}$ case in [22] gave evidence for the claim that multiple transitions become increasingly rare and that the billiard attractor gives a correct generic asymptotic description. However, the results in the present paper suggest that it would be useful to numerically check our more detailed statements about asymptotic suppression and decay rates (as well as freezing, as discussed in Appendix $\mathrm{H}$ ); the same also holds for the next class of models.

\subsection{Generic Bianchi type $\mathrm{VI}_{-1 / 9}$ models}

The evolution and constraint equations for $\mathrm{SH}$ cosmologies are given by setting all terms involving spatial derivatives to zero, which includes setting $\dot{U}_{\alpha}=0=r_{\alpha}$, see also Sections 3 and 4. This implies that the conformal Hubble normalization becomes equivalent with the usual Hubble normalization used in, e.g., [12]. While the Iwasawa gauge is compatible with the symmetry-adapted frames of Bianchi types I-VII, since they admit a subgroup of two spacelike commuting Killing vectors, it is incompatible with the symmetry-adapted frames of Bianchi types VIII and IX. The most general model with a symmetry-adapted Iwasawa frame is the general Bianchi type $\mathrm{VI}_{-1 / 9}$ model, also sometimes referred to as the exceptional type $\mathrm{VI}_{h}$ model.

The asymptotic dynamics of generic Bianchi cosmologies of type $\mathrm{VI}_{-1 / 9}$ has been studied in [36]. The compatibility of these models with the Iwasawa 
gauge is responsible for their particular significance in the context of generic inhomogeneous cosmologies: the general type $\mathrm{VI}_{-1 / 9}$ case is the only $\mathrm{SH}$ vacuum case where models possess the same attractor toward the singularity as generic inhomogeneous cosmologies in an Iwasawa frame; in particular, they exhibit oscillatory asymptotic behavior. The type $\mathrm{VI}_{-1 / 9}$ models admit a $G_{2}$ subgroup; adapting the spatial Iwasawa frame to the three-dimensional symmetry surfaces so that the first two vectors are adapted to the $G_{2}$ subgroup implies that the same quantities as in the $G_{2}$ case become zero; however, the extra symmetry forces some additional quantities to be zero as well. The Codazzi constraint with $\alpha=2$ yields $R_{1}\left(N_{12}-3 A_{3}\right)=0$, but, in contrast to other type $\mathrm{VI}_{h}$ models, $h=-1 / 9$ implies, with the present conventions, that $N_{12}=3 A_{3}$; hence $R_{1}$ is not forced to vanish since the Codazzi constraint is automatically satisfied, see, e.g., [12]; the general type $\mathrm{VI}_{-1 / 9}$ case is thus characterized by non-zero off-diagonal shear components $R_{1}$ and $R_{3}$. It follows that we can choose

$$
\boldsymbol{S}=\left(\Sigma_{1}, \Sigma_{2}, R_{1}, R_{3}, N_{1}, A_{3}\right),
$$

as the reduced state space variables.

Using the variables (13.1) with the opposite signs ${ }^{14}$ for $\Sigma_{2}$ and $\Sigma_{\times}$, and $r_{\alpha}=\dot{U}_{\alpha}=0$, yields the system given in [36], where a numerical investigation indicated that multiple transitions become increasingly rare and that the billiard attractor gives a correct generic asymptotic description, in agreement with the present general analysis.

\section{Gauge considerations}

It is a matter of course that there exist many useful spatial frame choices; while our present considerations are based on the choice of an Iwasawa frame, other prominent gauges are: the frame used in UEWE [20]; the $\mathrm{SO}(3)$ choice used by Benini and Montani as the starting point for a Misner/Chitré billiard analysis [44]; the Fermi choice $\left(R_{\alpha}=0\right)$. Each of these gauges exhibits some computational advantages over the others in special contexts, and each has its physical merits: e.g., Fermi observers associated with Fermi frames do not get dizzy. But it is only in special situations, when one considers an important class of spacetimes that shares a special, often global, feature, that one can argue that a particular frame choice is preferred. It is probably safe to conclude that no single frame choice will be optimal as regards all features one may be interested in; e.g., Iwasawa frames are incompatible

\footnotetext{
${ }^{14}$ This is because the opposite sign was used in $[12,36]$ for the definition of $R_{\alpha}$.
} 
with the symmetry-adapted SH frames of Bianchi type VIII and IX, which are historically the prime examples of "Mixmaster" dynamics.

The asymptotic causal structure associated with asymptotic silence indicates that local and quasi-local aspects, and not global ones, are most important when trying to understand the physics of generic spacelike singularities in GR. Since this suggests that there is no preferred choice of frame, it is natural that results should be translated to a frame-independent description in order to separate gauge features from physics. In particular, the attractor should be characterized within the framework of a spatial frame-invariant description of the union of the silent Kasner and Bianchi type II subsets; it follows from this that we are interested in quantities that do not involve $R_{\alpha}$.

Let us therefore characterize this subset in a frame-invariant way. It is described by the equations

$$
G^{i j}=\dot{U}^{2}=r^{2}=A^{2}=N_{\alpha \beta} \Sigma_{\gamma \sigma} \Sigma^{\beta \sigma}\left(N^{\alpha \gamma}-\Sigma^{\alpha \gamma}\right)=D_{N}=\Delta_{N}=0,
$$

where we have introduced the following quantities:

$$
G^{i j}:=\delta^{\alpha \beta} E_{\alpha}{ }^{i} E_{\beta}{ }^{j}, \quad D_{N}:=\operatorname{det}\left(N_{\alpha \beta}\right), \quad \Delta_{N}:=\frac{1}{2}\left[N_{\alpha \beta} N^{\alpha \beta}-\left(N_{\alpha}{ }^{\alpha}\right)^{2}\right] .
$$

The quantity $G^{i j}$ is the conformal Hubble-normalized contravariant threemetric, ${ }^{15}$ see [24]; if $N^{\alpha \beta}$ is of rank 2, then $D_{N}=0$; if $N^{\alpha \beta}$ is of rank 1 (as it is for Bianchi type II), then $D_{N}=\Delta_{N}=0$. The quantity $N_{\alpha \beta} \Sigma_{\gamma \sigma} \Sigma^{\beta \sigma}$ $\left(N^{\alpha \gamma}-\Sigma^{\alpha \gamma}\right)$ is the square of the expression $\epsilon_{\alpha}^{\beta \gamma} N_{\beta \delta} \Sigma_{\gamma}{ }^{\delta}$, which is associated with the asymptotic Codazzi constraints.

To explore the frame-invariant description of the attractor, it is of interest to consider the following Weyl spatial and spacetime scalar invariants

$$
\begin{array}{ll}
\mathcal{E}^{2}=\frac{1}{6} \mathcal{E}_{\alpha \beta} \mathcal{E}^{\alpha \beta}, & \mathcal{H}^{2}=\frac{1}{6} \mathcal{H}_{\alpha \beta} \mathcal{H}^{\alpha \beta}, \\
\mathcal{W}_{1}=\frac{C_{a b c d} C^{a b c d}}{48 H^{4}}=\mathcal{E}^{2}-\mathcal{H}^{2}, & \mathcal{W}_{2}=\frac{C_{a b c d}{ }^{a} C^{a b c d}}{48 H^{4}}=\frac{1}{3} \mathcal{E}_{\alpha \beta} \mathcal{H}^{\alpha \beta},
\end{array}
$$

where $\left\{\mathcal{E}_{\alpha \beta}, \mathcal{H}_{\alpha \beta}\right\}=\left\{E_{\alpha \beta}, H_{\alpha \beta}\right\} / H^{2}$ are the electric and the magnetic part of the Weyl curvature tensor. (Here, $E_{\alpha \beta}, H_{\alpha \beta}$ refer to the orthonormal frame components of the Weyl tensor of $\mathbf{g}$ while $\mathcal{E}_{\alpha \beta}, \mathcal{H}_{\alpha \beta}$ are the orthonormal frame components of the Weyl tensor of $\mathbf{G}$, or, equivalently, the conformal orthonormal frame components of the Weyl tensor of $\mathbf{g}$ ).

\footnotetext{
${ }^{15}$ Incidentally, this suggests that it is this object that is natural to use if one wants to pursue a metric approach to the Einstein field equations and generic spacelike singularities.
} 
The attractor consists of a sequence of Kasner states connected by Bianchi type II solutions (i.e., attractor sequences). To formulate this in terms of curvature frame invariants, we proceed as follows: we first give the electric and magnetic parts of the Weyl tensor for the $\mathcal{B}_{N_{1}}$ subset:

$$
\begin{array}{ll}
\mathcal{E}_{11}=2+\left(1-\Sigma_{1}\right) \Sigma_{1}-\frac{10}{9}\left(1-\Sigma^{2}\right), & \mathcal{H}_{11}=-\frac{3}{2} N_{1} \Sigma_{1}, \\
\mathcal{E}_{22}=2+\left(1-\Sigma_{2}\right) \Sigma_{2}-\frac{22}{9}\left(1-\Sigma^{2}\right), & \mathcal{H}_{22}=\frac{1}{2} N_{1}\left(\Sigma_{2}+2 \Sigma_{1}\right), \\
\mathcal{E}_{33}=2+\left(1-\Sigma_{3}\right) \Sigma_{3}-\frac{22}{9}\left(1-\Sigma^{2}\right), & \mathcal{H}_{33}=\frac{1}{2} N_{1}\left(\Sigma_{3}+2 \Sigma_{1}\right) .
\end{array}
$$

The equations (6.3) then allow us to express the above equations in terms of the frame-invariant $\zeta$, and subsequently we can compute the curvature scalars in terms of $\zeta$, i.e., we obtain a frame-invariant description of the scalars. However, the resulting expressions are quite complicated and we will refrain from giving them explicitly. The Kasner states yield $\mathcal{W}_{1}=27 u^{2}(1+$ $u)^{2} /\left(1+u+u^{2}\right)^{3}$ and $\mathcal{W}_{2}=0 ; \mathcal{W}_{1}$ is monotonically decreasing in $u$ with the range $(0,4)$ with $\mathcal{W}_{1}=0$ when $u=\infty$, i.e., for the Taub state, while $\mathcal{W}_{1}$ reaches its maximum value $\mathcal{W}_{1}=4$ for the non-flat LRS Kasner state $u=1$.

The scalars can be expressed implicitly in $\tau$-time for a Bianchi type II transition since it is possible to integrate (6.3); we obtain

$$
\tau(\zeta)=\ln \left[\left(\zeta-\zeta_{-}\right)^{1 / 4 u \zeta_{-}}\left(\zeta_{+}-\zeta\right)^{-1 / 4 u \zeta_{+}} \zeta^{-1 / 6}\right] .
$$

In figure $9(\mathrm{a})$, we plot a typical attractor sequence; note that a change of era occurs. We then plot the $\mathcal{E}^{2}, \mathcal{H}^{2}$ and $\mathcal{W}_{1}, \mathcal{W}_{2}$ scalars against each other in figures $9(\mathrm{~b})$ and (c), and obtain a frame-independent description of the same orbit in terms of curvature properties.

Apart from spatial gauge considerations, it is of interest to ask oneself: to what degree do asymptotic silence and local dynamics for a generic spacelike singularity depend on the temporal gauge one is using? Note that asymptotic silence has been defined gauge invariantly, and hence the issue is whether a generic spacelike singularity is asymptotically silent (which our results indicate) and to what extent asymptotic local dynamics is a temporally gauge robust feature for a generic spacelike singularity. The asymptotic causal structure associated with asymptotic silence suggests considerable temporal gauge robustness, and we expect a large class of time choices to be compatible with asymptotic local dynamics.

We first note that if one considers a foliation associated with a time choice, and if it is possible to choose a parameterization of this foliation so that $\mathcal{N}$ is bounded and greater (or smaller) than zero asymptotically toward the singularity, then the billiard attractor is a local attractor in the full 


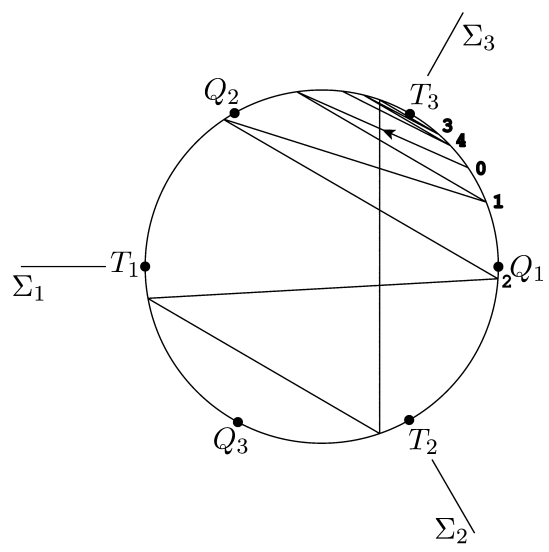

(a) A typical attractor sequence.

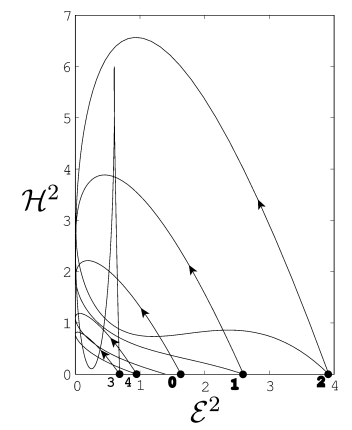

(b) $\mathcal{E}^{2}-\mathcal{H}^{2}$

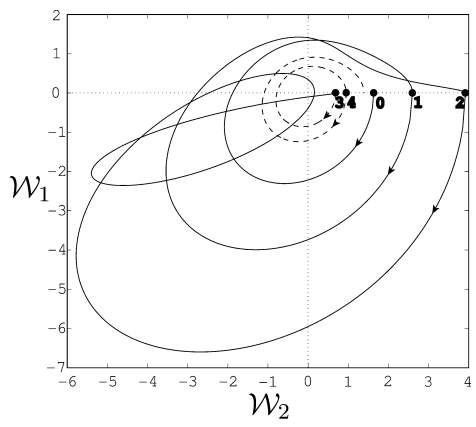

(c) $\mathcal{W}_{1}-\mathcal{W}_{2}$

Figure 9: (a) A typical attractor sequence is shown, which is associated with sequence of Kasner epoch characterized by the values of $u$ given by $\left\{u_{0}, u_{1}, u_{2}, u_{3}, u_{4}\right\}=\left\{3.18,2.18,1.18,(1.18-1)^{-1}=5.56,4.56\right\}$. (b) and (c) The corresponding Weyl scalar properties (note that the scalars are constant during frame transitions are shown); the bullets pinpoint the Kasner epochs. The dashed line in (c) indicates a new era and the arrows show the direction of increasing time toward the singularity.

state space; the difference between using such a time variable and the local reparameterization that uses $\tau$ only amounts to an unimportant conformal factor on the right hand sides of the billiard equations for each fixed $x^{i}$. We then note that the billiard attractor is a local attractor if one uses any temporal choice compatible with the asymptotic local dynamics condition, which, in particular, implies that the timelike congruence is asymptotically conformally geodesic since $\dot{U}_{\alpha} \rightarrow 0$ (which includes the synchronous choice used by BKL). However, presumably it is possible to choose a time gauge such that, e.g., $\dot{U}_{\alpha} \nrightarrow \rightarrow 0$, which is hence non-compatible with asymptotic local dynamics, even though the spacetime may exhibit a singularity for 
which there exists a large class of time choices for which the dynamics is asymptotically local.

\section{Concluding remarks}

In this paper, we have considered the dynamics of solutions of the Einstein equations in the asymptotic limit toward a generic spacelike singularity: the asymptotic behavior of solutions is represented by the billiard attractor and the associated attractor sequences. We have derived the billiard attractor based on the dynamical systems formulation of the Einstein equations in the conformal Hubble-normalized Iwasawa frame approach; in addition, we have established the "duality" of the presented framework with the Hamiltonian approach to cosmological billiards of Damour et al. [13].

The cornerstones of our derivation of the billiard attractor have been (i) the identification of a hierarchy of state spaces and invariant subsets; in the asymptotic limit, dynamics is restricted to subsets of subsets, to boundaries of boundaries, descending from the full state space via the SH silent boundary and the oscillatory subset down to the billiard attractor; (ii) a thorough analysis of sequences of transitions; in particular, we have introduced the concepts of small and large curvature phases that appeared prominently in our considerations; (iii) stochastic analysis; we have investigated the probabilistic nature of Kasner sequences and we have discussed the effects leading to randomization; randomized Kasner sequences and randomized asymptotic attractor sequences are fundamental concepts in our analysis; (iv) the computation of decay rates; in connection with probabilistic aspects, we have given the decay rates of several quantities; this decay has turned out to be the underlying reason for the restriction of the dynamics to subsets and boundaries of subsets. The presented derivation of the billiard conjecture is not mathematically rigorous, but depends on arguments that are merely heuristic - despite their being convincing. However, we are positive that in any endeavor aimed at obtaining proofs, several concepts and methods introduced in this paper will play a prominent role. Let us elaborate on this.

Generalizing the concept of an era has played an important role in our analysis. The partitioning of sequences into small and large curvature phases has shown that small curvature phases dominate over large curvature phases from a stochastic point of view; hence, in the asymptotic evolution of solutions, the phases when the solution is close to the Taub solution are crucial a fact that might not have been appreciated enough previously. Note that the division into small and large curvature phases has featured prominently in the more technical computations, see Appendix F for details. 
Asymptotic (attractor) sequences give a precise meaning to the notion of "piece-wise approximations" by BKL. The dynamics of a generic timeline of a solution with a generic spacelike singularity is expected to asymptotically shadow the attractor, and hence the associated orbit can be partitioned into segments where each segment can be approximated with an increasing degree of accuracy by a heteroclinic orbit on the attractor. However, due to "errors" associated with the approach toward the attractor, the solution does not exactly follow the heteroclinic orbit structure on the attractor; instead these increasingly small errors lead to an approximation that might be subsumed under the notion of a randomized sequence of heteroclinic orbits.

Randomization constitutes an important ingredient in several contexts. The underlying structures that allowed one to obtain mathematical proofs about the attractor of the diagonal Bianchi type IX models in a Fermipropagated frame $[18,19]$ are specific for these models and are not available in other cases; since it is such cases that are relevant for the present general scenario, the diagonal Bianchi type IX models are misleading. Our present analysis suggests that one has to know the (asymptotic) history of a solution to unravel its asymptotic features; this causes a dilemma since this requires that one finds the solution, which seems unlikely. However, randomization makes it possible to stochastically examine the cumulative effects of small and large curvature phases, and this allows one to estimate decay rates and give a description of what is going to happen generically. In our opinion, statistical analysis will be an essential new ingredient in future proofs.

In this paper, we have obtained decay rates associated with asymptotic suppression and freezing (see Appendix $\mathrm{H}$ ), which will hold in the neighborhood of the billiard attractor as well. Since decay rates are likely to be ingredients in any proof, it is important that numerics not only check that the dynamics of a generic spatial point approaches the attractor, but that the attractor is approached in the way described by the decay rates; therefore, these results offer new input for numerical investigations.

As we have seen, the asymptotic dynamics is inevitably restricted to subsets of subsets, to boundaries of boundaries, which underlines the importance of the hierarchical structure of invariant sets associated with asymptotically local SH dynamics. However, there also exists a hierarchy of invariant subsets that is based on the number of Killing vectors the spacetime admits. This symmetry-based hierarchy is of interest since it is associated with different levels of technical difficulty; taking highly symmetric, and thus technically simple, cases as a starting point, one can analytically and numerically explore, step-by-step, the cases of decreasing symmetry by making use of the gained experience; since several structures that are relevant to the non-symmetric case already appear in cases of special symmetries, this is 
likely to lead the way to the generic case. The present work shows how the Hubble-normalized Iwasawa frame approach naturally provides a framework for dealing with this type of hierarchy; the formulation comprises different symmetry levels; note, however, that the Iwasawa decomposition is natural only when one has commuting spacelike Killing vectors, and hence it is not appropriate for Bianchi types VIII or IX. The simplest model with an oscillatory singularity as described by the present formalism is the general Bianchi type $\mathrm{VI}_{-1 / 9}$ model. The next level of difficulty as regards oscillatory singularities is the general case with two commuting spacelike Killing vectors; then the general case with one spacelike Killing vector; and finally the general case with no Killing vectors. Incidentally, by imposing geometric restrictions on the Killing vectors such as hypersurface orthogonality, see, e.g., $[12,45]$, one can obtain relatively simple classes of models with nonoscillatory singularities. Exploiting this outlined hierarchy of symmetries might prove to be the key to a complete understanding of the nature of generic spacelike singularities.

A dynamical system that is of particular interest in our analysis is the system (11.1); when we do not impose the Codazzi constraint (11.1h), we refer to this system as the unconstrained billiard system, see also the discussion in Section 12 in connection with the "dominant" Hamiltonian. We expect the unconstrained billiard system to possess the same generic asymptotic behavior as the full system $\boldsymbol{X}$ in the neighborhood of an asymptotically silent singularity; hence, in our approach, it is this system that is of central importance for the asymptotic dynamics, rather than, e.g., the Bianchi type IX system. We will provide a thorough analysis of this system and its properties elsewhere.

Another interesting issue we have put little emphasis on in the present paper is the question of consistency. It is suggestive to take the billiard attractor and the associated billiard conjecture as a starting point for further developments by inserting attractor sequences $\mathcal{A}_{\mathcal{T}}$ into the relevant equations; using similar reasoning as done here for the suppression of variables, we will be able to derive more substantial justification for the claim that $E_{\alpha}{ }^{i} \rightarrow 0$ (a major ingredient for asymptotic silence) and $\boldsymbol{S}_{\text {stable }} \rightarrow 0$ when $\tau \rightarrow \infty$ (cf. steps A and B in Section 11). In addition, we will obtain further information on the approach of solutions toward the asymptotic limit. We will pursue issues of this kind in future work.

In this paper, we have been concerned with the vacuum GR case, however, the approach and methods we have developed will be applicable to the case when matter is included and also to other theories (see, e.g., $[13,14]$ and references therein). As regards possible sources, this naturally suggests a study of the influence of matter on singularities. As a simple example 
we refer to [46], which gives an indication that, e.g., Vlasov matter behaves differently in some respects than perfect fluids. Of particular interest is the question of structural stability of generic spacelike singularities, especially since generic singularities seem to uncover the essential properties of matter. There are indications that a classification of the influence of matter on generic singularity structure naturally rests on (i) energy conditions and (ii) whether the effective propagation speed is less than the speed of light or not. There are also indications that suggest a subclassification based on the behavior of matter in the case of speed of light propagation: e.g., massless scalar fields and electromagnetic fields influence the generic spacelike singularity in different ways; does this motivate a subclassification based on spin? In the sublight case, it seems to be natural to base a subclassification on the question of what features are affected by matter and what features are not, e.g., the Hubble-normalized energy-momentum tensor may go to zero, which leads to an asymptotic description of the geometry by vacuum solutions, but this does not necessarily mean that, e.g., the Hubble-normalized rotation of a perfect fluid tends to zero. Another issue is how, and if, matter influences the connection between generic spacelike singularities and weak null singularities in asymptotically flat spacetimes, see [23]. Questions and issues like these lead to a variety of possible research projects that could make our understanding more substantial.

In this paper, we have assumed asymptotic silence and asymptotic local dynamics, and our work has provided results and evidence that support the consistency of this scenario for generic timelines. However, this does not mean that there could not exist interesting phenomena associated with special timelines, indeed, we believe that a set of measure zero of timelines exhibit spike formation and recurring "spike transitions" [22] that are associated with non-local dynamics. Even so, it still seems that asymptotic silence prevails and that asymptotic local dynamics play an important role - remarkably, spike transitions seem to be governed by variations of the Kasner map, which is associated with asymptotically local dynamics, hinting at further more deeply hidden structures. Asymptotic spike formation is associated with the unstable variables $N_{1}, R_{1}, R_{3}$ going through a zero at a spatial point (where a zero in $N_{1}$ yields a "true spike", seen in the curvature, while zeroes in $R_{1}, R_{3}$ yield false spikes, i.e., gauge effects not seen in the curvature; presumably, zeroes in $N_{2}$ and $R_{2}$ do not play a similar role since these variables are generically suppressed in the asymptotic limit). There are hints that spikes may interfere - destructively and constructively. Although uncertain, constructive spike interference seems to dominate, which, if correct, leads to asymptotic "spike cascading" W.C. Lim, Private Communication. There are many other unresolved spike issues as well: are there spikes that undergo infinitely many recurring spike transitions? Where do 
spikes form and in what way - how does spike interference work? Do spikes asymptotically form a dense set? Can spikes leave observational imprints in, e.g., the cosmic microwave background? These question are clearly interesting in themselves, moreover, a clarification of some of these issues is of considerable interest in the context of eventual generic singularity proofs.

Ultimately one might ask oneself the question why generic singularities should be studied at all in a classical GR context? Firstly, there exists a regime between the Planck era and the GUT era where GR is expected to hold and where the approach toward the singularity is presumably described by the dynamics toward a generic singularity (recall that one of the points of inflation is to "erase the effects of initial data" and that before this erasure a singularity is presumably generic according to this line of reasoning). Secondly, black hole formation is associated with initial data reflecting the complexities of the real universe; one would hence also in this case expect generic spacelike singularities to play a role before one enters the Planck regime. Thirdly, the formation of generic singularities is associated with considerable structure, even in the case of spike formation: can this structure be used to asymptotically quantize gravity where it needs to be quantized, namely in the ultra-strong gravitational field in the neighborhood of a generic spacelike singularity? ${ }^{16}$

\section{Acknowledgments}

It is a pleasure to thank Lars Andersson, Henk van Elst, Woei Chet Lim, and John Wainwright for many helpful and stimulating discussions. C.U. is supported by the Swedish Research Council.

\section{Appendices}

\section{A Iwasawa variables and useful equations}

In this section, we derive in some detail the connection between the Hamiltonian Iwasawa variables of Section 2 and the conformal Hubble-normalized

\footnotetext{
${ }^{16}$ For the exploitation of some of these structures in the context of quantization of special models, see, e.g., $[47,48]$.
} 
variables of Section 3. Let us begin by recalling that for an Iwasawa frame

$$
\left.e_{i}^{\alpha}=\exp \left(-b^{\alpha}\right) \mathcal{N}^{\alpha}{ }_{i}, \quad e_{\alpha}{ }^{i}=\exp \left(b^{\alpha}\right) \overline{\mathcal{N}}^{i}{ }_{\alpha} \quad \text { (no summation over } \alpha\right),
$$

where $\mathcal{N}^{\alpha}{ }_{i}$ and $\overline{\mathcal{N}}^{i}{ }_{\alpha}$ are the upper triangular matrices given by (2.5) and (2.6), respectively; the off-diagonal components of $\mathcal{N}^{\alpha}{ }_{i}\left(\overline{\mathcal{N}}^{i}{ }_{\alpha}\right)$ are denoted by $n_{\alpha}\left(\bar{n}_{\alpha}\right)$. Therefore, due to (3.3), we obtain for the conformal frame components:

$$
E_{i}^{\alpha}=H \exp \left(-b^{\alpha}\right) \mathcal{N}^{\alpha}{ }_{i}, \quad E_{\alpha}{ }^{i}=H^{-1} \exp \left(b^{\alpha}\right) \overline{\mathcal{N}}^{i}{ }_{\alpha} \quad \text { (no summation). }
$$

In the following, we use the notation $E_{\alpha}=E_{\alpha}{ }^{\alpha}$ (no sum over $\alpha$ ) and analogously $e_{\alpha}=e_{\alpha}{ }^{\alpha}$. Note that the frame variables $e_{\alpha}$ have been chosen to be positive, from which it follows that also $E_{\alpha}>0$, since we take the cosmological model to be expanding toward the future and hence $H>0$. Equation (A.1) can be inverted easily:

$$
b^{\alpha}=\log \left(e_{\alpha}\right)=\log \left(H E_{\alpha}\right), \quad \bar{n}_{1}=\frac{E_{2}{ }^{1}}{E_{2}}, \quad \bar{n}_{2}=\frac{E_{3}{ }^{1}}{E_{3}}, \quad \bar{n}_{3}=\frac{E_{3}{ }^{2}}{E_{3}} .
$$

In order to make contact between the Hamiltonian variables and the conformal Hubble-normalized variables, we first consider the Hubble scalar $H$. Since $g_{i j}=e_{i}^{\alpha} e^{\beta}{ }_{j} \delta_{\alpha \beta}$, we have

$$
\sqrt{g}=\exp \left(-b^{1}-b^{2}-b^{3}\right)=\exp \left(-\sum_{\alpha} b^{\alpha}\right) ;
$$

accordingly, we obtain for the derivative (w.r.t. coordinate time $x^{0}$ ):

$$
\frac{\partial}{\partial x^{0}} \sqrt{g}=-\sqrt{g}\left(\frac{\partial}{\partial x^{0}} \sum_{\alpha} b^{\alpha}\right) .
$$

Since the derivative of $\sqrt{g}$ is proportional to the expansion $\theta$ according to

$$
\frac{1}{\sqrt{g}} \frac{\partial}{\partial x^{0}} \sqrt{g}=N \theta=3 N H
$$

this implies that

$$
H=-\frac{1}{3} \frac{1}{N} \frac{\partial}{\partial x^{0}} \sum_{\alpha} b^{\alpha}
$$


Using the relation (2.9a) between $\dot{b}^{\alpha}$ and $\pi_{\alpha}$, where we recall that $N=$ $\tilde{N} \sqrt{g}$, we obtain a representation of $H$ in terms of the momenta, i.e.,

$$
H=\frac{1}{12} \frac{1}{\sqrt{g}} \sum_{\alpha} \pi_{\alpha}=\frac{1}{12}\left(\exp \sum_{\alpha} b^{\alpha}\right) \sum_{\beta} \pi_{\beta} .
$$

Equation (A.5) suggests that we define a quantity $\Lambda$,

$$
\Lambda:=2 H \sqrt{g}=\frac{1}{6} \sum_{\alpha} \pi_{\alpha},
$$

which we will use frequently in the following. In terms of the frame components, $\Lambda$ can be expressed as

$$
\Lambda=2 H \sqrt{g}=2\left(H^{2} E_{1} E_{2} E_{3}\right)^{-1},
$$

since $\sqrt{g}=\left(e_{1} e_{2} e_{3}\right)^{-1}=H^{-3}\left(E_{1} E_{2} E_{3}\right)^{-1}$.

Inverting the commutator equations (3.4) yields the conformal Hubblenormalized variables in terms of $\mathcal{N}$ and $E_{\alpha}{ }^{i}$ and their derivatives:

$$
\begin{aligned}
& \dot{U}_{\alpha}=\boldsymbol{\partial}_{\alpha} \log \mathcal{N}, \quad q=\frac{1}{3} E^{\alpha}{ }_{i} \partial_{0} E_{\alpha}{ }^{i}, \\
& R^{\alpha}=\frac{1}{2} \epsilon^{\alpha}{ }_{\beta}^{\gamma} E^{\beta}{ }_{i} \partial_{0} E_{\gamma}{ }^{i}, \quad \Sigma_{\alpha \beta}=-E^{\gamma}{ }_{i} \boldsymbol{\partial}_{0} E_{\langle\alpha}{ }^{i} \delta_{\beta\rangle \gamma}, \\
& A_{\alpha}=\frac{1}{2} E^{\beta}{ }_{i} \boldsymbol{\partial}_{\alpha} E_{\beta}{ }^{i}-\frac{1}{2} E^{\beta}{ }_{i} \boldsymbol{\partial}_{\beta} E_{\alpha}{ }^{i}, \quad N^{\alpha \beta}=E^{(\alpha}{ }_{i} \epsilon^{\beta) \gamma \delta} \boldsymbol{\partial}_{\gamma} E_{\delta}^{i} .
\end{aligned}
$$

Inserting (A.2) into equation (A.8a) for $q$ yields

$$
q=-\frac{\partial_{0} H}{H}+\frac{1}{3} \partial_{0} \sum_{\alpha} b^{\alpha}
$$

Since $\partial_{x^{0}}=H N \boldsymbol{\partial}_{0}$, equation (A.4) takes the simple form $\boldsymbol{\partial}_{0} \sum_{\alpha} b^{\alpha}=-3$, which in turn implies $\boldsymbol{\partial}_{0} \sqrt{g}=3 \sqrt{g}$ from (A.3). Using the expression (A.5) to compute $\partial_{0} H$, we eventually obtain

$$
q=2-\frac{1}{6} \Lambda^{-1} \partial_{0} \sum_{\alpha} \pi_{\alpha}
$$

Note that equation (A.9) reproduces the dimensional equation $\partial_{0} H=$ $-(1+q) H$ for $H$. 
Inserting (A.2) into (A.8b) yields

$$
\begin{aligned}
R_{1} & =-\frac{1}{2} e^{b^{3}-b^{2}} \boldsymbol{\partial}_{0} n_{3}, \quad R_{2}=\frac{1}{2} e^{b^{3}-b^{1}}\left(-n_{3} \boldsymbol{\partial}_{0} n_{1}+\boldsymbol{\partial}_{0} n_{2}\right), \\
R_{3} & =-\frac{1}{2} e^{b^{2}-b^{1}} \boldsymbol{\partial}_{0} n_{1}, \\
\Sigma_{23} & =+\frac{1}{2} e^{b^{3}-b^{2}} \boldsymbol{\partial}_{0} n_{3}, \quad \Sigma_{13}=\frac{1}{2} e^{b^{3}-b^{1}}\left(-n_{3} \boldsymbol{\partial}_{0} n_{1}+\boldsymbol{\partial}_{0} n_{2}\right), \\
\Sigma_{12} & =+\frac{1}{2} e^{b^{2}-b^{1}} \boldsymbol{\partial}_{0} n_{1}
\end{aligned}
$$

for $R_{\alpha}$ and $\Sigma_{\alpha \beta}(\alpha \neq \beta)$, and

$$
\begin{aligned}
& \Sigma_{11}=\frac{1}{3} \boldsymbol{\partial}_{0} \sum_{\alpha} b^{\alpha}-\boldsymbol{\partial}_{0} b^{1}, \quad \Sigma_{22}=\frac{1}{3} \boldsymbol{\partial}_{0} \sum_{\alpha} b^{\alpha}-\boldsymbol{\partial}_{0} b^{2}, \\
& \Sigma_{33}=\frac{1}{3} \boldsymbol{\partial}_{0} \sum_{\alpha} b^{\alpha}-\boldsymbol{\partial}_{0} b^{3}
\end{aligned}
$$

for the diagonal elements of $\Sigma_{\alpha \beta}$. The main observation is that the offdiagonal components of $\Sigma_{\alpha \beta}$ are given by the Fermi rotation parameters $R_{\alpha}$ according to

$$
\left(\Sigma_{23}, \Sigma_{31}, \Sigma_{12}\right)=\left(-R_{1}, R_{2},-R_{3}\right),
$$

which is a fundamental property of the Iwasawa gauge.

In order to express to r.h.s. of (A.11) in terms of the momenta $\pi_{\alpha}$ and $\mathcal{P}_{\alpha}$, we write (2.9) in the form

$$
\begin{aligned}
& \boldsymbol{\partial}_{0} b^{\alpha}=\frac{1}{\Lambda} \sum_{\beta} \mathcal{G}^{\alpha \beta} \pi_{\beta}, \quad \boldsymbol{\partial}_{0} n_{2}=\frac{2}{\Lambda} e^{2\left(b^{1}-b^{2}\right)}\left(n_{3} \mathcal{P}_{1}+\left(e^{2\left(b^{2}-b^{3}\right)}+n_{3}^{2}\right) \mathcal{P}_{2}\right) \\
& \boldsymbol{\partial}_{0} n_{1}=\frac{2}{\Lambda} e^{2\left(b^{1}-b^{2}\right)}\left(\mathcal{P}_{1}+n_{3} \mathcal{P}_{2}\right), \quad \boldsymbol{\partial}_{0} n_{3}=\frac{2}{\Lambda} e^{2\left(b^{2}-b^{3}\right)} \mathcal{P}_{3},
\end{aligned}
$$

where we use $\tilde{N}^{-1} \partial_{x^{0}}=(\Lambda / 2) \partial_{0}$. A straightforward computation then results in

$$
\begin{aligned}
& \Sigma_{\alpha}=\Sigma_{\alpha \alpha}=2-\Lambda^{-1} \pi_{\alpha}, \quad(\text { no sum }) \\
& R_{1}=-\Lambda^{-1} \exp \left(b^{2}-b^{3}\right) \mathcal{P}_{3}, \quad R_{2}=\Lambda^{-1} \exp \left(b^{1}-b^{3}\right) \mathcal{P}_{2} \\
& R_{3}=-\Lambda^{-1} \exp \left(b^{1}-b^{2}\right)\left(n_{3} \mathcal{P}_{2}+\mathcal{P}_{1}\right)
\end{aligned}
$$

where $\Lambda$ is given in terms of the Hamiltonian variables by (A.6). 
Inversion of (A.14) yields

$$
\begin{aligned}
& \pi_{\alpha}=\Lambda\left[2-\Sigma_{\alpha}\right], \\
& \mathcal{P}_{1}=-\Lambda R_{3} E_{2} / E_{1}+\bar{n}_{3} \mathcal{P}_{2}, \quad \mathcal{P}_{2}=\Lambda R_{2} E_{3} / E_{1}, \\
& \mathcal{P}_{3}=-\Lambda R_{1} E_{3} / E_{2},
\end{aligned}
$$

where $\Lambda$ is given by (A.7).

To obtain the conformal Hubble-normalized variables $A_{\alpha}$ and $N_{\alpha \beta}$ in terms of the Hamiltonian variables, we proceed analogously. Based on (A.8c), we find

$$
\begin{aligned}
N_{\alpha} & =\Lambda^{-1} \exp \left(-2 b^{\alpha}\right) \tilde{N}_{\alpha} \quad(\text { no summation over } \alpha), \\
N_{\alpha \beta} & =\Lambda^{-1} \exp \left(-\left(b^{\alpha}+b^{\beta}\right)\right) f_{\gamma-} \quad(\alpha \neq \beta \neq \gamma), \\
A_{\alpha}-r_{\alpha} & =\Lambda^{-1} \exp \left(-\left(b^{\alpha}+b^{\beta}\right)\right) f_{\gamma+} \quad(\alpha \neq \beta \neq \gamma),
\end{aligned}
$$

where

$$
\begin{aligned}
\tilde{N}_{1} & =2\left(\bar{\partial}_{2} \bar{n}_{3}-\bar{\partial}_{3} \bar{n}_{1}+\left(\bar{n}_{1}+1\right)\left(\bar{n}_{1} \bar{\partial}_{1} \bar{n}_{3}-\bar{\partial}_{2} \bar{n}_{3}\right)\right) \\
\tilde{N}_{2} & =-2 \bar{\partial}_{1} \bar{n}_{3}, \\
\tilde{N}_{3} & =0, \\
f_{1 \pm} & =\bar{\partial}_{1}\left(b^{3} \pm b^{2}\right), \quad \bar{\partial}_{1}=\partial_{1}, \\
f_{2 \pm} & =\bar{\partial}_{2}\left(b^{3} \pm b^{1}\right) \mp \bar{\partial}_{1} \bar{n}_{1}, \quad \bar{\partial}_{2}=\bar{n}_{1} \partial_{1}+\partial_{2}, \\
f_{3 \pm} & =\bar{\partial}_{3}\left(b^{1} \pm b^{2}\right) \mp \bar{\partial}_{2} \bar{n}_{3}+\bar{n}_{1} \bar{\partial}_{1} \bar{n}_{3}-\bar{\partial}_{1} \bar{n}_{2}, \quad \bar{\partial}_{3}=\bar{n}_{2} \partial_{1}+\bar{n}_{3} \partial_{2}+\partial_{3} ;
\end{aligned}
$$

as usual we denote the diagonal elements of $N_{\alpha \beta}$ by $N_{\alpha}$. In particular we see that

$$
N_{3}=0
$$

in the Iwasawa gauge.

Finally, we note that the momentum conjugate to $\lambda=\log \rho$ reads

$$
\pi_{\lambda}=\Lambda \log \left[\left(e_{1}\right)^{\left(2-\Sigma_{1}\right)}\left(e_{2}\right)^{\left(2-\Sigma_{2}\right)}\left(e_{3}\right)^{\left(2-\Sigma_{3}\right)}\right] .
$$

where $e_{\alpha}=H E_{\alpha}=\exp \left(b^{\alpha}\right)$.

We conclude this section by investigating the equations for the conformal spatial frame components $E_{\alpha}{ }^{i}$ and the consequences thereof in some detail; 
these equations will be useful in Appendix H. From (3.4a) and (3.7a), we see

$$
\partial_{0} E_{\alpha}{ }^{i}=F_{\alpha}{ }^{\beta} E_{\beta}{ }^{i}, \quad \text { where } F_{\alpha}{ }^{\beta}=q \delta_{\alpha}^{\beta}-\Sigma_{\alpha}^{\beta}-\epsilon_{\alpha \gamma}{ }^{\beta} R^{\gamma} .
$$

In Iwasawa gauge (A.12), we obtain

$$
\partial_{0} E_{1}=\left(q-\Sigma_{1}\right) E_{1}, \quad \partial_{0} E_{2}=\left(q-\Sigma_{2}\right) E_{2}, \quad \partial_{0} E_{3}=\left(q-\Sigma_{3}\right) E_{3},
$$

where $E_{1}, E_{2}, E_{3}$, and $\Sigma_{1}, \Sigma_{2}, \Sigma_{3}$, are again the diagonal components of $E_{\alpha}{ }^{i}$ and $\Sigma_{\alpha \beta}$, and

$$
\begin{aligned}
& \partial_{0} E_{2}{ }^{1}=2 R_{3} E_{1}+\left(q-\Sigma_{2}\right) E_{2}{ }^{1}, \quad \partial_{0} E_{3}{ }^{2}=2 R_{1} E_{2}+\left(q-\Sigma_{3}\right) E_{3}{ }^{1}, \\
& \partial_{0} E_{3}{ }^{1}=-2 R_{2} E_{1}+2 R_{1} E_{2}{ }^{1}+\left(q-\Sigma_{3}\right) E_{3}{ }^{1}
\end{aligned}
$$

for the non-zero off-diagonal components of $E_{\alpha}{ }^{i}$. Using that

$$
\partial_{0} H=-(1+q) H,
$$

we find for the derivatives of $e_{\alpha}=H E_{\alpha}$ :

$$
\partial_{0} e_{1}=-\left(1+\Sigma_{1}\right) e_{1}, \quad \partial_{0} e_{2}=-\left(1+\Sigma_{2}\right) e_{2}, \quad \partial_{0} e_{3}=-\left(1+\Sigma_{3}\right) e_{3} .
$$

Furthermore, from

$$
\sqrt{g}=H^{-3}\left(E_{1} E_{2} E_{3}\right)^{-1}
$$

we obtain

$$
\partial_{0} \sqrt{g}=3 \sqrt{g}
$$

from a direct computation based on (A.17a); therefore,

$$
\partial_{0} \Lambda=\partial_{0}(2 H \sqrt{g})=(2-q)(2 H \sqrt{g})=(2-q) \Lambda .
$$

Evidently, this equation is consistent with (A.6) and (A.10).

\section{B Deriving the generalized Kasner line element of BKL}

The Kasner subset on the silent boundary $E_{\alpha}{ }^{i}=0$ is characterized by $\dot{U}_{\alpha}=$ $r_{\alpha}=0$ (SH condition) and $0=1-\Sigma^{2}=A_{\alpha}=N_{\alpha \beta}$. We specialize to the Kasner circle $\mathrm{K}^{\bigcirc}$ by setting $R_{\alpha}=0$, which corresponds to setting $\Sigma_{\alpha \beta}=0$ $(\alpha \neq \beta)$. Therefore, for every point on $\mathrm{K}^{\bigcirc}, \Sigma_{\alpha \beta}=\operatorname{diag}\left[\hat{\Sigma}_{1}, \hat{\Sigma}_{2}, \hat{\Sigma}_{3}\right]$, where 
the shear variables $\hat{\Sigma}_{\alpha}(\alpha=1, \ldots, 3)$ are temporally constant spatially dependent functions; we set $\operatorname{diag}\left[\hat{\Sigma}_{11}, \hat{\Sigma}_{22}, \hat{\Sigma}_{33}\right]=\operatorname{diag}\left[3 p_{1}-1,3 p_{2}-1,3 p_{3}-1\right]$.

In order to derive the leading asymptotic expressions for $H$ and $E_{\alpha}{ }^{i}$, we first note that $q=2$, which is due to (3.9a). Equations (3.5) and (3.4a), (3.7a) yield

$$
H=\hat{H} e^{3 \tau}, \quad E_{\alpha}{ }^{i}=\hat{E}_{\alpha}{ }^{i} e^{-3\left(1-p_{\alpha}\right) \tau} \quad(\text { no sum over } \alpha),
$$

which leads to

$$
e^{\alpha}{ }_{i}=\hat{e}^{\alpha}{ }_{i} e^{-3 p_{\alpha} \tau} \quad(\text { no sum over } \alpha) ;
$$

recall that hatted objects denote temporally constant spatially dependent functions.

In the synchronous gauge of BKL, i.e., $N=1, \mathcal{N}=H$, equation (4.3) leads to

$$
t=\hat{t}+\frac{1}{3} \hat{H}^{-1} e^{-3 \tau} .
$$

In addition, BKL also choose the synchronous time coordinate to be a simultaneous bang time function. In the present derivation, this amounts to setting $\hat{t}=0$ as an initial value condition, which can be accomplished by making a coordinate transformation. Consequently, we obtain $t \propto e^{-3 \tau}$. With suitable redefinitions, i.e., $\hat{e}_{i}{ }_{i} \propto l_{i}, \hat{e}^{2}{ }_{i} \propto m_{i}, \hat{e}^{3}{ }_{i} \propto n_{i}$, and $a=t^{p_{1}}$, $b=t^{p_{2}}, c=t^{p_{3}}$, the "generalized Kasner line element" of BKL ensues:

$$
d s^{2}=-d t^{2}+\left[a^{2} l_{i} l_{j}+b^{2} m_{i} m_{j}+c^{2} n_{i} n_{j}\right] d x^{i} d x^{j} .
$$

Thus, instead of ad hoc assuming the above line element, and inserting it into the field equations in order to analyze its consistency, we have now derived it as the lowest order perturbation of $\mathrm{K}^{\bigcirc}$ on the silent boundary.

Finally, note that an equivalent derivation can be performed in a Fermi frame instead of an Iwasawa frame: we could start with $R_{\alpha}=0$, which would lead to a 4-dimensional ellipsoid of Kasner equilibrium points characterized by temporally constant values $\hat{\Sigma}_{\alpha \beta}$, and then make a temporally constant rotation and diagonalize this matrix.

\section{Kasner solutions in a rotating frame}

It is customary to represent a generalized Kasner solution in a Fermi frame with constant diagonal shear variables: $\operatorname{diag}\left[\hat{\Sigma}_{1}, \hat{\Sigma}_{2}, \hat{\Sigma}_{3}\right]=$ const. However, 
when one considers a representation in a frame that rotates w.r.t. the Fermi frame, one obtains time-dependent non-diagonal shear variables $\Sigma_{\alpha \beta}(\tau)$ that are related to $\hat{\Sigma}_{\alpha}$ through time-dependent rotations $O_{\beta}^{\alpha}(\tau)$, i.e.,

$$
\begin{aligned}
\left(O_{\alpha^{\prime}}^{\alpha} \Sigma_{\alpha \beta} O_{\beta^{\prime}}^{\beta}\right)_{\alpha^{\prime}, \beta^{\prime}} & =\operatorname{diag}\left(\hat{\Sigma}_{1}, \hat{\Sigma}_{2}, \hat{\Sigma}_{3}\right) \\
& =\operatorname{diag}\left(3 p_{1}-1,3 p_{2}-1,3 p_{3}-1\right)=\mathrm{const}
\end{aligned}
$$

where the quantities $\left(\hat{\Sigma}_{1}, \hat{\Sigma}_{2}, \hat{\Sigma}_{3}\right)$ can be regarded as the time-independent eigenvalues of $\Sigma_{\alpha \beta}(\tau)$. Accordingly, $\Sigma_{\alpha \beta}(\tau)$ generates time-independent frame invariants: the linear invariant reduces to the trace-free shear condition, i.e., $\operatorname{tr}\left(\Sigma_{\alpha \beta}(\tau)\right)=\Sigma_{1}(\tau)+\Sigma_{2}(\tau)+\Sigma_{3}(\tau)=\hat{\Sigma}_{1}+\hat{\Sigma}_{2}+\hat{\Sigma}_{3}=0$; the quadratic invariant yields the Gauss constraint, i.e.,

$$
\Sigma^{\alpha \beta} \Sigma_{\alpha \beta}=\Sigma_{1}^{2}+\Sigma_{2}^{2}+\Sigma_{3}^{2}+2 R_{1}^{2}+2 R_{2}^{2}+2 R_{3}^{2}=\hat{\Sigma}_{1}^{2}+\hat{\Sigma}_{2}^{2}+\hat{\Sigma}_{3}^{2}=6 .
$$

Finally, the conserved cubic invariant is given by

$$
\operatorname{det} \Sigma_{\alpha \beta}(\tau)=\hat{\Sigma}_{1} \hat{\Sigma}_{2} \hat{\Sigma}_{3}=\text { const. }
$$

This invariant can also be expressed in terms of a Weyl scalar. The magnetic Weyl tensor is identically zero for Kasner, but the electric part is non-trivial and thus one obtains one non-zero quadratic Weyl scalar, related to $\operatorname{det}\left(\Sigma_{\alpha \beta}\right)$ according to

$$
\mathcal{W}_{1}=\frac{C_{a b c d} C^{a b c d}}{48 H^{4}}=2-\operatorname{det}\left(\Sigma_{\alpha \beta}\right)=\text { const. }
$$

Note that $C_{a b c d} C^{a b c d}$ is the Kretschmann scalar associated with the metric g while the quantity $C_{a b c d} C^{a b c d} / H^{4}$ is the Kretschmann scalar connected with $\mathbf{G}$.

Since $\operatorname{det} \Sigma_{\alpha \beta}=\hat{\Sigma}_{1} \hat{\Sigma}_{2} \hat{\Sigma}_{3}$ and $\left(\hat{\Sigma}_{1}, \hat{\Sigma}_{2}, \hat{\Sigma}_{3}\right) \in \mathrm{K}^{\bigcirc}$, $\operatorname{det} \Sigma_{\alpha \beta}$ is a constant in the range $[-2,2]$. Conversely, each value of $\operatorname{det} \Sigma_{\alpha \beta}$ from the interval $(-2,2)$ generates a unique ordered triple $\hat{\Sigma}_{\alpha}<\hat{\Sigma}_{\beta}<\hat{\Sigma}_{\gamma}(\alpha \neq \beta \neq \gamma \neq \alpha)$, while det $\Sigma_{\alpha \beta}= \pm 2$ leads to the LRS points $\Sigma_{\alpha}= \pm 2, \Sigma_{\beta}=\Sigma_{\gamma}=\mp 1(\alpha \neq$ $\beta \neq \gamma \neq \alpha)$.

The value of $\operatorname{det} \Sigma_{\alpha \beta}$ thus characterizes a Kasner state frame invariantly and uniquely; however, it is usually more convenient to use the Kasner 
parameter $u$ instead, which is defined implicitly through

$$
\operatorname{det}\left(\Sigma_{\alpha \beta}\right)=2-\frac{27 u^{2}(1+u)^{2}}{\left(1+u+u^{2}\right)^{3}}=\text { const, } \quad u \in[1, \infty]
$$

where $\operatorname{det} \Sigma_{\alpha \beta}$ is a monotonically increasing function of $u$. The Kasner parameter $u \in[1, \infty]$ parameterizes the one-parameter set of Kasner states $\left(p_{1}, p_{2}, p_{3}\right)$ according to

$$
p_{\alpha}=\frac{-u}{1+u+u^{2}}, \quad p_{\beta}=\frac{1+u}{1+u+u^{2}}, \quad p_{\gamma}=\frac{u(1+u)}{1+u+u^{2}}
$$

for sector $(\alpha, \beta, \gamma)$ of the Kasner circle, i.e., $p_{\alpha}<p_{\beta}<p_{\gamma}\left(\hat{\Sigma}_{\alpha}<\hat{\Sigma}_{\beta}<\hat{\Sigma}_{\gamma}\right)$; here $u \in(1, \infty)$. It is easy to check that $p_{\alpha}+p_{\beta}+p_{\gamma}=1$ and $p_{\alpha}^{2}+p_{\beta}^{2}+$ $p_{\gamma}^{2}=1$. The value $u=1$ represents the points $\mathrm{Q}_{1}, \mathrm{Q}_{2}, \mathrm{Q}_{3}$, i.e., the three equivalent representations of the non-flat LRS Kasner solution; $u=\infty$ defines the Taub points $\mathrm{T}_{1}, \mathrm{~T}_{2}, \mathrm{~T}_{3}$ and thus the Taub solution. ${ }^{17}$

The considerations of this section simplify the analysis of the silent Kasner subset $\mathcal{K}$, see Section 6.1. Since a solution of $\mathcal{K}$ is a representation of a Kasner solution in an Iwasawa frame, properties such as $u=$ const easily follow.

\section{Multiple transitions}

Here we give the proofs of some statements made in Section 6 concerning multiple transitions; as an alternative to using frame invariants, we will use elementary methods from the theory of dynamical systems.

Multiple frame transitions are solutions of (5.6) on the silent Kasner subset $\mathcal{K}$, for which at least two of the variables $\left(R_{1}, R_{2}, R_{3}\right)$ do not vanish

\footnotetext{
${ }^{17}$ BKL define the Kasner parameter $u$ according to $p_{1}=-u /\left(1+u+u^{2}\right), p_{2}=(1+$ $u) /\left(1+u+u^{2}\right), p_{3}=u(1+u) /\left(1+u+u^{2}\right)$, cf. (C.2); here, the order of the Kasner exponents is fixed as $p_{1} \leq p_{2} \leq p_{3}$ by definition. We find it more natural to use the frameindependent definition (C.1), and permute the ordering of $p_{\alpha}$ according to the sector one considers when dealing with frame-dependent matters.
} 
identically. The equations are

$$
\begin{aligned}
& \partial_{\tau} \Sigma_{1}=-2 R_{2}^{2}-2 R_{3}^{2}, \quad \partial_{\tau} \Sigma_{2}=-2 R_{1}^{2}+2 R_{3}^{2}, \quad \partial_{\tau} \Sigma_{3}=2 R_{1}^{2}+2 R_{2}^{2}, \\
& \partial_{\tau} R_{1}=\left(\Sigma_{2}-\Sigma_{3}\right) R_{1}+2 R_{2} R_{3}, \quad \partial_{\tau} R_{2}=\left(\Sigma_{1}-\Sigma_{3}\right) R_{2} \\
& \partial_{\tau} R_{3}=\left(\Sigma_{1}-\Sigma_{2}\right) R_{3}-2 R_{1} R_{2},
\end{aligned}
$$

together with $\Sigma^{2}=1$. The set $R_{2}=0$ is an invariant subset of (D.1) (while $R_{1}=0$ and $R_{3}=0$ are not invariant), hence possible classes of multiple frame transitions are $\mathcal{T}_{R_{3} R_{1}}$ (which satisfy $R_{1} \not \equiv 0, R_{2} \equiv 0, R_{3} \not \equiv 0$ ) and $\mathcal{T}_{R_{1} R_{2} R_{3}}$ (where all three functions are different from zero).

First, let $R_{2}=0$, i.e., we consider $\mathcal{T}_{R_{3} R_{1}}$ transitions. In this case, $R_{1}=0$ and $R_{3}=0$ are invariant subspaces; we consider, without loss of generality, the state space defined by $R_{1}>0$ and $R_{3}>0$; since $\Sigma^{2}=1$, the closure of this state space is compact. The functions $\Sigma_{1}$ and $\Sigma_{3}$ are monotone on the state space, and hence the monotonicity principle ${ }^{18}$ implies that the $\alpha$-limit and the $\omega$-limit of each orbit must lie on the boundaries $R_{1}=0$ (i.e., on $\overline{\mathcal{B}}_{R_{3}}$ ) or $R_{3}=0$ (i.e., on $\overline{\mathcal{B}}_{R_{1}}$ ) of the state space. Using the known structure of the flow on the boundaries (which contain the single transition orbits), we conclude that the $\alpha$-/ $\omega$-limit must be a source/sink on $\mathrm{K}^{\bigcirc}$. The fixed points in four of the sectors of $\mathrm{K}^{\bigcirc}$ are saddles; the fixed points in sector (321) are sources, since both $R_{1}$ and $R_{3}$ belong to the unstable subspaces, see figure 1 (a); the points in sector (123) are sinks. Thus, $\mathcal{T}_{R_{3} R_{1}}$ orbits originate in sector (321) and end in sector (123), see figure 4(a). (Recall that, in this paper, "time" is directed toward the past singularity; the nomenclature " $\alpha$-limit set" and " $\omega$-limit set" is used in accordance with the chosen timedirection.)

Second, let $R_{2} \neq 0$, i.e., we consider $\mathcal{T}_{R_{1} R_{2} R_{3}}$ transitions; without loss of generality we assume $R_{2}>0$. Since $\Sigma_{1}$ and $\Sigma_{3}$ are monotone functions, the monotonicity principle yields that the $\alpha$ - $/ \omega$-limit of every orbit must lie on the boundary $R_{2}=0$. Applying the previous result on the limit sets of $\mathcal{T}_{R_{3} R_{1}}$ transitions, we see that the $\alpha$-limit must be an equilibrium point in sector (321) and the $\omega$-limit a point in sector (123).

\footnotetext{
${ }^{18}$ The monotonicity principle essentially states that if there exists a function that is monotone along the orbits of a state space with compact closure, then the $\alpha$-/ $\omega$-limit set of every orbit is contained on the boundary of the state space; see $[12,49]$ and references therein.
} 
For mixed frame/curvature transitions $\mathcal{T}_{N_{1} R_{1}}$, the system (5.6) reduces to

$$
\begin{aligned}
& \partial_{\tau} \Sigma_{1}=2\left(1-\Sigma^{2}\right) \Sigma_{1}+\frac{2}{3} N_{1}^{2}, \quad \partial_{\tau} N_{1}=-2\left(\Sigma^{2}+\Sigma_{1}\right) N_{1}, \\
& \partial_{\tau} R_{1}=2\left(1-\Sigma^{2}\right) R_{1}+\left(\Sigma_{2}-\Sigma_{3}\right) R_{1}, \\
& \partial_{\tau} \Sigma_{2}=2\left(1-\Sigma^{2}\right) \Sigma_{2}-2 R_{1}^{2}-\frac{1}{3} N_{1}^{2}, \quad \partial_{\tau} \Sigma_{3}=2\left(1-\Sigma^{2}\right) \Sigma_{3}+2 R_{1}^{2}+\frac{1}{3} N_{1}^{2},
\end{aligned}
$$

together with $\left(1-\Sigma^{2}\right)=(1 / 12) N_{1}^{2}$; the remaining variables vanish identically. Without loss of generality, we consider the case $N_{1}>0$ and $R_{1}>0$. By (D.2), the function $\left(\Sigma_{1}+4\right)$ is monotone on the (relatively compact) state space, and hence the monotonicity principle guarantees that the $\alpha$ and the $\omega$-limit of every orbit resides on the boundaries $N_{1}=0$ or $R_{1}=0$. Using the structure of the flow on the boundaries (as given by the single curvature transitions of figure 3), we find that the $\alpha$-limit of each orbit is a fixed point on sector (132), and that the $\omega$-limit is a point on sector (213) or $(231)$ of $\mathrm{K}^{\circ}$, see figure $4(\mathrm{~b})$.

From (D.2) we can derive the validity of the Kasner map (6.4) explicitly. The equations (D.2a) form a two-dimensional decoupled system for $\Sigma_{1}$ and $N_{1}$ by means of the Gauss constraint; the underlying reason for this is that $\Sigma_{1}$ and $N_{1}$ are invariant under the frame rotations in the $\left\langle e_{2}, e_{3}\right\rangle$-plane that are induced by $R_{1}$. Since the subsystem for $\Sigma_{1}$ and $N_{1}$ is identical to the one for the single curvature transition case $\mathcal{T}_{N_{1}}$, the respective solutions are identical; in particular, as in (6.3a),

$$
\Sigma_{1}=-4+\left(1+u^{2}\right) \zeta
$$

where $\zeta$ is defined as in $(6.3 \mathrm{~b})$ and $u=u_{-}$. This implies that $\left(\Sigma_{1}\right)_{+}$is identical to its counterpart in the $\mathcal{T}_{N_{1}}$ case (provided that the initial values $\left(\Sigma_{1}\right)_{-}$are the same). Note, however, that $\Sigma_{2}$ and $\Sigma_{3}$ differ from the functions given in (6.3a), since $R_{1} \neq 0$ changes the evolution of these quantities. In particular, we find that $\left(\Sigma_{2}\right)_{+}^{\mathcal{T}_{N_{1} R_{1}}}=\left(\Sigma_{3}\right)_{+}^{\mathcal{T}_{N_{1}}}$ and $\left(\Sigma_{3}\right)_{+}^{\mathcal{T}_{N_{1} R_{1}}}=\left(\Sigma_{2}\right)_{+}^{\mathcal{T}_{N_{1}}}$, which reflects a relative rotation of the axes. However, in terms of the Kasner parameter $u$, the two final states are indistinguishable, which is a consequence of the frame invariance of $u$; hence $u_{+}$is given in terms of $u_{-}$ by the Kasner map (6.4).

The equations on the silent Bianchi type $\mathrm{VI}_{0} / \mathrm{VII}_{0}$ subsets are given by

$$
\partial_{\tau} \Sigma_{\alpha}=2\left(1-\Sigma^{2}\right) \Sigma_{\alpha}+{ }^{3} \mathcal{S}_{\alpha \alpha}, \quad \partial_{\tau} N_{a}=-2\left(\Sigma^{2}+\Sigma_{a}\right) N_{a},
$$


$\alpha=1, \ldots, 3, a=1,2$; here, the quantities ${ }^{3} \mathcal{S}_{\alpha \alpha}$ are polynomials in $N_{1}$ and $N_{2}$ given by (5.6i). The Gauss constraint (5.6j) reads

$$
\Sigma_{1}^{2}+\Sigma_{2}^{2}+\Sigma_{3}^{2}+\frac{1}{2}\left(N_{1}-N_{2}\right)^{2}=6 .
$$

Since ${ }^{3} \mathcal{S}_{33}=-(1 / 3)\left(N_{1}-N_{2}\right)^{2}$, we obtain

$$
\partial_{\tau}\left(2-\Sigma_{3}\right)=\frac{1}{6}\left(N_{1}-N_{2}\right)^{2}\left(2-\Sigma_{3}\right),
$$

i.e., $\left(2-\Sigma_{3}\right)$ is a strictly monotonically increasing function unless $N_{1}=N_{2}$.

The Bianchi type $\mathrm{VI}_{0}$ subset is given by $\left(N_{1}>0\right) \wedge\left(N_{2}<0\right)$ (or, equivalently, by the reversed inequalities). Hence, the Gauss constraint (D.3) leads to a compact state space given by $\Sigma_{1}^{2}+\Sigma_{2}^{2}+\Sigma_{3}^{2}+(1 / 2) N^{2}<6$, where $N_{1}=$ $N$ and $N_{2}=N-\sqrt{12\left(1-\Sigma^{2}\right)}$. The function $\left(2-\Sigma_{3}\right)$ is strictly monotone, since $\left(N_{1}-N_{2}\right)>0$ everywhere. It thus follows from the monotonicity principle that the $\alpha$ - $/ \omega$-limits of orbits must reside on the boundaries of the state space, i.e., on $\mathrm{K}^{\bigcirc} \cup \mathcal{B}_{N_{1}} \cup \mathcal{B}_{N_{2}}$. Using the known structure of the flow on the Bianchi type II subsets, see figure 3, and the resulting local properties of the fixed points on $\mathrm{K}^{\circ}$, we find that the $\alpha$-limit of each orbit is the fixed point $\mathrm{T}_{3}$ on $\mathrm{K}^{\circ}$ and that the $\omega$-limit is a point on sector (312) or (321).

The Bianchi type $\mathrm{VII}_{0}$ subset is given by $\left(N_{1}>0\right) \wedge\left(N_{2}>0\right)$ (or, equivalently, by the reversed inequalities). In this case, the Gauss constraint does not enforce a compact state space. The derivative of the function $\left(2-\Sigma_{3}\right)$ is not positive when $N_{1}=N_{2}$, see (D.4); however, $\left.3 \partial_{\tau}^{3}\left(2-\Sigma_{3}\right)\right|_{N_{1}=N_{2}}=$ $4\left(\Sigma_{1}-\Sigma_{2}\right)^{2} N_{1}^{2}\left(2-\Sigma_{3}\right)$, and hence $\left(2-\Sigma_{3}\right)$ is a strictly monotonically increasing function except on the LRS subset $N_{1}=N_{2}$ and $\Sigma_{1}=\Sigma_{2}$, which yields that $\left(\Sigma_{1}, \Sigma_{2}, \Sigma_{3}\right)=(-1,-1,2)$ or $\left(\Sigma_{1}, \Sigma_{2}, \Sigma_{3}\right)=(1,1,-2)$. The latter is an invariant subset with $\partial_{\tau} N_{a}=-4 N_{a}$, which represents the non-flat LRS Kasner solution in a Bianchi type $\mathrm{VII}_{0}$ symmetry foliation. The set $\left(\Sigma_{1}, \Sigma_{2}, \Sigma_{3}\right)=(-1,-1,2)$, on the other hand, is a one-dimensional set of fixed points, parameterized by $N_{1}=N_{2}=$ const $>0$, which we denote by $\mathcal{L}_{3}^{+}$; each fixed point represents the Minkowski spacetime in a Bianchi type $\mathrm{VII}_{0}$ symmetry foliation, see [12, p. 130, 133]. It can be proved, see [50, Theorem 5], that the $\alpha$-limit of every orbit in the Bianchi type $\mathrm{VII}_{0}$ state space is one of the fixed points on $\mathcal{L}_{3}^{+}$. The $\omega$-limits are points on sectors $(312)$ or (321) of $\mathrm{K}^{\bigcirc}$.

For each individual Bianchi type $\mathrm{VI}_{0}$ or $\mathrm{VII}_{0}$ orbit, the quantity $\left|N_{1}\right|$ (and, equivalently, $\left|N_{2}\right|$ ) goes through a maximum value. It is of interest to note that there exists a uniform bound, i.e., there exists $\epsilon>0$ such 
that $\max _{\tau}\left|N_{1}(\tau)\right| \geq \epsilon$ (and $\max _{\tau}\left|N_{2}(\tau)\right| \geq \epsilon$ ) uniformly for all Bianchi type $\mathrm{VI}_{0} / \mathrm{VII}_{0}$ orbits. In order to see this, assume that the assertion is false; then there exists for all $n \in \mathbb{N}$ a Bianchi type $\mathrm{VI}_{0}$ (Bianchi type $\mathrm{VII}_{0}$ ) orbit $\mathfrak{T}_{n}$ such that $\max _{\tau}\left|N_{1}(\tau)\right|<1 / n$, i.e., $\left|N_{1}(\tau)\right|<1 / n \forall \tau$. In particular, $\left|N_{1}\left(\tau_{n}\right)\right|<1 / n$, where $\tau_{n}$ is such that $\Sigma_{3}\left(\tau_{n}\right)=-1$. Since the state space is compact (or, in Bianchi type $\mathrm{VII}_{0}$, the intersection of the state space with the set $\left|N_{1}\right| \leq 1$ ), without loss of generality the $\operatorname{limit}_{n \rightarrow \infty} \Sigma_{\alpha}\left(\tau_{n}\right)$ exists - otherwise we go over to a subsequence. If the limit is neither of the points $\mathrm{Q}_{1}, \mathrm{Q}_{2}$, then $\left(\Sigma_{\alpha}, N_{1}, N_{2}\right)\left(\tau_{n}\right)$ converges to a point on a $\mathcal{T}_{N_{2}}$ single curvature transition orbit. Hence, for sufficiently large $n$, the corresponding orbit $\mathfrak{T}_{n}$ shadows this $\mathcal{T}_{N_{2}}$ transition. However, the flow on the boundary of $\mathcal{B}_{N_{1} N_{2}-}$ $\left(\mathcal{B}_{N_{1} N_{2}+}\right)$ forces $\mathfrak{T}_{n}$ to also shadow the $\mathcal{T}_{N_{1}}$ transition preceding/succeeding it. Along these $\mathcal{T}_{N_{1}}$ transitions we have $\left|N_{1}\right| \nless(1 / n)$ for large $n$, which is a contradiction. The argument is analogous when $\lim _{n \rightarrow \infty} \Sigma_{\alpha}\left(\tau_{n}\right)$ is one of the points $\mathrm{Q}_{1}, \mathrm{Q}_{2}$; in this case we employ the saddle structure of these fixed points to show that for sufficiently large $n$, the orbit $\mathfrak{T}_{n}$ shadows a $\mathcal{T}_{N_{1}}$ transition along which $\left|N_{1}\right| \nless(1 / n)$, which is again the desired contradiction.

\section{E Behavior of an auxiliary quantity}

In this appendix, we consider equation (9.6), i.e.,

$$
\frac{\partial}{\partial \tau} B=2\left(1-\Sigma^{2}\right) B+2 R_{3}^{2}-2 R_{1}^{2}
$$

we show that, generically, $B_{i} \sim \operatorname{const} G_{l(i)}$ as $i \rightarrow \infty$, cf. (9.8), along a sequence of transitions $\mathcal{S}_{\mathcal{T}}$. (In Section 9, this statement was shown to be true for sequences $\mathcal{S}_{\mathcal{T}}$ that do not contain $\mathcal{T}_{N_{2}}$ and $\mathcal{T}_{N_{2} R_{2}}$ transitions, which is equivalent to assuming $N_{2} \equiv 0$.)

For the following it is convenient to use a continuous time variable along $\mathcal{S}_{\mathcal{T}}$; we introduce "sequence time" $\lambda$ by $\lambda=(1 / 2)+(\arctan \tau) / \pi$, i.e., $\tau=$ $\tan [(2 \lambda-1) \pi / 2]$; accordingly, the time interval $\lambda \in(i, i+1)($ for $i \in \mathbb{N})$ corresponds to $\tau$ ranging in $(-\infty, \infty)$ during the $i$ th transition. By using sequence time, we define the growth function $G$ by

$$
G(\lambda): \quad \partial_{\lambda} G=2\left(1-\Sigma^{2}\right) \frac{d \tau}{d \lambda} G, \quad G(0)=1
$$

By construction, $G(\lambda)$ coincides with $G_{l(i)}$ as defined in Section 9 for $\lambda=i$; this is because $G_{l}=\prod_{k=0}^{l-1} g_{k}$, where the growth factors $g_{k}$ are defined 
as the ratio $\zeta_{+} / \zeta_{-}$(where $\zeta_{ \pm}=\lim _{\tau \rightarrow \pm \infty} \zeta$ ) that arises from the equation $\partial_{\tau} \zeta=2\left(1-\Sigma^{2}\right) \zeta$ for the $k$ th curvature transition, see (6.5) and Section 9.

The derivative of $B$ is closely related to derivatives of $\Sigma_{2}$; in sequence time $\lambda$ we find

$$
\frac{\partial}{\partial \lambda} B= \begin{cases}\frac{\partial}{\partial \lambda}\left(\Sigma_{2}-2\right)+2\left(1-\Sigma^{2}\right) \frac{d \tau}{d \lambda}\left[B-\left(\Sigma_{2}-2\right)\right] & \text { when } N_{2}=0 \\ \frac{\partial}{\partial \lambda}\left(\Sigma_{2}+4\right)+2\left(1-\Sigma^{2}\right) \frac{d \tau}{d \lambda}\left[B-\left(\Sigma_{2}+4\right)\right] & \text { when } N_{1}=0\end{cases}
$$

Integrating (E.1) thus amounts to integrating the two equations in (E.2) alternately: as long as $N_{2}=0$ we use the first equation; whenever there is a $\mathcal{T}_{N_{2}}$ or a $\mathcal{T}_{N_{2} R_{2}}$ transition the second equation is employed. For $k \in \mathbb{N}$, let $\lambda_{k} \in \mathbb{N}$ be a sequence of times that represents the "switches" between the two equations in (E.2), i.e., $N_{2} \equiv 0$ in $\left(\lambda_{k}, \lambda_{k+1}\right)$ for even $k$ and $N_{1} \equiv 0$ in $\left(\lambda_{k}, \lambda_{k+1}\right)$ for odd $k$. Integration of (E.2) and a somewhat cumbersome iteration yields

$B(\lambda)=\left(\Sigma_{2}-2\right)(\lambda)+6 G(\lambda)\left[\sum_{j=1}^{k}(-1)^{j} \frac{1}{G\left(\lambda_{j}\right)}\right]+G(\lambda)\left[B(0)-\left(\Sigma_{2}-2\right)(0)\right]$

for $\lambda \in\left(\lambda_{k}, \lambda_{k+1}\right)$ with $k$ even (and a similar result for odd $k$ ). By the Leibniz criterion, the sum $\sum_{j=1}^{k}(-1)^{j} G\left(\lambda_{j}\right)^{-1}$ converges as $k \rightarrow \infty$. Moreover, $\left|\sum_{j>k}(-1)^{j} G\left(\lambda_{j}\right)^{-1}\right|<G\left(\lambda_{j+1}\right)^{-1}$, hence $G(\lambda) \sum_{j>k}(-1)^{j} G\left(\lambda_{j}\right)^{-1}$ is bounded. Since also $\left(\Sigma_{2}-2\right)(\lambda)$ is bounded, we can replace the sum with an infinite series and collect the remaining terms in a term labeled $b(\lambda)$,

$$
B(\lambda)=G(\lambda)\left[B(0)+6 \sum_{j=1}^{\infty}(-1)^{j} \frac{1}{G\left(\lambda_{j}\right)}-\left(\Sigma_{2}-2\right)(0)\right]+b(\lambda)
$$

here $b(\lambda)$ is bounded (and oscillatory). Consequently, generically, $B(\lambda) \sim$ const $G(\lambda)$ holds asymptotically, or, in different notation, $B_{i} \sim$ const $G_{l(i)}$.

\section{F Convergence of sums}

Consider a sequence of transitions $\mathcal{S}_{\mathcal{T}}$ on the oscillatory subset $\mathcal{O}$; in our nomenclature, $\mathcal{S}_{\mathcal{T}}$ denotes a generic sequence, i.e., a sequence that does not contain any double curvature transitions, see Section 7; therefore, the 
sequence $\mathcal{S}_{\mathcal{T}}$ is associated with a Kasner sequence $\left(u_{l}\right)_{l \in \mathbb{N}}$, where $l \in \mathbb{N}$ is the index that consecutively numbers the curvature transitions of $\mathcal{S}_{\mathcal{T}}$.

In this appendix we first analyze the series

$$
\sum_{l} G_{l}^{-1}\left|\left(\Delta \Sigma_{2}\right)\right|_{l}
$$

where $G_{l}$ is the growth function and $\left|\left(\Delta \Sigma_{2}\right)\right|_{l}$ denotes the sum of the absolute changes in $\Sigma_{2}$ occurring for the frame transitions between the $(l-1)$ th and the $l$ th curvature transition. Series of the type (F.1) arise in connection with the asymptotic suppression of the variable $N_{2}$, see Section 10 and the discussion below, and in the context of the asymptotic freezing of the Hamiltonian variables $n_{i}$ and $\mathcal{P}_{i}$, see Appendix $\mathrm{H}$. In the following, we prove that the series (F.1) converges. To establish this result, we analyze contributions of the sum in large and small curvature phases separately.

First, we consider (F.1) for a large curvature phase (which we assume to be associated with a sufficiently large value of $\left.\eta_{u}\right): l \in\left[l_{\mathrm{i}}, l_{\mathrm{f}}\right]$. Obviously, $\left|\left(\Delta \Sigma_{2}\right)\right|_{l}$ is uniformly bounded (by $\left.2 \sqrt{3}\right)$, so that $G_{l}^{-1}\left|\left(\Delta \Sigma_{2}\right)\right|_{l} \leq$ const $G_{l}^{-1}$. Furthermore, in a large curvature phase, $g_{l}>C_{\mathrm{p}}>1$, cf. (9.11), so that $G_{l+1}^{-1}=g_{l}^{-1} G_{l}^{-1}<G_{l}^{-1} C_{\mathrm{p}}^{-1}$ for all $l \in\left[l_{\mathrm{i}}, l_{\mathrm{f}}\right]$. Hence, for a large curvature phase, each term in the sum can be bounded by a term of a geometric series.

Second, we consider (F.1) for a small curvature phase; since small curvature phases dominate over large curvature phases in the probabilistic description of sequences, see Section 8 , the subsequent considerations are of central significance. Recall that the prototype of a small curvature phase is the alternating sequence of $\mathcal{T}_{N_{1}}$ and $\mathcal{T}_{R_{3}}$ transitions in a neighborhood of the point $\mathrm{T}_{3}$, which is characteristic for billiard sequences $\mathcal{B}_{\mathcal{T}}$. The following considerations are adapted to small curvature phases of this kind; however, the results hold for general small curvature phases with obvious minor modifications. Let $l \in\left[l_{\text {in }}, l_{\text {out }}\right]$ be a small curvature phase (where we assume $u^{\text {in }}>\eta_{u}+1$ to obtain a phase consisting of at least two-curvature transitions):

$$
\begin{aligned}
\sum_{l=l_{\text {in }}+1}^{l_{\text {out }}+1} G_{l}^{-1}\left|\left(\Delta \Sigma_{2}\right)\right|_{l} & =\sum_{l=l_{\text {in }}+1}^{l_{\text {out }}+1} G_{l_{\text {in }}}^{-1}\left[\prod_{j=l_{\text {in }}}^{l-1} g_{j}\right]^{-1}\left|\left(\Delta \Sigma_{2}\right)\right|_{l} \\
& =G_{l_{\text {in }}}^{-1} \sum_{l=l_{\text {in }}+1}^{l_{\text {out }}+1}\left(\frac{1+u_{l}+u_{l}^{2}}{1+u^{\text {in }}+\left(u^{\text {in }}\right)^{2}}\right)\left(\frac{3\left(1+2 u_{l}\right)}{1+u_{l}+u_{l}^{2}}\right)
\end{aligned}
$$




$$
\begin{aligned}
& =G_{l_{\mathrm{in}}}^{-1} \sum_{k=1}^{L} \frac{3\left(1+2\left(u^{\mathrm{in}}-k\right)\right)}{1+u^{\mathrm{in}}+\left(u^{\mathrm{in}}\right)^{2}}=G_{l_{\mathrm{in}}}^{-1} \frac{3 L\left(2 u^{\mathrm{in}}-L\right)}{1+u^{\mathrm{in}}+\left(u^{\mathrm{in}}\right)^{2}} \\
& \approx G_{l_{\mathrm{in}}}^{-1} \frac{3\left(\left(u^{\mathrm{in}}\right)^{2}-\eta_{u}^{2}\right)}{1+u^{\mathrm{in}}+\left(u^{\mathrm{in}}\right)^{2}}<3 G_{l_{\mathrm{in}}}^{-1}<\operatorname{const} G_{l_{\mathrm{in}}}^{-1},
\end{aligned}
$$

where we have used (9.9) for the growth factors $g_{j}$ and (6.2) for $\left|\left(\Delta \Sigma_{2}\right)\right|_{l}$.

From $(9.12)$ we see that $G_{l_{\text {in }}}^{-1}<G_{l_{\text {in }}-1}^{-1} C_{\mathrm{p}}^{-1}$; furthermore, $G_{l_{\text {out }}+2}^{-1}<G_{l_{\text {in }}}^{-1}$ $\left(1-\eta_{u}+\eta_{u}^{2}\right) /\left(1+u^{\text {in }}+\left(u^{\text {in }}\right)^{2}\right)$, cf. $(9.10)$, so that $G_{l_{\text {out }}+2}^{-1}<G_{l_{\text {in }}}^{-1} C_{\mathrm{p}}^{-1}$. This leads to the important conclusion that the sum associated with a small curvature phase can be treated on an equal footing with a single term in a large curvature phase, i.e.,

$$
\begin{aligned}
\sum_{l} G_{l}^{-1}\left|\left(\Delta \Sigma_{2}\right)\right|_{l}= & \cdots+\left.\left(\Delta \Sigma_{2}\right)\right|_{l_{\text {in }}-1} G_{l_{\text {in }}-1}^{-1}+\left.\left(\Delta \Sigma_{2}\right)\right|_{l_{\text {in }}} G_{l_{\text {in }}}^{-1} \\
& +\left[\sum_{l=l_{\text {in }}+1}^{l_{\text {out }}+1} G_{l}^{-1}\left|\left(\Delta \Sigma_{2}\right)\right|_{l}\right]+\left.\left(\Delta \Sigma_{2}\right)\right|_{l_{\mathrm{out}}+2} G_{l_{\text {out }}+2}^{-1}+\cdots \\
& <\operatorname{const}\left(\cdots+G_{l_{\text {in }}-1}^{-1}+G_{l_{\text {in }}}^{-1}+G_{l_{\text {in }}}^{-1}+G_{l_{\text {out }}+2}^{-1}+\ldots\right) \\
& <\operatorname{const}\left(\cdots+G_{l_{\text {in }}-1}^{-1}+G_{l_{\text {in }}-1}^{-1} C_{\mathrm{p}}^{-1}+G_{l_{\text {in }}-1}^{-1} C_{\mathrm{p}}^{-1}\right. \\
& \left.+G_{l_{\text {in }}-1}^{-1} C_{\mathrm{p}}^{-2}+\cdots\right)
\end{aligned}
$$

Therefore,

$$
\sum_{l} G_{l}^{-1}\left|\left(\Delta \Sigma_{2}\right)\right|_{l} \leq \mathrm{const} \sum_{k} C_{\mathrm{p}}^{-k}<\infty,
$$

which proves the asserted convergence of the series.

For asymptotic sequences of transitions $\mathcal{A S}_{\mathcal{T}}$ the above considerations apply in the asymptotic regime when $\mathcal{A S}_{\mathcal{T}}$ shadows sequences on $\mathcal{O}$ and thus consists of approximate transitions $\left(\mathcal{T}_{i}\right)_{i \in \mathbb{N}}$. The convergence result also holds analogously for asymptotic sequences $\mathcal{A S}_{\mathcal{O}}$ : since the possible (approximate) Bianchi type $\mathrm{VI}_{0}$ and $\mathrm{VII}_{0}$ orbits are associated with a very large increase in $G$, convergence in the sum is strengthened.

The established convergence of (F.1) has direct applications in our discussion of asymptotic freezing in Appendix H. The convergence result is also the basis for the proof of the assertion made in Section 10 that equation (10.4) 
simplifies to (10.5) along an asymptotic sequence of transitions $\mathcal{A S}_{\mathcal{T}}$; this will been shown in the following:

Integration of (10.4), i.e.,

$$
\partial_{\tau}\left(N_{1} R_{3}^{2}\right)=2\left[2\left(1-\Sigma^{2}\right)-\Sigma^{2}-\Sigma_{2}\right] N_{1} R_{3}^{2}-4 R_{1}^{2} G^{-1}\left(N_{1} R_{3}^{2}\right),
$$

yields

$$
\log \left(N_{1} R_{3}^{2}\right)=2 \int\left(2\left(1-\Sigma^{2}\right)-\Sigma^{2}-\Sigma_{2}\right) d \tau-4 \int R_{1}^{2} G^{-1} d \tau+\text { const. }
$$

In the asymptotic regime, the integrals can be written as sums over the (approximate) transitions of the asymptotic sequence; the second integral contributes only for transitions involving $R_{1}$. We can make the estimate

$$
\int R_{1}^{2} G^{-1} d \tau \leq \sum_{l} G_{l+1}^{-1} \int_{\tau_{l}}^{\tau_{l+1}} R_{1}^{2} d \tau,
$$

where the limits of the integral on the r.h.s. denote the $\tau$-time that elapses during the frame transitions between curvature transition number $l$ and $l+1$. In the asymptotic regime, the integrals of this type can be approximated by using exact transitions; in particular, there exists a uniform upper bound, which suffices to prove convergence in large curvature phases in complete analogy to the considerations above.

The variable $R_{1}$ is non-zero along $\mathcal{T}_{R_{1}}$ transitions; along other single transitions it vanishes. Note that multiple transitions involving $R_{1}$ can be ignored in the present context, since we focus on small curvature phases, where multiple transitions do not appear. For a $\mathcal{T}_{R_{1}}$ transitions we have $-2 R_{1}^{2}=\partial_{\tau} \Sigma_{2}$, hence $-2 \int_{\tau_{l}}^{\tau_{l+1}} R_{1}^{2} d \tau=\left(\Sigma_{2}\right)_{+}-\left(\Sigma_{2}\right)_{-}$. Therefore, since $\left|2 \int_{\tau_{l}}^{\tau_{l+1}} R_{1}^{2}\right| \leq|\Delta \Sigma|_{l}$, the sum in (F.4) can be estimated by a sum of the type (F.1); we thus obtain convergence and

$$
\log \left(N_{1} R_{3}^{2}\right)=2 \int\left(2\left(1-\Sigma^{2}\right)-\Sigma^{2}-\Sigma_{2}\right) d \tau+\text { const }^{\prime}
$$

as $\tau \rightarrow \infty$. Consequently, the term $-4 R_{1}^{2} G^{-1}\left(N_{1} R_{3}^{2}\right)$ in (F.3) can be neglected as $\tau \rightarrow \infty$; this leads to the simplified equation (10.5) and thus establishes the claim.

To prove asymptotic freezing of $\pi_{\lambda}$ in Appendix $\mathrm{H}$, we need to consider series of a slightly more general type than (F.1). Consider a sequence of 
transitions $\mathcal{S}_{\mathcal{T}}$ and the associated series

$$
\sum_{l} G_{l}^{-a} u_{l}^{-c}
$$

where $a>0, c>0$ (and for simplicity $2 a-c>-1$ ); note in this context that the series

$$
\sum_{l} G_{l}^{-1} \log G_{l} \frac{1}{u_{l}}, \quad \sum_{l} G_{l}^{-1} \frac{\log u_{l}}{u_{l}}
$$

see (H.20), can be estimated by a series of the type (F.5) (with $a=1-\epsilon$, $c=1-\epsilon)$. In the following we prove convergence of the series (F.5).

In analogy to (F.2), we obtain during a small curvature phase

$$
\begin{aligned}
\sum_{l=l_{\text {in }}+1}^{l_{\text {out }}+1} G_{l}^{-a}\left(u_{l}\right)^{-c} & =G_{l_{\text {in }}}^{-a} \sum_{l=l_{\text {in }}+1}^{l_{\text {out }}+1}\left(\frac{1+u_{l}+u_{l}^{2}}{1+u^{\text {in }}+\left(u^{\text {in }}\right)^{2}}\right)^{a}\left(u_{l}\right)^{-c} \\
& <(1+\epsilon) G_{l_{\text {in }}}^{-a} \sum_{l=l_{\text {in }}+1}^{l_{\text {out }}+1} \frac{\left(u_{l}\right)^{2 a-c}}{\left(u^{\text {in }}\right)^{2 a}}
\end{aligned}
$$

where we have used an estimate of the type $\left(1+u+u^{2}\right)<(1+\epsilon) u^{2}$ for some $\epsilon>0$ (which is small when $\eta_{u}$ is large). Further, by estimating the sum through the associated integral, i.e.,

$$
\sum_{l=l_{\text {in }}+1}^{l_{\text {out }}+1}\left(u_{l}\right)^{2 a-c}=\sum_{k=1}^{L}\left(u^{\text {in }}-k\right)^{2 a-c} \leq \mathrm{const} \int_{\eta_{u}}^{u^{\mathrm{in}}} u^{2 a-c} d u
$$

we arrive at

$$
\sum_{l=l_{\text {in }}+1}^{l_{\text {out }}+1} G_{l}^{-a}\left(u_{l}\right)^{-c}<\operatorname{const} G_{l_{\text {in }}}^{-a} \frac{1}{2 a-c+1}\left(u^{\text {in }}\right)^{1-c}=\operatorname{const} G_{l_{\text {in }}}^{-a}\left(u^{\text {in }}\right)^{1-c}
$$

Modulo the large curvature phase terms whose sum converges straightforwardly, and which hence can be suppressed, the series (F.5) can be written as

$$
\sum_{l} G_{l}^{-a}\left(u_{l}\right)^{-c}=\sum_{j} \sum_{l=l_{\mathrm{in}, j}+1}^{l_{\mathrm{out}, j}+1} G_{l}^{-a}\left(u_{l}\right)^{-c}
$$


where $\left[\left(l_{\text {in }}\right)_{j},\left(l_{\text {out }}\right)_{j}\right]$ denotes the $j$ th small curvature phase. Based on the estimate (F.6) we therefore obtain

$$
\sum_{l} G_{l}^{-a}\left(u_{l}\right)^{-c}<\text { const } \sum_{j}\left(G_{\left(l_{\mathrm{in}}\right)_{j}}\right)^{-a}\left(u_{j}^{\mathrm{in}}\right)^{1-c},
$$

where $u_{j}^{\text {in }}$ is the initial value of $u$ for the small curvature phase number $j$, which begins with transition $l_{\text {in }, j}$.

Regarded as a random variable, $u^{\text {in }}=\varkappa^{-1}$ is associated with the probability density $\hat{w}(\varkappa)$, see (8.5). Therefore, $u^{\text {in }}=\varkappa^{-1}$ does not possess a finite expectation value, cf. Section 8 ; however, $\left(u^{\text {in }}\right)^{1-c}=\varkappa^{c-1}$ does, since

$$
\left\langle\left(u^{\mathrm{in}}\right)^{1-c}\right\rangle=\int_{0}^{\eta_{u}^{-1}} \hat{w}(\varkappa) \varkappa^{c-1} d \varkappa \sim \hat{w}(0) \frac{1}{c}\left(\eta_{u}^{-1}\right)^{c} \sim \frac{1}{c} \eta_{u}^{1-c} .
$$

It follows that the expectation value of the sum in (F.7) exists, i.e.,

$$
\left\langle\sum_{j=1}^{n}\left(G_{\left(l_{\mathrm{in}}\right)_{j}}\right)^{-a}\left(u_{j}^{\mathrm{in}}\right)^{1-c}\right\rangle=\sum_{j=1}^{n}\left(G_{\left(l_{\mathrm{in}}\right)_{j}}\right)^{-a} \frac{\eta_{u}^{1-c}}{c} .
$$

Hence, since $G_{\left(l_{\text {in }}\right)_{j}}$ increases geometrically, i.e., $G_{\left(l_{\text {in }}\right)_{j+1}}>C_{\mathrm{p}} G_{\left(l_{\text {in }}\right)_{j}}$, the limit $n \rightarrow \infty$ exists. We conclude that the series (F.7) and thus (F.5) converges.

\section{G Hitting intervals stochastically}

Let us introduce a continuous random variable $\varkappa \in[0,1]$ with probability density

$$
w:[0,1] \ni \varkappa \mapsto w(\varkappa) \in \mathbb{R}, \quad w(0)>0 .
$$

A simple example is a uniformly distributed random variable, i.e., $w(\varkappa) \equiv 1$.

Now consider a sequence $\left(\delta_{n}\right)_{n \in \mathbb{N}}$ where $\delta_{n} \in(0,1) \forall n$ and where $\delta_{n} \rightarrow 0$ $(n \rightarrow \infty)$. The sequence $\left(\delta_{n}\right)_{n \in \mathbb{N}}$ generates a sequence of intervals $\left[0, \delta_{n}\right]$ whose length decreases as $n \rightarrow \infty$. 
The probability that $\varkappa \in\left[0, \delta_{n}\right]$ is given by

$$
P\left(\varkappa \in\left[0, \delta_{n}\right]\right)=\int_{0}^{\delta_{n}} w(\varkappa) d \varkappa=: \tilde{\delta}_{n} \approx w(0) \delta_{n},
$$

where the approximation holds for $\delta_{n} \ll 1$. (Evidently, the result is exact for a uniformly distributed random variable.) We denote the event $\varkappa \in$ $\left[0, \delta_{n}\right]$ as a "hit"; the associated probability $P\left(\varkappa \in\left[0, \delta_{n}\right]\right)=\tilde{\delta}_{n}$ is the "hit probability".

Consider a series of $n=1, \ldots, N$ trials. The probability that no hits occur during $N$ trials, i.e., $\varkappa \notin\left[0, \delta_{n}\right] \forall n=1, \ldots, N$ ("no hits"), is given by

$$
P^{N}(0)=\prod_{n=1}^{N}\left(1-\tilde{\delta}_{n}\right)
$$

analogously, the probability for "one hit" is

$$
\begin{aligned}
P^{N}(1)=\sum_{n=1}^{N} \tilde{\delta}_{n} \prod_{j=1, j \neq n}^{N}\left(1-\tilde{\delta}_{j}\right) & =\left(\prod_{n=1}^{N}\left(1-\tilde{\delta}_{n}\right)\right) \sum_{i=1}^{N} \frac{\tilde{\delta}_{i}}{1-\tilde{\delta}_{i}} \\
& =P^{N}(0) \sum_{i=1}^{N} \frac{\tilde{\delta}_{i}}{1-\tilde{\delta}_{i}} .
\end{aligned}
$$

Finally, the probability for " $N$ hits" is the product $\prod_{n=1}^{N} \tilde{\delta}_{n}$.

As a simple example let $\delta_{n}=\epsilon n^{-1}$ with $\epsilon \ll 1$. We obtain

$$
\begin{aligned}
\log P^{N}(0) & =\sum_{n=1}^{N} \log \left(1-\tilde{\delta}_{n}\right) \approx \sum_{n=1}^{N}\left(-w(0) \delta_{n}+O\left(\delta_{n}^{2}\right)\right) \\
& =-\epsilon w(0) \sum_{n=1}^{N} \frac{1}{n}+O\left(\epsilon^{2}\right)
\end{aligned}
$$

therefore $\log P^{N}(0) \rightarrow-\infty$ as $N \rightarrow \infty$, and $P^{N}(0) \rightarrow 0$ as $N \rightarrow \infty$ (independently of $\epsilon$ ). Similarly,

$$
P^{N}(1)=P^{N}(0) \sum_{i=1}^{N} \frac{\tilde{\delta}_{i}}{1-\tilde{\delta}_{i}} \approx \exp \left(-\epsilon w(0) \sum_{n=1}^{N} \frac{1}{n}\right)\left[\epsilon w(0) \sum_{n=1}^{N} \frac{1}{n}\right] ;
$$

therefore $P^{N}(1) \rightarrow 0$ as $N \rightarrow \infty$ (independently of $\epsilon$ ). The analogous result holds for the general case: one can show that $P^{N}(i) \rightarrow 0$ as $N \rightarrow \infty$ for all 
$i \in \mathbb{N}$. We conclude that for an infinite series of trials the probability of getting a finite number $i$ of hits is zero, $P^{\infty}(i)=0$. We expect an infinite number of hits as $N \rightarrow \infty$ (although the intervals between two hits are expected to become increasingly large).

As a second simple example let $\delta_{n}=\epsilon n^{-2}$ with $\epsilon \ll 1$. We obtain

$$
\begin{aligned}
\log P^{N}(0) & =\sum_{n=1}^{N} \log \left(1-\tilde{\delta}_{n}\right) \approx \sum_{n=1}^{N}\left(-w(0) \delta_{n}+O\left(\delta_{n}^{2}\right)\right) \\
& =-\epsilon w(0) \sum_{n=1}^{N} \frac{1}{n^{2}}+O\left(\epsilon^{2}\right)
\end{aligned}
$$

in this case, $\log P^{N}(0) \rightarrow-c$ as $N \rightarrow \infty$ (with $\left.c>0\right)$, hence $P^{N}(0) \rightarrow e^{-c}>0$ as $N \rightarrow \infty$. Analogously, one can show that $P^{N}(i) \rightarrow$ const $>0$ as $N \rightarrow \infty$ for all $i \in \mathbb{N}$; in addition, $P^{N}(i) \leq \mathrm{P}^{N}(0)\left(c^{i} / i\right.$ ! $)$. Accordingly, for an infinite series of trials, the probability of getting a finite number $i$ of hits is finite, i.e., $P^{\infty}(i)>0$, where $P^{\infty}(i) \rightarrow 0$ as $i \rightarrow \infty$. An important conclusion is that the expectation value of the number of hits is finite,

$$
\langle \# \text { hits }\rangle=\sum_{i=1}^{\infty} i P^{\infty}(i)<\infty .
$$

Note that the expectation value exists because $P^{\infty}(i)$ falls off sufficiently rapidly as $i \rightarrow \infty$.

Remark. For the above computations we have made use of the approximation $\tilde{\delta}_{n} \approx w(0) \delta_{n}$, cf. (G.1). Alternatively, we could have used an estimate of the type $\tilde{\delta}_{n} \leq\|w\|_{\infty} \delta_{n}$.

\section{H Asymptotic constants of the motion}

The analysis of Damour et al. [13] indicates that the off-diagonal Iwasawa frame variables $n_{i}$, their conjugate momenta $\mathcal{P}_{i}$, and the momentum $\pi_{\lambda}$ conjugate to $\lambda=\log \rho$, see Section 2, are asymptotic constants of motion; i.e., these quantities converge to functions that only depend on the spatial variables (so-called asymptotic freezing). In this appendix, we will derive these results from a dynamical systems perspective. 


\section{H.1 Asymptotic freezing of $n_{i}$ and $\mathcal{P}_{i}$}

We begin by establishing asymptotic freezing of the quantities $\bar{n}_{i}$ and $\mathcal{P}_{i}$. In the conformal Hubble-normalized variables, these quantities are given by

$$
\begin{aligned}
& \bar{n}_{1}=\frac{E_{2}{ }^{1}}{E_{2}}, \quad \bar{n}_{2}=\frac{E_{3}{ }^{1}}{E_{3}}, \quad \bar{n}_{3}=\frac{E_{3}{ }^{2}}{E_{3}} \\
& \mathcal{P}_{1}=-2 H \sqrt{g} R_{3} \frac{E_{2}}{E_{1}}+\bar{n}_{3} \mathcal{P}_{2}, \quad \mathcal{P}_{2}=2 H \sqrt{g} R_{2} \frac{E_{3}}{E_{1}}, \quad \mathcal{P}_{3}=-2 H \sqrt{g} R_{1} \frac{E_{3}}{E_{2}},
\end{aligned}
$$

where we have used the notation

$$
E_{1}:=E_{1}{ }^{1}, \quad E_{2}:=E_{2}{ }^{2}, \quad E_{3}:=E_{3}{ }^{3} ;
$$

see Appendix A for details. Since $n_{1}=-\bar{n}_{1}, n_{2}=-\bar{n}_{2}+\bar{n}_{1} \bar{n}_{3}, n_{3}=-\bar{n}_{3}$, cf. (2.6), asymptotic freezing of $\bar{n}_{i}$ implies that also $n_{i}$ converge to constants.

Consider the quantities $\bar{n}_{i}$. From the formulas for $\partial_{0} E_{\alpha}{ }^{i}$, see Appendix A, it is straightforward to compute

$$
\partial_{0} \bar{n}_{1}=2 R_{3} \frac{E_{1}}{E_{2}}, \quad \partial_{0} \bar{n}_{2}=-2 R_{2} \frac{E_{1}}{E_{3}}+2 R_{1} \frac{E_{2}{ }^{1}}{E_{3}}, \quad \partial_{0} \bar{n}_{3}=2 R_{1} \frac{E_{2}}{E_{3}} .
$$

Let us now consider a sequence of transitions (or a billiard sequence) that is approximated by an attractor sequence $\mathcal{A}_{\mathcal{T}}$ in the asymptotic regime $\tau \rightarrow \infty$. Recall that $\mathcal{A}_{\mathcal{T}}$ is an infinite concatenation of $\mathcal{T}_{N_{1}}, \mathcal{T}_{R_{1}}$, and $\mathcal{T}_{R_{3}}$ transitions on the billiard attractor $\mathcal{O}_{\mathcal{B} \mathcal{A}}$, see Section 11. As usual, for notational simplicity, we drop the distinction between exact transitions and approximate transitions. To lowest order, the evolution equations for $\bar{n}_{i}$ along $\mathcal{A}_{\mathcal{T}}$ are given by

$$
\partial_{\tau} \bar{n}_{1}=-2 R_{3} \frac{E_{1}}{E_{2}}, \quad \partial_{\tau} \bar{n}_{2}=-2 \bar{n}_{1} R_{1} \frac{E_{2}}{E_{3}}, \quad \partial_{\tau} \bar{n}_{3}=-2 R_{1} \frac{E_{2}}{E_{3}} .
$$

Let us first focus on the evolution of $\bar{n}_{1}$. We rewrite the evolution equation as

$$
\partial_{\tau} \bar{n}_{1}=-2 R_{3}^{2}\left(\frac{E_{1}}{E_{2} R_{3}}\right)
$$

and investigate the behavior of the quantity $E_{1} /\left(E_{2} R_{3}\right)$. Along $\mathcal{A}_{\mathcal{T}}$ we obtain

$$
\frac{\partial}{\partial \tau}\left(\frac{E_{1}}{E_{2} R_{3}}\right)=-2\left(1-\Sigma^{2}\right)\left(\frac{E_{1}}{E_{2} R_{3}}\right) ;
$$


therefore, $E_{1} /\left(E_{2} R_{3}\right)$ behaves like the inverse of the auxiliary quantity $A$, see (9.1). Accordingly, $E_{1} /\left(E_{2} R_{3}\right)$ decreases like the inverse of the growth function, i.e.,

$$
\left(\frac{E_{1}}{E_{2} R_{3}}\right)_{i}=G_{l(i)}^{-1}\left(\frac{E_{1}}{E_{2} R_{3}}\right)_{0},
$$

see (9.5). Inserting this result into (H.3), we are able to explicitly compute the change in $\bar{n}_{1}$ for each transition: $\Delta \bar{n}_{1}$ is zero (in the lowest order approximation) for each $\mathcal{T}_{R_{1}}$ and each $\mathcal{T}_{N_{1}}$ transition and

$$
\left(\Delta \bar{n}_{1}\right)_{i}=-\left(\frac{E_{1}}{E_{2} R_{3}}\right)_{i}\left|\Delta \Sigma_{2}\right|_{i}=-\operatorname{const} G_{l(i)}^{-1}\left|\Delta \Sigma_{2}\right|_{i},
$$

when $\mathcal{T}_{i}$ is a $\mathcal{T}_{R_{3}}$ frame transition; $\left(\Delta \Sigma_{2}\right)_{i}$ denotes the change in $\Sigma_{2}$ during this transition; here we have used that $E_{1} /\left(E_{2} R_{3}\right)$ is constant during $\mathcal{T}_{i}$ and that $\partial_{\tau} \Sigma_{2}=2 R_{3}^{2}$.

To establish asymptotic freezing of $\bar{n}_{1}$, we must show that the summation of all $\left(\Delta \bar{n}_{1}\right)_{i}$ is finite (where the sum is over all $\mathcal{T}_{R_{3}}$ frame transitions of $\left.\mathcal{A}_{\mathcal{T}}\right)$. Let $\mathcal{I}_{R_{3}}=\left\{i \mid \mathcal{T}_{i}\right.$ is $\left.\mathcal{T}_{R_{3}}\right\}$; the series

$$
\sum_{i \in \mathcal{I}_{R_{3}}}\left(\Delta \bar{n}_{1}\right)_{i}=\text { const } \sum_{i \in \mathcal{I}_{R_{3}}} G_{l(i)}^{-1}\left|\Delta \Sigma_{2}\right|_{i}
$$

can be rewritten as

$$
\sum_{l} G_{l}^{-1}\left|\Delta \Sigma_{2}\right|_{l}<\infty
$$

where $l$ is the running index that consecutively numbers the curvature transitions in $\mathcal{A}_{\mathcal{T}} ;\left|\left(\Delta \Sigma_{2}\right)\right|_{l}$ then denotes the sum of the absolute changes in $\Sigma_{2}$ occurring for the frame transitions between the $(l-1)$ th and the $l$ th curvature transition. The series (H.8) is proved to converge in Appendix F; hence (H.7) converges. Finiteness of the sum (H.7) entails that $\bar{n}_{1}$ converges to a constant value (which depends on the spatial variables), i.e., $\bar{n}_{1}$ exhibits asymptotic freezing.

The arguments used to establish asymptotic freezing of $\bar{n}_{2}, \bar{n}_{3}$ are analogous. Instead of $E_{1} /\left(E_{2} R_{3}\right)$, one can use the quantity $E_{2} /\left(E_{3} R_{1}\right)$, which again exhibits a behavior of the type (H.5). In addition, the already established asymptotic constancy of $\bar{n}_{1}$ can be employed in the equation for $\bar{n}_{2}$.

Remark. Asymptotic freezing does not depend on the assumption that the solution converges to a attractor sequence (although this leads to simplifications in the computations). Analogously, one can show asymptotic freezing in the case that the asymptotic sequence converges to an arbitrary sequence of transitions on the billiard subset $\mathcal{B}$ (including double transitions). 
To establish asymptotic freezing of $\mathcal{P}_{i}$, we employ equation (H.4) and the analogous equations for the quantities $E_{1} /\left(E_{3} R_{2}\right)$ and $E_{2} /\left(E_{3} R_{1}\right)$ : obviously, for the inverse quantities $E_{2} R_{3} / E_{1}, E_{3} R_{2} / E_{1}, E_{3} R_{1} / E_{2}$, the evolution equations are of the form

$$
\partial_{\tau} A=2\left(1-\Sigma^{2}\right) A
$$

Furthermore, we note that the evolution equation for the quantity $\Lambda=2 H \sqrt{g}$ is

$$
\partial_{\tau} \Lambda=-2\left(1-\Sigma^{2}\right) \Lambda
$$

see Appendix A. From these equations it is immediate that

$$
\partial_{\tau} \mathcal{P}_{i}=0
$$

where we have used that $\bar{n}_{3}$ converges to a constant in the equation for $\mathcal{P}_{1}$. It follows that $\mathcal{P}_{i}$ are constant in the asymptotic regime so that asymptotic freezing holds trivially.

Remark. Based on the results of Appendix A, we are able to compute the following ratios:

$$
\begin{aligned}
\frac{R_{1} R_{3} \Lambda}{R_{2}} & =\frac{\mathcal{P}_{3}\left(n_{3} \mathcal{P}_{2}+\mathcal{P}_{1}\right)}{\mathcal{P}_{2}}, \quad \frac{N_{1} R_{3}^{2}}{N_{2} \Lambda^{2}}=\frac{\tilde{N}_{1}\left(n_{3} \mathcal{P}_{2}+\mathcal{P}_{1}\right)^{2}}{\tilde{N}_{2}} \\
\frac{E_{1} \Lambda}{E_{2} R_{3}} & =-\frac{1}{n_{3} \mathcal{P}_{2}+\mathcal{P}_{1}},
\end{aligned}
$$

where r.h.s., and hence also the l.h.s., are asymptotic constants of the motion. Since the quantity $\Lambda$ satisfies (A.19), it behaves like the auxiliary quantity $A$ (or like $B$ in the generic case); we thus find - a posteriori the asymptotic behavior of $R_{2}$ and $N_{2}$, in consistency with the results of Section 10.

\section{H.2 Asymptotic freezing of $\pi_{\lambda}$}

The momentum $\pi_{\lambda}$ conjugate to the variable $\lambda$ is given by

$$
\pi_{\lambda}=\Lambda \log \left[\left(e_{1}\right)^{\left(2-\Sigma_{1}\right)}\left(e_{2}\right)^{\left(2-\Sigma_{2}\right)}\left(e_{3}\right)^{\left(2-\Sigma_{3}\right)}\right],
$$

see Appendix A; recall that $e_{\alpha}$ is defined by $e_{\alpha}=H E_{\alpha}$ and that $\Lambda=2 H \sqrt{g}$. The derivative of the variables $e_{\alpha}$ reads

$$
\partial_{\tau} e_{\alpha}=\left(1+\Sigma_{\alpha}\right) e_{\alpha}
$$


cf. (A.18) in Appendix A. Using these formulas and equations (11.1a) to (11.1c), and (11.1g) for $\partial_{\tau} \Sigma_{\alpha}$, and (H.9) for $\partial_{\tau} \Lambda$, we find that the derivative of $\pi_{\lambda}$ is given by

$$
\Lambda^{-1} \partial_{\tau} \pi_{\lambda}=\log \left[\left(e_{1}\right)^{X_{1}}\left(e_{2}\right)^{X_{2}}\left(e_{3}\right)^{X_{3}}\right]+6\left(1-\Sigma^{2}\right)+2 R_{1}^{2}+2 R_{3}^{2},
$$

where

$$
X_{1}=-12\left(1-\Sigma^{2}\right)+2 R_{3}^{2}, \quad X_{2}=2\left(R_{1}^{2}-R_{3}^{2}\right), \quad X_{3}=-2 R_{1}^{2} .
$$

In order to establish asymptotic freezing of $\pi_{\lambda}$, we consider a sequence of transitions that is approximated by an attractor sequence $\mathcal{A}_{\mathcal{T}}$ in the asymptotic regime $\tau \rightarrow \infty$ and investigate (H.11) along this sequence of $\mathcal{T}_{N_{1}}, \mathcal{T}_{R_{1}}$, and $\mathcal{T}_{R_{3}}$ transitions. We begin by analyzing the behavior of certain functions in terms of the growth function $G$; we first note that

$$
\Lambda=2 H \sqrt{g}=\Lambda_{0} G^{-1}, \quad \text { so that } \Lambda_{i}=\Lambda_{0} G_{l(i)}^{-1} .
$$

From (11.1) and (H.10), we obtain the following equations:

$$
\begin{aligned}
& \partial_{\tau}\left[\left(1-\Sigma^{2}\right) e_{1}^{4}\right]=4\left(1-\Sigma^{2}\right)\left[\left(1-\Sigma^{2}\right) e_{1}^{4}\right] \\
& \partial_{\tau}\left[R_{1} \frac{e_{3}}{e_{2}}\right]=2\left(1-\Sigma^{2}\right)\left[R_{1} \frac{e_{3}}{e_{2}}\right], \quad \partial_{\tau}\left[R_{3} \frac{e_{2}}{e_{1}}\right]=2\left(1-\Sigma^{2}\right)\left[R_{3} \frac{e_{2}}{e_{1}}\right]
\end{aligned}
$$

hence the relations

$$
\left[\left(1-\Sigma^{2}\right) e_{1}^{4}\right] \propto G^{2}, \quad\left[R_{1} \frac{e_{3}}{e_{2}}\right] \propto G, \quad\left[R_{3} \frac{e_{2}}{e_{1}}\right] \propto G
$$

describe the behavior of these functions along $\mathcal{A}_{\mathcal{T}}$ in terms of growth function $G$. On the basis of these formulas, equation (H.11) simplifies. For a $\mathcal{T}_{R_{3}}$ frame transition, (H.11) reduces to

$$
\partial_{\tau} \pi_{\lambda}=\Lambda\left[2 R_{3}^{2} \log \frac{e_{1}}{e_{2}}+2 R_{3}^{2}\right]=2 \Lambda_{0} G^{-1} R_{3}^{2}\left[-\log G+\log R_{3}+\text { const }\right] .
$$

The fact that growth function $G$ is constant along the $\mathcal{T}_{R_{3}}$ transition makes it possible to integrate (H.13). Since $\partial_{\tau} \Sigma_{2}=2 R_{3}^{2}$, we obtain that

$$
2 \int R_{3}^{2} d \tau=\Delta \Sigma_{2}, \quad \text { and } \quad 2 \int R_{3}^{2} \log R_{3} d \tau=\Delta \Sigma_{2}\left(\log \Delta \Sigma_{2}-1\right),
$$


where we have used that $R_{3}^{2}=\left[\Sigma_{2}-\left(\Sigma_{2}\right)_{-}\right]\left[\left(\Sigma_{2}\right)_{+}-\Sigma_{2}\right]$ for the second integral; as usual, $\Delta \Sigma_{2}=\left(\Sigma_{2}\right)_{+}-\left(\Sigma_{2}\right)_{-}$, where $\left(\Sigma_{2}\right)_{ \pm}$denotes the values of $\Sigma_{2}$ at the endpoints of the transition. Using (H.14) we find that

$$
\Delta \pi_{\lambda}=\Lambda_{0} G^{-1} \Delta \Sigma_{2}\left[-\log G+\log \Delta \Sigma_{2}+\text { const }\right],
$$

or, in index notation, where we assume that $\mathcal{T}_{i}$ is the $\mathcal{T}_{R_{3}}$ transition under consideration:

$$
\left(\Delta \pi_{\lambda}\right)_{i}=\Lambda_{0} G_{l(i)}^{-1}\left(\Delta \Sigma_{2}\right)_{i}\left[-\log G_{l(i)}+\log \left(\Delta \Sigma_{2}\right)_{i}+\text { const }\right]
$$

here, the constant is independent of $i$. If $\mathcal{T}_{i}$ is a $\mathcal{T}_{R_{1}}$ transition instead of a $\mathcal{T}_{R_{3}}$, the result is analogous.

In the case of a $\mathcal{T}_{N_{1}}$ curvature transition, (H.11) reduces to

$$
\begin{aligned}
\partial_{\tau} \pi_{\lambda} & =6 \Lambda\left(1-\Sigma^{2}\right)\left[-2 \log e_{1}+1\right] \\
& =6 \Lambda_{0} G^{-1}\left(1-\Sigma^{2}\right)\left[-\log G+\frac{1}{2} \log \left(1-\Sigma^{2}\right)+\text { const }\right]
\end{aligned}
$$

The integral of $2\left(1-\Sigma^{2}\right)$ yields the logarithm of the growth factor $g=$ $\zeta_{+} / \zeta_{-}$associated with the transition $\mathcal{T}_{N_{1}}$, i.e.,

$$
2 \int\left(1-\Sigma^{2}\right) d \tau=\int \frac{d \zeta}{\zeta}=\log \frac{\zeta_{+}}{\zeta_{-}}=\log g
$$

The integral of $2\left(1-\Sigma^{2}\right) \log \left(1-\Sigma^{2}\right)$ is more involved; using (6.3b) we find

$$
\begin{aligned}
2 \int\left(1-\Sigma^{2}\right) \log \left(1-\Sigma^{2}\right) d \tau= & \left(\log \frac{3}{\zeta_{-} \zeta_{+}}\right) \log g \\
& +\int_{\zeta_{-}}^{\zeta_{+}} \frac{1}{\zeta} \log \left[\left(\zeta-\zeta_{-}\right)\left(\zeta_{+}-\zeta\right)\right] d \zeta
\end{aligned}
$$

If the Kasner parameter $u\left(=u_{-}\right)$of the $\mathcal{T}_{N_{1}}$ transition is large (i.e., if $\mathcal{T}_{N_{1}}$ belongs to a small curvature phase), then (H.17) simplifies by using the approximations

$$
g \approx 1+\frac{2}{u}, \quad \zeta_{+}-\zeta_{-} \approx \frac{6}{u^{3}}
$$


in particular

$$
\int_{\zeta_{-}}^{\zeta_{+}} \frac{1}{\zeta} \log \left[\left(\zeta-\zeta_{-}\right)\left(\zeta_{+}-\zeta\right)\right] d \zeta \approx-\frac{12}{u}\left[\log u-\frac{\log 6-1}{3}\right]
$$

so that

$$
2 \int\left(1-\Sigma^{2}\right) \log \left(1-\Sigma^{2}\right) d \tau \approx \frac{2}{u}[-2 \log u+\log 12-2]
$$

for $u \gg 1$. Since $G$ is not constant along the $\mathcal{T}_{N_{1}}$ orbit, equation (H.16) cannot be integrated explicitly. However, by making the estimate $G \geq G_{-}$, where $G_{-}$is the initial value of the growth function, we obtain

$$
\left|\Delta \pi_{\lambda}\right| \leq 3 \Lambda_{0} G_{-}^{-1}\left[\log G_{-} \log g+\text { const } \log g+\left|\int\left(1-\Sigma^{2}\right) \log \left(1-\Sigma^{2}\right) d \tau\right|\right]
$$

which reduces to

$$
\left|\Delta \pi_{\lambda}\right| \leq 6 \Lambda_{0} G_{-}^{-1} \frac{1}{u}\left[\log G_{-}+\log u+\text { const }\right]
$$

in the case $u \gg 1$. In index notation, when we assume that the $\mathcal{T}_{N_{1}}$ transition under consideration is the $i$ th transition of the sequence, i.e., $\mathcal{T}_{i}=\mathcal{T}_{N_{1}}$, we have

$$
\left|\Delta \pi_{\lambda}\right|_{i} \leq 6 \Lambda_{0} G_{l(i)}^{-1} \frac{1}{u_{l(i)}}\left[\log G_{l(i)}+\log u_{l(i)}+\text { const }\right]
$$

where the estimate holds in a small curvature phase (with sufficiently large $\left.u_{l}\right)$.

To establish asymptotic freezing of $\pi_{\lambda}$, we must show that the summation of all $\left(\Delta \pi_{\lambda}\right)_{i}$ is finite (where the sum is over all transitions of $\mathcal{A}_{\mathcal{T}}$ ), i.e.,

$$
\sum_{i}\left|\Delta \pi_{\lambda}\right|_{i}<\infty
$$

Finiteness of this sum entails that $\pi_{\lambda}$ converges to a constant value (depending on the spatial variables), i.e., $\pi_{\lambda}$ exhibits asymptotic freezing.

We merely state the main arguments of the proof and omit the computational details: in analogy to the considerations of Appendix F, we may focus on the behavior of (H.19) in small curvature phases (i.e., the alternating phases of $\mathcal{T}_{R_{3}}$ and $\mathcal{T}_{N_{1}}$ transitions in the neighborhood of $\mathrm{T}_{3}$ ), since in large 
curvature phases, the treatment of the sum is simple. Since $\left|\Delta \Sigma_{2}\right| \sim u^{-1}$ when $u \gg 1$, see (6.2), equations (H.15) and (H.18) are of the same type and lead to sums of the type

$$
\sum_{l} G_{l}^{-1} \log G_{l} \frac{1}{u_{l}} \text { and } \sum_{l} G_{l}^{-1} \frac{\log u_{l}}{u_{l}}
$$

where $l$ is the running index that consecutively numbers the curvature transitions in $\mathcal{A}_{\mathcal{T}}$. Finiteness of (H.20) is proved in Appendix F, hence finiteness of (H.19) ensues and we obtain asymptotic freezing of $\pi_{\lambda}$.

\section{AVTD singularities and the dynamical systems approach}

In this appendix, we discuss the connection between work on AVTD singularities and the dynamical systems approach. There exist no rigorous results for inhomogeneous models with oscillatory singularities, but there are some for inhomogeneous models with so-called AVTD singularities. In this appendix, we address how work in this latter field relates to the present approach.

The concept of velocity dominated singularities was first introduced by Eardley et al. [51]. Isenberg, Moncrief and co-workers later used the approach of Eardley et al. to obtain rigorous results, see [42] and references in [16]. Let us here follow Isenberg and Moncrief [42]. They use the standard initial value problem as their starting point, the spatial metric and the extrinsic curvature being the dependent variables. They then consider the system of differential equations one obtains if one drop all spatial derivatives, except in the Codazzi constraints, and sets the three-curvature to zero. By doing so, they obtain a VTD ODE system of evolutionary equations constrained algebraically by the Gauss constraint; the solution of this system can subsequently be inserted into the Codazzi constraints yielding a VTD solution. A spacetime is said to be asymptotically velocity term dominated (AVTD) if it is a solution to Einstein's equations such that it, w.r.t. some appropriate norm, asymptotically approach a solution to the VTD equations, see $[42$, p. 87,88$]$ for details. If furthermore the asymptotic limit yields a singularity, this singularity is said to be AVTD.

If one uses a conformal Hubble-normalized orthonormal frame, then the equations for the VTD ODE system are the Bianchi type I evolution equations and the Gauss constraint on the silent boundary - and the equations for $E_{\alpha}{ }^{i}$ and the Hubble variable $H$, with all spatial frame derivatives dropped and all variables set to zero except for $\Sigma_{\alpha \beta}$ (and possible matter variables), 
i.e., the lowest order perturbations of the evolution equations for $E_{\alpha}{ }^{i}$ and $H$ w.r.t. the silent Bianchi type I subset; this therefore yields the generalized Bianchi type I solutions, which in the vacuum case are the generalized Kasner solutions. The VTD treatment of the Codazzi constraints corresponds to inserting these solutions into the lowest non-zero perturbation of the constraints w.r.t. the silent Bianchi type I subset (i.e., one sets all variables to zero except for $E_{\alpha}{ }^{i}, \Sigma_{\alpha \beta}$, and possible matter variables, in the constraints).

In the vacuum case, AVTD singularities occur for special spacetimes, notably ones with symmetries, as discussed in Section 13. In cases with one or two commuting spacelike Killing vectors, this naturally leads to an Iwasawa frame representation, and hence to a fairly simple correspondence between the present Iwasawa-based conformally Hubble-normalized variables and variables that have been used in previous work in this area (thus the AVTD starting point is obtained by plugging in the Kasner circle $\mathrm{K}^{\bigcirc}$ into the lowest order approximation for $E_{\alpha}{ }^{i}$ and $H$, as done in Appendix B).

Arguably the most impressive result about AVTD singularities was obtained by Andersson and Rendall [17] for massless scalar fields and stiff perfect fluids. In this case, the Bianchi type I equations on the silent boundary yields the generalized Jacobs solutions, and it turns out that an open subset of these solutions are stable and hence attract an open set of solutions. It should, however, be pointed out that the approach of Andersson and Rendall is naturally related to a Fermi propagated frame rather than an Iwasawa frame. Stiff perfect fluid spacetimes has also been investigated in terms of the UEWE dynamical systems approach by Coley and $\operatorname{Lim}[52]$.

We note that within the present framework AVTD singularities are more appropriately geometrically described as singularities that are associated with (Hubble) asymptotic (Hubble) conformal spatial flatness.

To study when singularities are not AVTD, Moncrief and coworkers have introduced the "Method of Consistent Potentials" [15, 43], and references in [16]. In this case, one inserts the VTD solutions (the generalized Kasner solutions in the vacuum case) into the full equations (or rather, into the Hamiltonian for the full equations); if all terms are decaying the spacetime is AVTD, if not it is conjectured to have local Mixmaster dynamics. We note that this corresponds to the linear perturbation of the Kasner circle $\mathrm{K}^{\bigcirc}$ in the present context, as can be seen by inserting the generalized Kasner solutions into the relations given in Appendix A and comparing with the linearized $\mathrm{K}^{\bigcirc}$ result. Here, however, this result is seen in the context of the full conformally normalized state space picture. 
We note that the Hamiltonian approach using the "dominant" Hamiltonian $\mathcal{H}$ suggests a direct generalization of the VTD/AVTD approach, or equivalently, the billiard subset and its lowest order perturbation into the physical state space (with the ODE solution, yielding a lowest order perturbation, inserted into the constraints), see Section 12. An advantage of the present approach is that it naturally splits the problem into two parts and hence offers a possibility of achieving more modest goals than cracking the whole problem: it may be possible to prove some statements that are connected with the silent boundary only — it may not be necessary to also prove things about perturbations thereof.

\section{References}

[1] R. Penrose and S.W. Hawking. The singularities of gravitational collapse and cosmology, Proc. Roy. Soc. Lond. A 314 (1970), 529.

[2] E.M. Lifshitz and I.M. Khalatnikov, Investigations in relativistic cosmology, Adv. Phys. 12 (1963), 185.

[3] V.A. Belinskiı̌, I.M. Khalatnikov and E.M. Lifshitz, Oscillatory approach to a singular point in the relativistic cosmology, Adv. Phys. 19 (1970), 525.

[4] - A general solution of the Einstein equations with a time singularity, Adv. Phys. 31 (1982), 639.

[5] I.M. Khalatnikov, E.M. Lifshitz, K.M. Khanin, L.N. Shur and Ya.G. Sinai, On the stochasticity in relativistic cosmology, J. Stat. Phys. 38 (1985), 97.

[6] J.D. Barrow, Chaotic behaviour in general relativity, Phys. Rep. 85 (1982), 1.

[7] D.F. Chernoff and J.D. Barrow, Chaos in the Mixmaster Universe, Phys. Rev. Lett. 50 (1983), 134.

[8] C.W. Misner, Quantum cosmology I, Phys. Rev. 186 (1969), 1319.

[9] C.W. Misner, K.S. Thorne and J.A. Wheeler, Gravitation, W. H. Freeman and Company, San Francisco, 1973.

[10] D.M. Chitré, Ph.D. thesis, University of Maryland, 1972.

[11] R.T. Jantzen, Spatially homogeneous dynamics: a unified picture, arXiv:gr-qc/0102035.

[12] J. Wainwright and G.F.R. Ellis, Dynamical systems in cosmology. Cambridge University Press, Cambridge, 1997.

[13] T. Damour, M. Henneaux and H. Nicolai, Cosmological billiards, Class. Quantum Grav. 20 (2003), R145. 
[14] T. Damour and H. Nicolai. Higher order $M$ theory corrections and the Kac-Moody algebra E10, Class. Quantum Grav. 22 (2005), 2849.

[15] B.K. Berger, D. Garfinkle, J. Isenberg, V. Moncrief and M. Weaver, The singularity in generic gravitational collapse is spacelike, local, and oscillatory, Mod. Phys. Lett. A 13 (1998), 1565.

[16] B.K. Berger, Numerical approaches to spacetime singularities, Living Rev. Relativ. 5 (2002), 6.

[17] L. Andersson and A.D. Rendall, Quiescent cosmological singularities, Commun. Math. Phys. 218 (2001), 479.

[18] H. Ringström, Curvature blow up in Bianchi VIII and IX vacuum spacetimes, Class. Quantum Grav. 17 (2000), 713-731.

[19] - The Bianchi IX attractor, Ann. Henri Poincaré 2 (2001), 405.

[20] C. Uggla, H. van Elst, J. Wainwright and G.F.R. Ellis, The past attractor in inhomogeneous cosmology, Phys. Rev. D 68 (2003), 103502.

[21] D. Garfinkle, Numerical simulations of generic singularities, Phys. Rev. Lett. 93 (2004), 161101.

[22] L. Andersson, H. van Elst, W.C. Lim and C. Uggla, Asymptotic silence of generic singularities, Phys. Rev. Lett. 94 (2005), 051101.

[23] W.C. Lim, C. Uggla and J. Wainwright, Asymptotic silence-breaking singularities, Class. Quantum Grav. 23 (2006), 2607.

[24] N. Röhr and C. Uggla, Conformal regularization of Einstein's field equations, Class. Quantum Grav. 22 (2005), 3775.

[25] L.P. Grishchuk, A.G. Doroshkevich and V.N. Lukash, The model of "mixmaster universe" with arbitrarily moving matter, Zh. Eksp. Teor. Fiz. 61 (1971), 3 [Sov. Phys. JETP 34 (1972), 1].

[26] W.C. Lim, H. van Elst, C. Uggla and J. Wainwright, Asymptotic isotropization in inhomogeneous cosmology, Phys. Rev. D 69 (2004), 103507.

[27] B.S. DeWitt. Quantum gravity I, Phys. Rev. 160 (1967), 1113.

[28] D.M. Eardley, Self-similar spacetimes: geometry and dynamics, Comm. Math. Phys. 37 (1974), 287.

[29] L. Andersson, H. van Elst and C. Uggla, Gowdy phenomenology in scale-invariant variables, Class. Quant. Grav. 21 (2004), S29.

[30] S. Matarrese, O. Pantano and D. Saez, General relativistic dynamics of irrotational dust: Cosmological implications, Phys. Rev. Lett. 72 (1994), 320.

[31] M. Bruni, S. Matarrese and O. Pantano, Dynamics of silent universes, Astrophys. J. 445 (1995), 958. 
[32] H. van Elst, C. Uggla, W. Lesame, G.F.R. Ellis and R. Maartens, Integrability of irrotational silent cosmological models, Class. Quant. Grav. 14 (1997), 1151.

[33] L. Wylleman and N. Van den Bergh, Complete classification of purely magnetic, non-rotating and non-accelerating perfect fluids, arXiv:grqc/0604025.

[34] D. Christodoulou and S. Klainerman, The global nonlinear stability of the Minkowski space, Princeton University Press, Princeton, New Jersey, 1993.

[35] M. Dafermos and I. Rodnianski, A proof of Price's law for the collapse of a self-gravitating scalar field, Invent. Math. 162 (2005), 381.

[36] C.G. Hewitt, J.T. Horwood and J. Wainwright, Asymptotic dynamics of the exceptional Bianchi cosmologies, Class. Quant. Grav. 20 (2003), 1743.

[37] P.K-H. Ma, A dynamical systems approach to the oscillatory singularity in cosmology, M. Math. thesis, University of Waterloo, unpublished, 1988.

[38] Y. Choquet-Bruhat, J. Isenberg and V. Moncrief, Topologically general U(1) symmetric Einstein spacetimes with AVTD behavior, Nuovo Cim. 119B (2004), 625.

[39] Y. Choquet-Bruhat and J. Isenberg, Half polarized U(1) symmetric vacuum spacetimes with AVTD behavior, J. Geom. Phys. 56 (2006), 1199.

[40] J. Wainwright, A classification scheme for non-rotating inhomogeneous cosmologies, J. Phys. A Math. Gen. 12 (1979), 2015.

[41] H. van Elst, C. Uggla and J. Wainwright, Dynamical systems approach to G2 cosmology, Class. Quant. Grav. 19 (2002), 51.

[42] J.A. Isenberg and V. Moncrief, Asymptotic behavior of the gravitational field and the nature of singularities in the Gowdy spacetimes, Ann. Phys. (N.Y.) 199 (1990), 84.

[43] B. Grubišić and V. Moncrief, Asymptotic behavior of the $T^{3} \times R$ Gowdy space-times, Phys. Rev. D. 47 (1993), 2371.

[44] R. Benini and G. Montani. Frame independence of the inhomogeneous mixmaster chaos via Misner-Chitré-like variables, Phys. Rev. D. 70 (2004), 103527-1.

[45] W.C. Lim, The dynamics of inhomogeneous cosmologies, Ph.D. thesis, University of Waterloo, 2004, arXiv:gr-qc/0410126.

[46] J.M. Heinzle and C. Uggla, Dynamics of the spatially homogeneous Bianchi type I Einstein-Vlasov equations, Class. Quant. Grav. 23 (2006), 3463. 
[47] A. Ashtekar, R.S. Tate and C. Uggla, Minisuperspaces: observables and quantization, Int. J. Mod. Phys. D 2 (1993), 15.

[48] - Minisuperspaces: symmetries and quantization, in 'Misner Festschrift', eds. B.L. Hu et al., Cambridge University Press, 1993, arXiv:gr-qc/9302026.

[49] M. Fjällborg, J.M. Heinzle and C. Uggla, Self-gravitating stationary spherically symmetric systems in relativistic galactic dynamics, 2006, arXiv:gr-qc/0609074.

[50] H. Ringström, Future asymptotic expansions of Bianchi VIII vacuum metrics, Class. Quant. Grav. 20 (2003), 1943.

[51] D.M. Eardley, E. Liang and R. Sachs, Velocity-dominated singularities in irrotational dust cosmologies, J. Math. Phys. 13 (1972), 99.

[52] A.A. Coley and W.C. Lim, Asymptotic analysis of spatially inhomogeneous stiff and ultra-stiff cosmologies, Class. Quant. Grav. 22 (2005), 3073 . 
